\title{
DETERMINATION OF THE DYNAMIC GAIN FUNCTION OF CORTICAL INTERNEURONS WITH DISTINCT ELECTRICAL TYPES
}

\author{
Dissertation \\ in partial fulfillment of the requirements \\ for the degree \\ "Doctor rerum naturalium" \\ in the Neuroscience Program \\ at the Georg-August-Universität Göttingen, \\ Faculty of Biology
}

Submitted by

Ricardo Martins Merino

born in

Ribeirão Preto, Brazil

Göttingen, 2016 



\section{Members of the thesis committee:}

- Prof. Dr. Fred Wolf (Supervisor, referee) Department of Nonlinear Dynamics, Max Planck Institute for Dynamics and Self-Organization

- Prof. Dr. Walter Stühmer (2nd referee) Department of Molecular Biology of Neuronal Signals Max Planck Institute of Experimental Medicine

- Dr. Andreas Neef (Co-Supervisor) Department of Nonlinear Dynamics Max Planck Institute for Dynamics and Self-Organization

Other members of the examination board:

- Prof. Dr. Jochen Staiger Institute for Neuroanatomy University Medical Center, Georg-August-University

- Dr. Oliver Schlüter European Neuroscience Institute

- Prof. Dr. Siegrid Löwel Department of Systems Neurosciences Georg-August-University

Date of oral examination: December 21st, 2016 



\section{STATEMENT OF ORIGINALITY}

I hereby declare that I prepared the PhD Thesis entitled "DETERMINATION OF THE DYNAMIC GAIN FUNCTION OF CORTICAL INTERNEURONS WITH DISTINCT ELECTRICAL TYPES" on my own and with no other sources and aids than cited.

Göttingen, November 7th, 2016

Ricardo Martins Merino 



\section{TABLE OF CONTENTS}

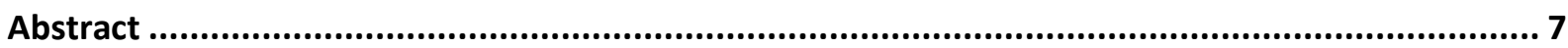

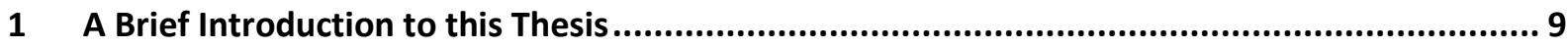

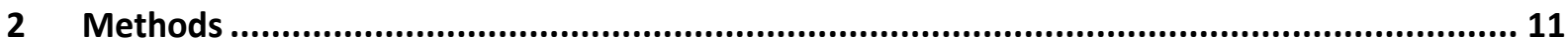

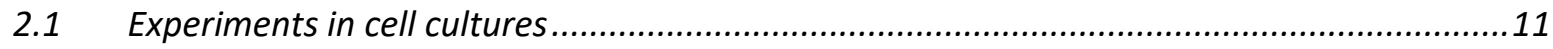

2.1.1 Cloning of multiple channelrhodopsin variants .........................................................11

2.1.2 HEK cell cultures and transient transfection ...............................................................

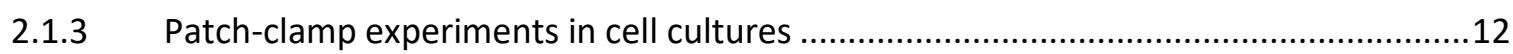

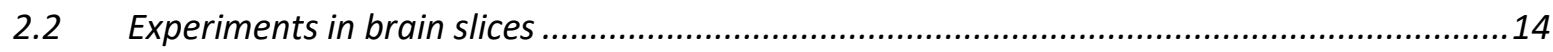

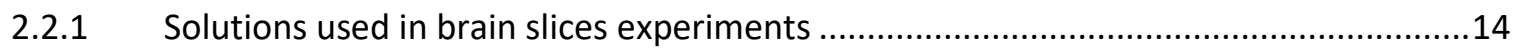

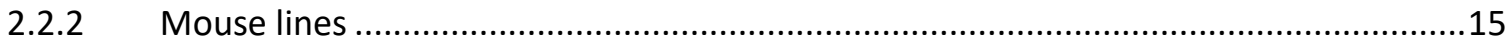

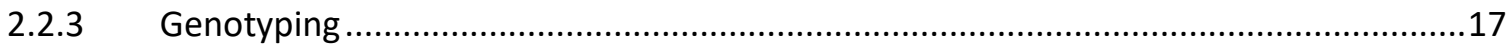

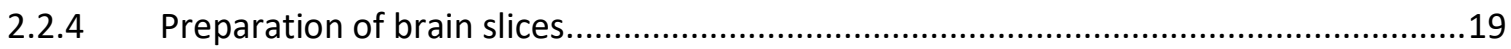

2.2.5 Patch-clamp experiments in brain slices...................................................................19

3 Chapter 1: Optogenetic Tools for Characterizing Neuronal Transfer Functions....................... 25

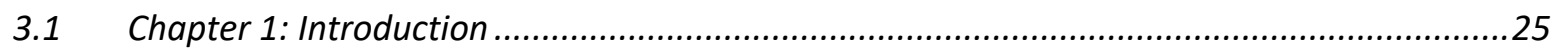

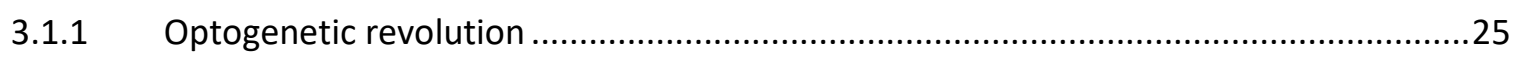

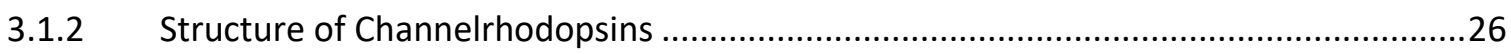

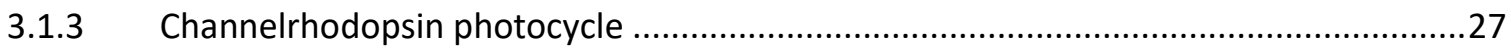

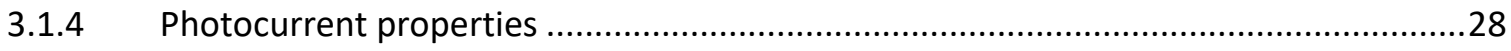

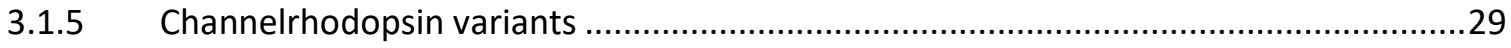

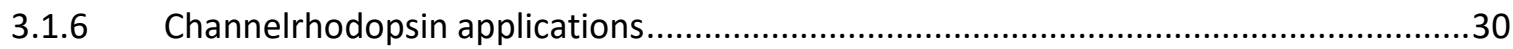

3.1.7 Continuous Dynamic Photostimulation - CoDyPS .......................................................

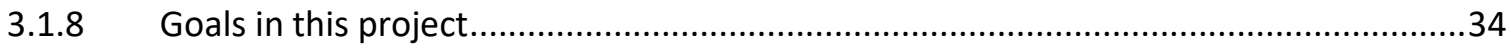

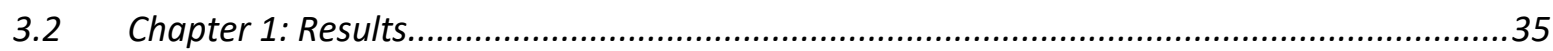

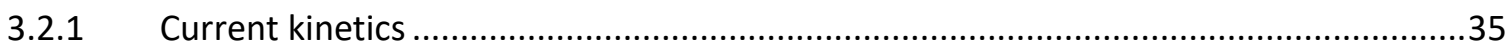

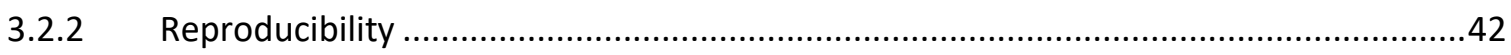

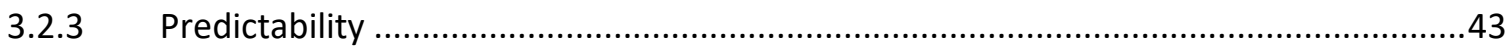

3.2.4 Tests in neurons: lack of neuronal expression ...........................................................4

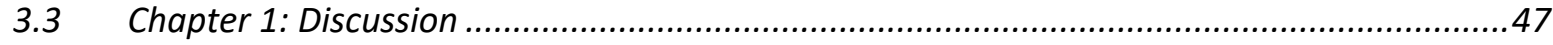


3.3.1 Advantages of using optogenetics for determining neuronal transfer functions..........47

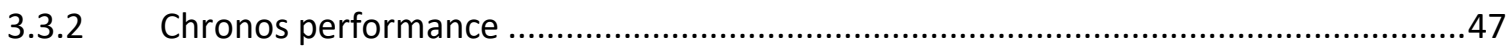

4 Chapter 2: The gain function in electrically defined interneuronal populations .................... 51

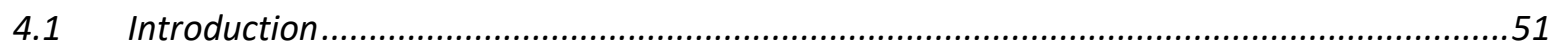

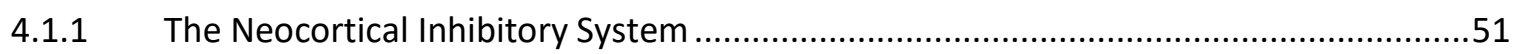

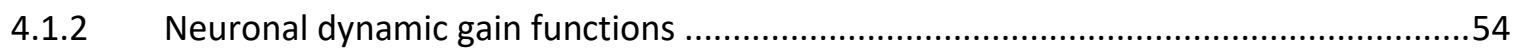

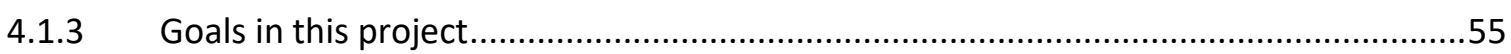

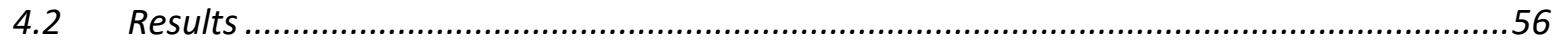

4.2.1 Determination of the electrical type of interneurons ...................................................56

4.2.2 Different electrical types encode information differently ...........................................60

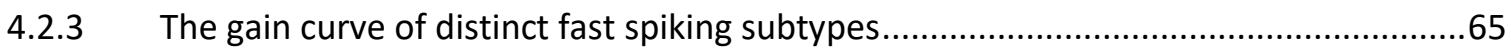

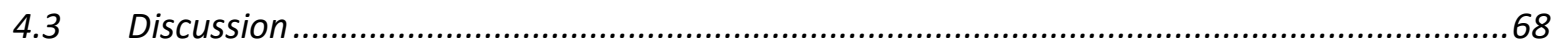

4.3.1 What is the relationship between the electrical types here described and

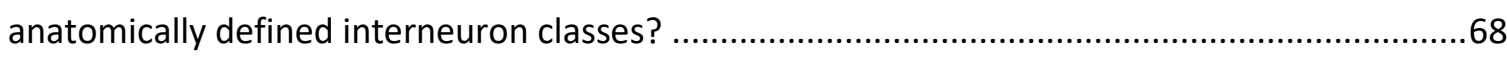

4.3.2 What could be the main determinants of the different gain curves seen in different

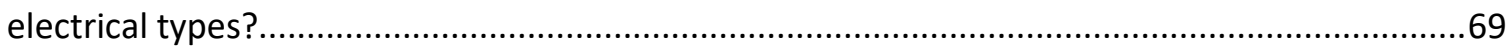

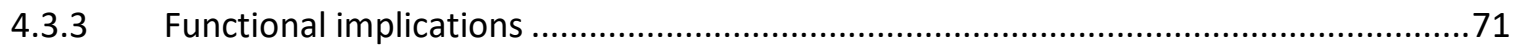

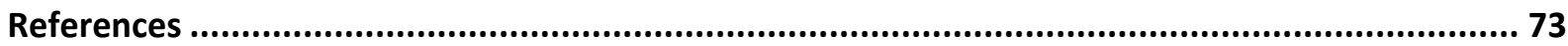

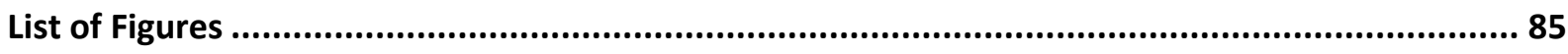

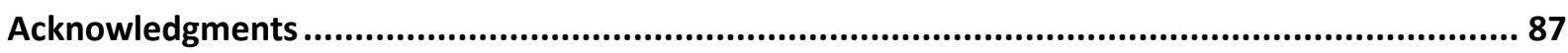

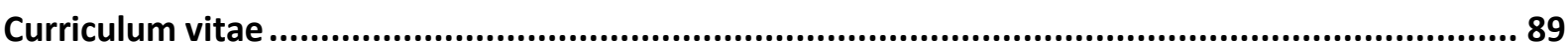




\begin{abstract}
In the living brain, individual neurons are constantly bombarded by thousands of synaptic inputs, which results in a fluctuating membrane potential. Neurons under such conditions are said to operate in a "fluctuation-driven regime", in which stochastic incursions of the membrane potential to suprathreshold values result in the emission of action potentials. The process of transforming inputs, i.e., the fluctuating membrane potential, into an output, the spikes, is called "information encoding". The dynamic gain function is a way to identify how this encoding takes place, by identifying the relationship between input frequencies and neuronal output. In this thesis, I sought to advance our understanding of how nerve cells encode information by means of two different approaches. In the first approach, a technical one, I characterized optogenetic tools that can be used to facilitate the traditionally laborious and time consuming determination of the neuronal gain function. Among the fast light sensitive channels available to date, chronos was the most promising. However, while it fulfilled all the basic requirements for a noninvasive fluctuating light stimulation, issues with respect to its level of expression in neurons hinder its applicability. In the second front, I used electrophysiological tools to effectively characterize the dynamic gain function of distinct electrical types of interneurons. I showed that fast spiking and adapting interneurons exhibit different frequency preferences, and that the correlation time of the noise input differently affects the gain curves of these cells. In the fast noise regime, adapting neurons exhibited a low-pass filter-like behavior, with peak gain in the theta range (1-10 Hz), while fast spiking cells showed a band-pass filter behavior with strong resonance in the $100-200 \mathrm{~Hz}$ band. Interestingly, in the slow noise regime, while fast spiking gain behavior qualitatively did not change, adapting interneurons exhibited a band-pass-like behavior, with peak at $100 \mathrm{~Hz}$. In order to further characterize the gain of fast spiking neurons, these cells were subdivided into three categories: continuous, delayed, and stuttering. The gain calculation of each of these three subtypes showed that, while in the fast regime their responses were considerably similar, in the slow regime they exhibited distinct resonance peaks, with a considerable variation of the gain at the peak. To the best of my knowledge, this is the first time that the gain of inhibitory interneurons is characterized in the noise-driven regime.
\end{abstract}





\section{A Brief Introduction to this Thesis}

I deem the understanding of neural systems, and particularly that of the human brain, one of the last frontiers of the scientific endeavor. Given the complexity of even the simplest nervous systems, multiple approaches can, and must, be used in order to advance in this field. Neurons come in a multitude of different flavors, each with its own uniqueness. Over more than a century, efforts have been made in order understand how many neuronal types there are, what they look like, where they are and to whom they talk, and, likewise, whom they listen to. In a way, the work here described addressed this last point, but in a functional manner. My work was focused in determining the so-called frequency-response, or dynamic gain function, of subtypes of interneurons, and in how one can optimize the determination thereof.

In the living brain, neurons are constantly bombarded by thousands of synaptic inputs from their presynaptic partners. These inputs occur at different subdomains of the postsynaptic neuron, and can exhibit very distinct kinetic properties. As all these incoming signals travel down from dendrites and soma to the axon initial segment, where they will finally be interpreted by the spike initiation machinery, significant modification, or filtering, occur in the signals. The result of such process is a highly variable, fluctuating membrane potential, formed by the complex integration and filtering of distinct synaptic inputs by the cellular membrane. Neurons operating under such conditions are said to operate in a "fluctuation-driven regime", in which stochastic incursions of the potential to suprathreshold values result in the emission of action potentials. The process of transforming the input, the fluctuating membrane potential, onto an output, the spikes, is called "information encoding", and the dynamic gain function is a way to identify how this encoding takes place. Essentially, the method reveals to which input frequency bands certain cell types optimally respond to. In other words, by means of gain function determination, one can reveal hidden input features embedded in the noise background, features which offer valuable information about the very role of a certain neuronal population in terms of information encoding and its contribution to network related activity, such as network oscillations. In addition, such understandings also contribute immensely to the development of theoretical and computational models employed to understand these two aspects of brain function.

My work is divided in two fronts. In one, Chapter One (p. 25), I describe the work I did aiming at optimizing tools that facilitate the determination of neuronal gain functions. 
Considering that one needs to record thousands of action potentials in order to extract those features hidden in the noise stimulus, the present techniques (e.g., intracellular or patchclamp recordings) show a limitation in the time a neuron can be recorded due to their invasive nature. In addition, with present techniques hardly more than one cell at a time can be recorded simultaneously. If we had available a non-invasive method to, both, stimulate neurons as well as record their activity, one could significantly facilitate data acquisition. By parallelizing the procedure, for example by means of simultaneous extracellular multielectrodes recordings of multiples cells, the throughput of the procedure can potentially be extended by many orders of magnitude. The approach I used in order to try to advance in this front was optogenetics. In Chapter One I explain the requirements channelrhodopsins must have, if they are to be used for dynamic gain calculations. I show that one channelrhodopsin variant, called chronos, fulfills all of these requirements. However, further optimization of this channelrhodopsin in terms of neuronal expression capabilities is necessary, so one can take full advantage of it.

In the second front, Chapter Two (p. 51), I set out to investigate how different interneurons, as defined by their electrical types, encode information in a noise-driven regime paradigm. I show that, differently from pyramidal neurons, these interneurons are especially sensitive to high-frequency noise input components, exhibiting a band-pass like filter behavior, as opposed to the common low-pass filter behavior of pyramidal neurons. I also show that different electrical types operate differently depending on the noise statistics used; and that, for a particular type, a transition between low-frequency input preference, to high-frequency input preference, can be induced by different noise regimes.

The methods employed in each section are detailed in Section 2 (p. 11), and are basically separated in two. The first part, "Experiments in cell cultures", refers to the methods employed in Chapter 1, and the second, "Experiments in brain slices", concerns the techniques I used for determining the gain of interneurons (Chapter 2). 


\section{Methods}

\subsection{Experiments in cell cultures}

Experiments in cell cultures consisted of patch-clamp recordings of HEK cells transiently transfected with various channelrhodopsins. The goal of such experiments was to evaluate the performance of "fast variants" of channelrhodopsins aiming at identifying potential candidates suitable for applications demanding high speed. The tested channels were the following: channelrhodopsin-2 and CatCh (kindly donated by Prof. Dr. Ernst Bamberg; Kleinlogel et al., 2011; Nagel et al., 2003), ChETA, variant E123T/T159C (kindly donated by Prof. Peter Hegemann; Prigge et al., 2012), and chronos (kindly donated by Prof. Dr. Edward Boyden; Klapoetke et al., 2014).

\subsubsection{Cloning of multiple channelrhodopsin variants}

Donated plasmids were first amplified before transfection. For this, $50 \mu \mathrm{l}$ of DH5 $\alpha$ competent bacteria suspension were mixed with $1 \mu \mathrm{g}$ of plasmid DNA and incubated on ice for $20 \mathrm{~min}$, followed by a $2 \mathrm{~min}$ heat-shock at $42^{\circ} \mathrm{C}$. $500 \mu \mathrm{l}$ of lysogeny broth (LB) medium was added to the tube and cells were incubated shaking for $1 \mathrm{~h}$ at $37^{\circ} \mathrm{C}$. Approximately $50 \mu \mathrm{l}$ of cell suspension was then streaked onto an agar plate containing the proper selective antibiotic, and plate was kept overnight in an incubator at $37{ }^{\circ} \mathrm{C}$. After the overnight incubation, a single colony of the agar plate was chosen and with a sterile pipette tip inoculated into a liquid culture flask containing $100 \mathrm{ml}$ of LB medium with $0.1 \%(\mathrm{v} / \mathrm{v})$ of the proper antibiotic. Under vigorous shaking (approximately $300 \mathrm{rpm}$ ), cells were once more incubated overnight at $37^{\circ} \mathrm{C}$. Cells were then harvested by centrifugation at $6000 \mathrm{x} \mathrm{g}$ for 15 min at $4{ }^{\circ} \mathrm{C}$ and plasmids were purified with an endonuclease-free MidiPrep kit, following the manufacturer's instructions (Endofree NucleoBond Xtra Midi, Macherey-Nagel).

\subsubsection{HEK cell cultures and transient transfection}

HEK-293 cells were obtained from the German Collection of Microorganisms and Cell Cultures (DSMZ, ACC 305) and grown in Dulbecco's Modified Eagle Medium with nutrient mixture F12 (DMEM/F12, ThermoFisher) supplemented with $10 \%$ fetal calf serum (FCS; 
Biochrom $\mathrm{GmbH}$ ). Cells were kept in cell culture T75 flasks (Sarstedt) at $37{ }^{\circ} \mathrm{C}$ under humidified atmosphere containing 5 \% CO2 and were subcultured two times per week, when they reached 80-90\% confluence. For subculturing, medium was sucked out of the flask and cells were rinsed once with $10 \mathrm{ml}$ PBS, which was then discarded. Cells were then incubated in $1 \mathrm{ml}$ of Trypsin/EDTA (0.05\%, $0.02 \%, \mathrm{w} / \mathrm{v}$ in standard phosphate-buffered saline) at 37 ${ }^{\circ} \mathrm{C}$ for $3 \mathrm{~min}$. Trypsinization was stopped by adding $5 \mathrm{ml}$ of FCS-supplemented DMEM/F12 medium and cells were homogenized by pipetting. Cell suspension was transferred to a centrifuge tube and centrifuged for $2 \mathrm{~min}$ at $1200 \mathrm{rpm}$. Supernatant was discarded and cell pellet resuspended in $1 \mathrm{ml}$ of medium. Approximately $100 \mu \mathrm{l}$ of the cell suspension was added to a new flask with $10 \mathrm{ml}$ of fresh medium.

For the transient transfection (nucleofection; Lonza) of HEK cell with various channelrhodopsin plasmids, 3-5 $\mu \mathrm{g}$ of DNA and the Amaxa Cell Line Nucleofection Kit V (Lonza; program Q-01), were used following the manufacturer's instructions. After nucleofection, transfected cells were platted onto poly-L-lysine pre-coated $10 \mathrm{~mm}$ glass coverslips and incubated as described above.

\subsubsection{Patch-clamp experiments in cell cultures}

Approximately $20 \mathrm{~h}$ after plating, transfected HEK cells were patch-clamp recorded. Each coverslip at a time was transferred to the recording chamber of an inverted Axiovert 135 TV fluorescence microscope, equipped with a 40X/0.65 Achroplan objective. Cells were either recorded at room temperature or at approximately $35{ }^{\circ} \mathrm{C}$. External solution's composition was as following (in mM): $145 \mathrm{NaCl}, 3 \mathrm{KCl}, 1 \mathrm{MgCl}_{2}, 2 \mathrm{CaCl}_{2}, 10 \mathrm{HEPES}$, and 15 Glucose (osmolarity ranging from 310 to $315 \mathrm{mOsm}$ and $\mathrm{pH}=7.35$ ). When recorded at $35^{\circ} \mathrm{C}$, warmed external solution was constantly perfused through the recording chamber via an inline heater (HPT-2, Alasciences) controlled by a temperature controller (TC-10, NPI). Temperature was constantly measured via a thermistor placed within the recording chamber and connected to the temperature controller. Transfected cells were identified by the expression of fluorescent reporter proteins, and cell healthiness checked through transmitted-light. Whole-cell patch-clamp recordings in voltage-clamp mode were made with an EPC 10 USB amplifier (Heka). Membrane potential was clamped at $-60 \mathrm{mV}$, and current signals were low-pass filtered at $3 \mathrm{kHz}$ and digitized at $20 \mathrm{kHz}$. Patch-pipettes were pulled from PG10165-4 glass (World Precision Instruments). Electrode resistances were between 3$5 \mathrm{MOhm}$ when filled with the following internal solution (in mM): $110 \mathrm{NaCl}, 10 \mathrm{Na}_{4}$-EGTA, 4 $\mathrm{MgCl}_{2}, 10$ HEPES, and 10 Glucose ( $\mathrm{pH}=7.4$ and osmolarity ranging from 285 to $290 \mathrm{mOsm}$ ). Series resistance was always 60-90 \% compensated, with $100 \mu$ s feedback time. Immediately 
before touching the cell, the objective was replaced by a custom-made light-source screwed to the microscope's revolver. Light-elicited currents were analyzed offline in Matlab (Matlab 2011b/2014b, Mathworks), Igor (Igor Pro 6, Wavemetrics) and OriginPro 7 (OriginLab).

\subsubsection{1 $\quad$ Optogenetic stimulation}

For optogenetic stimulation I used a custom-built light source equipped with a blue light-emitting diode (LED, Luxeon Rebel color with Lambertian dome; Philips Lumileds). Light output was controlled via a custom-built LED controller fed with voltages from the patch-clamp amplifier. Each volt at the D/A board of the amplifier resulted in $1 \mathrm{~W}$ of light at the LED. Stimulation protocols consisted of (1) 1s-long light steps of different intensities $\left(0.08,0.14,0.19,0.23,0.27 \mathrm{~mW} / \mathrm{mm}^{2}\right)$, with a $11 \mathrm{~s}$-long dark period in between, (2) $10 \mathrm{~s}$-long light chirp linearly-increasing from 0.1 to $100 \mathrm{~Hz}$ and maximum amplitude of $0.27 \mathrm{~mW} / \mathrm{mm}^{2}$, and (3) fluctuating light stimuli created from a stochastic process based on an OrnsteinUhlenbeck process (Destexhe et al., 2001). Fluctuating voltage signals were created from Gaussian noise $\left(\bar{V}+\sigma(1+\kappa)^{0.5} \cdot(1-\kappa)^{-0.5} \cdot \xi_{i}\right)$, in which $\bar{V}$ is the average voltage, $\sigma$ is the voltage's standard deviation, $\kappa=\exp \left(-\Delta t / \tau_{-} \operatorname{corr}\right), \xi_{i}$ is a random number sequence, and $\Delta \mathrm{t}$ is the time step. The created noise was then passed through an RC-type low-pass filter with the desired time constant $\tau_{-}$corr. For this series of experiments, I used 1, 5, and $50 \mathrm{~ms}$ correlation time constants. Voltage output created as described above was limited to 0-5 V in order to protect the LED driver. By feeding the LED controller with the created fluctuating voltage, I obtained a fluctuating light with maximum light-intensity equals to $0.27 \mathrm{~mW} / \mathrm{mm}^{2}$.

Protocol (1) above was used to measure activation and deactivation time-constants of the various channelrhodopsins. Time-constants were measured here by fitting a single exponential to the activation/deactivation of currents elicited by light steps, from stimulus onset/offset to peak/steady-state current, respectively.

Protocol (2) was used to calculate the power-spectrum density (PSD) of photocurrents in response to light-chirps. PSD was calculated using Welch windows of $50 \%$ overlap with 16384 points (empirically determined, so that frequency resolution and curve smoothness was optimized). Power density was normalized by the maximum power. In order to estimate the cutoff frequencies of the different light responses, I compared the calculated PSD with the frequency-response of digitally synthetized single-pole RC filters having different cutoff frequencies.

Protocol (3) was used to test the current reproducibility across cells, and to estimate the impulse-response function (IRF) of the different channelrhodopsins in order to test the 
predictability of the induced currents. The first was calculated by finding the Pearson correlation coefficients of sample photocurrents across different cells. The latter was calculated by taking the inverse Fourier transform $F^{-1}()$ of the ratio of the Fourier transforms of the photocurrents $F(I(t))$ and the Fourier transform of the stimulus $F(S(t))$, i.e., $I R F=F^{-1}(F(I(t)) / F(S(t)))$. Predictability was then tested by comparing the measured currents with the predicted currents, calculated using linear-system theorem: convolving the fluctuating voltages with the impulse response function of a given channelrhodopsin. A quantification of the predictability was obtained by calculating the Pearson correlation coefficient between measured and predicted currents.

\subsection{Experiments in brain slices}

\subsubsection{Solutions used in brain slices experiments}

- aCSF (Artifical Cerebrospinal Fluid). Solution with which brain slices were maintained and recorded in. Composition (in mM): $\mathrm{NaCl}$ (125), $\mathrm{KCl}$ (4), $\mathrm{NaHCO}_{3}$ (26), Glucose (10), $\mathrm{MgCl}_{2}$ (1.3), $\mathrm{CaCl}_{2}$ (2). Osmolarity 295-305 m0sm. pH 7.4 after carbogen $\left(95 \% \mathrm{O}_{2}, 5 \% \mathrm{CO}_{2}\right)$ saturation. Filter-sterilized after prepared.

- Blocking solution. Used in staining protocols. Composition in PBS: 10 \% Normal horse serum (v/v), $0.1 \%$ Triton X-100 (v/v).

- Cutting aCSF. Solution in which brain slices were cut in. Composition (in mM): $\mathrm{NaCl}$ (125), $\mathrm{KCl}$ (2.5), $\mathrm{NaHCO}_{3}$ (26), $\mathrm{NaH}_{2} \mathrm{PO}_{4}$ (1.25), Glucose (25), Ascorbic Acid (0.4), Lactate (4), $\mathrm{MgCl}_{2}$ (1), $\mathrm{CaCl}_{2}$ (2). Osmolarity 315-325 m0sm. pH 7.4 after carbogen (95 $\% \mathrm{O}_{2}, 5 \% \mathrm{CO}_{2}$ ) saturation. Filter-sterilized after prepared.

- Lysis buffer. Used to digest animal biopsies for genotyping. Composition (in mM): $\mathrm{NaCl}$ (200), Tris pH 8.5 (100mM), EDTA (5mM), $10 \mathrm{ml}$ of $10 \%$ SDS.

- Mounting solution. Used when transferring brain slices to glass slides after staining. Composition in PBS: $0.2 \%$ Gelatin (w/v), $0.15 \%$ Triton X-100 (v/v). In order to prepare it, mix the gelatin with half the PBS and heat up for $1 \mathrm{~min}$ in the microwave. Add the other half of PBS and the Triton X-100.

- PBS (phosphate-buffered saline). Used in multiple protocols (e.g., perfusions, stainings, etc.). Composition (in mM): $\mathrm{NaCl}$ (140), $\mathrm{KCl}$ (2.7), $\mathrm{Na}_{2} \mathrm{HPO}_{4}$ (10), $\mathrm{KH}_{2} \mathrm{PO}_{4}$ (1.8). pH adjusted to 7.3. Filter-sterilized after prepared. 
- Potassium-gluconate internal solution. Pipette solution in patch-clamp recordings in brain slices. Composition (in mM): K-Gluconate (135), $\mathrm{KCl}(10), \mathrm{NaCl}$ (4), $\mathrm{Na}_{4} \mathrm{EGTA}$ (0.1), Mg-ATP (1), Na-GTP (0.3), HEPES (10), $\mathrm{Na}_{2}$-Phosphocreatine (0.5). Osmolarity 285-290 m0sm. pH adjusted to 7.3. Filter-sterilized after prepared.

\subsubsection{Mouse lines}

All experiments were performed in according with institutional and state regulations. Tissues from animals of two different mouse lines (NKTDTO and PVAI32; see below) were used for experiments in acute brain slices. All animals were kept in the animal facility of the Max Planck Institute of Experimental Medicine, in standard $12 \mathrm{~h}$ light regime with water and food ad libidum. All animals used in experiments were 3-7 weeks old and of either sex. In total, 16 animals were used in these experiments.

NKTDTO animals supposedly allow the targeting of a very restrict population of GABAergic interneurons called axo-axonic, or chandelier cells (Taniguchi et al., 2013). In this mouse line, the red fluorescent protein tdTomato is expressed in a Cre- and tamoxifendependent manner in cells expressing the transcription factor Nkx2.1. This transcription factor is involved in the differentiation of pallidal structures and in the development of GABAergic neurons that migrate from the pallidum into the cortex (Sussel et al., 1999). NKTDTO animals were obtained by crossing Nkx2-1tm1.1(cre/ERT2)Zjh/J animals (also known as Nkx2.1CreER ${ }^{\mathrm{T} 2}$, Taniguchi et al., 2013; The Jackson Laboratory stock \#014552) with B6;129S6-Gt(ROSA)26Sortm9(CAG-tdTomato)Hze/J (also known as Ai9, Madisen et al., 2010; The Jackson Laboratory stock \#007905). Nkx2.1CreERT2 males were obtained directly from the Jackson Laboratory and, after in-vitro fertilization performed by the transgenic core facility of the animal house of the Max Planck Institute of Experimental Medicine, a new colony was established. Ai9 animals used for breeding were kindly donated by Prof. Dr. Klaus Armin Nave (Max Planck Institute of Experimental Medicine).

Nkx2.1CreER ${ }^{\mathrm{T} 2}$ animals are transgenic animals in which the DNA sequence of the tamoxifen-dependent CRE-ER ${ }^{\mathrm{T} 2}$ recombinase was inserted downstream of the promoter/enhancer elements of the Nkx2.1 transcription factor (Taniguchi et al., 2011). In these animals, the CRE-ERT2 recombinase expression follows the pattern of the Nkx2.1 expression, i.e., it is expressed only in progenitor cells of the medial ganglionic eminence, from about embryonic day 10 (E10) to a few postnatal days (Taniguchi et al., 2011; Taniguchi et al., 2013). 
The CRE recombinase catalyzes recombination between two homologous 34-basepair DNA sequences called loxP (Feil et al., 1996; Metzger et al., 1995). The loxP sequence, by its turn, is present in Ai9 animals, in which a loxP-flanked stop codon is upstream of the DNA sequence of the red fluorescent protein tdTomato. In these animals, when the stop codon is excised by CRE, the expression of tdTomato is driven by the synthetic ubiquitous promoter CAG (Madisen et al., 2010). Therefore, by crossing Nkx2.1CreER mice with Ai9 mice and upon administration of tamoxifen during pregnancy, one can express tdTomato in medial ganglionic eminence-derived cells (which comprises a subpopulation of GABAergic cells in the cortex; Taniguchi et al., 2013).

PVAI32 animals were kindly donated by Dr. Sonja Wojcik, from the Max Planck Institute of Experimental Medicine. These animals express the light sensitive ion channel channelrhodopsin-2 in parvalbulmin expressing neurons (Fuchs et al., 2007; Madisen et al., 2012). Parvalbulmin is a $\mathrm{Ca}^{2+-b i n d i n g}$ protein exclusively expressed in fast spiking basket cells and, at least partially, chandelier cells (Kawaguchi and Kubota, 1997; Taniguchi et al., 2013). These animals were obtained by crossing PV-Cre animals (Fuchs et al., 2007) with B6;129S-Gt(ROSA)26Sortm32(CAG-COP4*H134R/EYFP)Hze/J mice (also known as Ai32, Madisen et al., 2012; The Jackson Laboratory stock \#012569). Ai32 animals express channelrhodopsin-2 fused with EYFP under the CAG promoter. A loxP-flanked stop codon upstream of the channelrhodopsin sequence assures that only Cre-expressing cells will express the channel. For the breedings, males heterozygous for the PV-Cre transgene and heterozygous or homozygous for Ai32 were bred with PV-Cre negative (wild-type), Ai32 heterozygous or homozygous females. Animals used in the experiments were positive for PVCre and either heterozygous or homozygous for Ai32.

\subsubsection{Tamoxifen induction and caesarian sections in NKTDTO animals}

NKTDTO breeding pairs were set up by crossing heterozygous Nkx2.1CreER ${ }^{\text {T2 }}$ male mice with either $\mathrm{Nkx2.1CreER^{ \textrm {T } 2 }}$ heterozygous or wildtype female mice. All mice were homozygous mutant (-/-) for the tdTomato gene. In order to control for the developmental stage of pregnant animals, breeding females were checked every morning for vaginal plugs after the breeding cage was set up. The day the plug was detected was considered 0.5-day in development. Expression of tdTomato was induced via tamoxifen gavage of the pregnant female $(0.3 \mathrm{mg}$ of tamoxifen in corn oil/30 g body weight) at embryonic day 17.5 (E17.5). Tamoxifen (Sigma-Aldrich) was prepared by overnight incubation under shaking at $37^{\circ} \mathrm{C}$ in corn oil. After incubation, if necessary, solution was sonicated at $37^{\circ} \mathrm{C}$ for 10 to $25 \mathrm{~min}$. 
A drawback of tamoxifen induction during pregnancy is the risk of the induction of pre-term delivery, and the cannibalism associated to it, which can severely reduce the number of animals available for experiments. While non-induced animals from this mouse line usually deliver at E20, induced females tended to delivery at E19. Nearly all the females that delivered by themselves at E19 or earlier displayed cannibalism and ate their offspring. In order to prevent this, I performed caesarian sections in the induced females at late E18 or early E19 and the offspring was put to a NMRI-line foster-mother. Caesarian sections were performed following standard procedures (Murphy, 1993). Briefly, timed-pregnant females were sacrificed by cervical dislocation followed by laparotomy on a pre-warmed pad after disinfection of the skin with povidone-iodine. The exposed uterus was then removed by cutting the oviducts and the cervix and cut into smaller pieces, each containing a single pup. Each pup was then gently squeezed out of the uterine tube onto a pre-warmed damped tissue and the membranes around it carefully removed. Gently, the chest of the pups was repeatedly squeezed and the nose and mouth were cleaned from any fluid with a slightly damped delicate paper tissue. This procedure was repeated until they were breathing consistently and exhibited the typical pinky color of newborns. Pups were then gently rubbed in bedding from the foster-mother's cage and mixed with the foster mother's litter. The NMRI foster mother must have had its offspring up to two days before the new pups are mixed.

\subsubsection{Genotyping}

All animals were genotyped before the experiments, and the same digestion and amplification protocols were used for both mouse lines.

\subsubsection{1 $\quad$ Sample digestion and DNA purification}

Samples used in digestion were either a piece of the tail or pieces of the ear obtained from ear punches. The digestion protocol consisted in the incubation of the samples in lysis buffer (see Section 2.2.1) with proteinase K $(100 \mu \mathrm{g} / \mathrm{ml}$ of buffer) for a minimum of $2 \mathrm{~h}$ (and maximum overnight) at $55{ }^{\circ} \mathrm{C}$ in a tabletop shaker (at $1000 \mathrm{rpm}$ ). Samples were then centrifuged at $13200 \mathrm{rpm}$ for $5 \mathrm{~min}$ in a standard tabletop centrifuge and the supernatant was transferred to a new tube. Working on ice, $300 \mu \mathrm{l}$ of isopropanol were added to each tube and another 15 min centrifugation was made in order to pellet the DNA. Supernatant was discarded and two successive washes (5 min each, and centrifugation at same speed as before) with $500 \mu \mathrm{l}$ of $80 \%$ ethanol were performed. Supernatant was discarded after each wash, and, at the end of the second wash, the DNA pellet was allowed to dry for about 5 min. 
DNA was dissolved in $100 \mu \mathrm{l}$ of endonuclease-free water and kept at $-20{ }^{\circ} \mathrm{C}$ until further processing.

\subsubsection{2 $\quad \underline{\text { Polymerase chain reaction and gel electrophoresis }}$}

Standard polymerase chain reaction (PCR) was performed under the same conditions for all mouse lines. Specific primers for each line (Table 1) and adjustments in the total volume of water required were necessary in each case. $20 \mu \mathrm{l}$ reactions were set up in $0.2-\mathrm{ml}$ PCR tubes, loaded with $2 \mu \mathrm{l}$ of purified DNA, $4 \mu \mathrm{l} 5 \mathrm{x}$ Taq Polymerase Buffer (OneTaq ${ }^{\circledR}$ Standard Reaction Buffer; New England Biolabs Inc.), $0.4 \mu \mathrm{l}$ dNTP mixture, $0.2 \mu \mathrm{l}$ of each primer (stock solution: $100 \mathrm{pmol} / \mu \mathrm{l}$; see primer sequences below), $0.4 \mu \mathrm{l}$ DNA Taq Polymerase (OneTaq® DNA Polymerase; New England Biolabs Inc.) and water to complete $20 \mu \mathrm{l} /$ tube. PCR was performed in standard thermocycler programmed with the following protocol:

i. $96^{\circ} \mathrm{C}, 30$ seconds

ii. $57-59^{\circ} \mathrm{C}, 45$ seconds

iii. $72^{\circ} \mathrm{C}, 1$ minute (repeat i-iii 29 times)

Table 1. Primers used for genotyping

Primer ID Primer sequence (5'> 3')

\begin{tabular}{cc}
\hline \multicolumn{2}{c}{ NKTDTO Animals } \\
\hline Nkx-Primer 1 & GCCTCCACTCAAGCCAATTA \\
Nkx-Primer 2 & CCTGGCCCTGTCTGTACG \\
Nkx-Primer 3 & ATGTTTAGCTGGCCCAAATG \\
TDTO-Primer 1 & AAGGGAGCTGCAGTGGAGTA \\
TDTO-Primer 2 & CCGAAAATCTGTGGGAAGTC \\
TDTO-Primer 3 & GGCATTAAAGCAGCGTATCC \\
TDTO-Primer 4 & CTGTTCCTGTACGGCATGG \\
& \\
& PVAl32 Animals \\
\hline PVCre-Primer 1 & CCAGGCTAAGTGCCTTCTCTACA \\
PVCre-Primer 2 & GACACTGCAGCGCTGGTCAT \\
Ai32-Primer 1 & AAGGGAGCTGCAGTGGAGTA \\
Ai32-Primer 2 & CCGAAAATCTGTGGGAAGTC \\
Ai32-Primer 3 & ACATGGTCCTGCTGGAGTTC \\
Ai32-Primer 4 & GGCATTAAAGCAGCGTATCC
\end{tabular}

$10 \mu \mathrm{l}$ of the PCR product were ran in $1.5 \%$ agarose at $100-120 \mathrm{~V}$ for approximately one hour. 


\subsubsection{Preparation of brain slices}

Acute brain slices were prepared similarly to da Silva et al (2015). Animals were anesthetized with a mixture of ketamine $(100 \mathrm{mg} / \mathrm{kg}$ of body weight) and xylazine $(20 \mathrm{mg} / \mathrm{g}$ body weight), injected intraperitoneally, and decapitated with a guillotine. The head was then submerged in ice-cold carbogen-saturated cutting aCSF (see section 2.2.1) and a craniotomy was performed. For this, the dorsal skin of the head was longitudinally cut from the neck until the snout and the skull covering the cerebellum was removed by means of a transversal cut at the junction of the parietal and interparietal bones. Using a delicate iris scissor, a medial longitudinal cut through the medial suture, from the most caudal part of the remaining skull until the mid of the parietal bones was made and each parietal bone was removed laterally with a forceps. With a bone cutter, the frontal bones were broken at the level of the orbits and were carefully removed, in order not to damage the olfactory bulb. The brain was finally extracted and chopped onto a smaller block by making a coronal cut immediately caudal to the diencephalon. This coronal cut served as the basis through which the brain block was glued with cyanoacrylate glue onto the vibratome cutting chamber, which was subsequently filled with cutting aCSF. Five to six 250-300 $\mu$ m-thick slices, starting from the frontal cortex, were cut in a VT1200S vibratome (Leica; speed $0.1 \mathrm{~mm} / \mathrm{s}$, amplitude $1.45-1.60 \mathrm{~mm}$ ) and immediately placed in carbogen-saturated recording aCSF (see section 2.2 .1 ) at $35^{\circ} \mathrm{C}$. Slices were kept at this temperature throughout the whole experiment. After a minimum of $1.5 \mathrm{~h}$ after cutting, one slice at a time was chosen for recording.

\subsubsection{Patch-clamp experiments in brain slices}

The chosen slice was put in a heated recording chamber (PH6; Warner Instruments) and was held in place with a slice hold-down (SHD-27LH/15; Warner Instruments). Carbogen-saturated aCSF was warmed by means of an in-line heater (HPT-2; Alasciences) and constantly perfused by gravitation through the recording chamber at a flow rate of 1-2 $\mathrm{ml} / \mathrm{min}$. Both, the recording chamber and the in-line heater were controller by a TC-20 dual channel temperature controller (NPI). Temperature in the recording chamber was kept at $36 \pm 1^{\circ} \mathrm{C}$ and was monitored via a thermistor placed $3-5 \mathrm{~mm}$ from the recording site. The recording chamber was positioned under an upright Axio Examiner.D1 microscope (Zeiss) equipped with a 10x W N-Achroplan and a W Plan-Apochromat 40x/1.0 DIC objective. The microscope was equipped with $900 \mathrm{~nm}$ infrared differential interference contrast optics (IRDIC) and with a multi-colored LED-controlled illumination system (pE-4000; CoolLed). Simultaneous infrared and fluorescent images could be obtained by means of the separation 
of light wavelengths with custom-designed dichroic mirror and filter sets and two cameras. Infrared images were obtained with a monochrome camera (vx44; PCO CCD Imaging) and displayed in a black and white screen. Epifluorescent images were obtained with a CCD camera (MD061RU-SY; Ximea) connected to a computer screen via an USB 3.0 cable. Epifluorescence camera was controlled via the open-source software $\mu$-Manager (Edelstein et al., 2010). Healthy neurons were selected based on their appearance in the infrared channel, following standard techniques (Moyer and Brown, 1998). All recordings were made under synaptic blockade with the following blockers: (1) picrotoxin (30 $\mu$ M; Sigma-Aldrich), (2) NBQX (10 $\mu \mathrm{M}$; Sigma-Aldrich), and (3) DL-2-Amino-5-phosphonopentanoic acid (30 $\mu \mathrm{M}$; Sigma-Aldrich). Patch-clamp recordings were made using standard techniques. Briefly, patchclamp pipettes made from borosilicate glass capillaries (PG10165-4, World Precision Instruments) were pulled in a vertical pipette puller (PIP 6 Micropipette Puller, HEKA), so that their resistances were between 3 and $6 \mathrm{MOhm}$, as measured with a potassium gluconate internal solution (see section 2.2.1). Whole-cell patch-clamp recordings in current clamp mode targeting the soma were performed using an EPC-10 Double USB amplifier, controlled by Patchmaster software (both from Heka). Capacitance and series resistance were adjusted after entering whole-cell mode, in voltage-clamp. After changing to current-clamp mode, bridge balance was set to $100 \%$ and $10 \mu$ s. Signals were low-pass filtered at $8.8 \mathrm{kHz}$ and digitized at 20 (current-steps protocol, see below) or $100 \mathrm{kHz}$ (fluctuating current protocols). Data analysis was performed offline in custom-written programs in Matlab (Matlab 2014b, Mathworks) and Igor (Igor Pro 6, Wavemetrics). Voltages here reported have not had the liquid junction potential corrected. For the combination of solutions used in brain slices patch-clamp recordings, the calculated liquid junction potential is approximately $-14 \mathrm{mV}$.

\subsubsection{Current injection protocols}

\section{$\underline{\text { Electrical type classification }}$}

In order to obtain information regarding the specific cell type of the recorded cells, a protocol consisting of successive $500 \mathrm{~ms}$ depolarizing $15 \mathrm{pA}$ current steps from resting potential was applied. The number of sweeps varied from cell to cell, but current was increased until at least a value $50 \%$ greater than rheobase value. For analysis, interneurons were classified based on their electrical types, or e-types, as suggested in the literature (Ascoli et al., 2008; Druckmann et al., 2013). Briefly, two aspects of the response to depolarizing suprathreshold currents were taken into account: the response at the onset of the stimulus and the "steady-state" response, i.e., the firing pattern after stimulus onset. Onset response can be classified as "burst", "delayed" and "continuous", while steady-state 
response can be "fast spiking", "non-adapting non-fast spiking", "adapting", "irregular spiking", "intrinsic burst firing", and "accelerating" (Ascoli et al., 2008; Druckmann et al., 2013). Within the fast spiking subset, interneurons often exhibit a "stuttering" pattern, which is distinct from the burst, continuous, and delayed patterns (Ascoli et al., 2008; Druckmann et al., 2013). The classification was performed using either the rheobase or the 1.5 times rheobase responses. Following the classification, a few parameters of the spikes or spike trains were extracted and compared between the identified e-types. These were the following:

- Spike threshold: defined as the voltage value at which the slope of the phase plot $(\mathrm{dV} / \mathrm{dt}$ vs $\mathrm{V}$ plot, where $\mathrm{V}$ is the voltage during an action potential) crosses 30 $\mathrm{V} / \mathrm{s}$.

- Afterhyperpolarization magnitude: the magnitude of the voltage difference between spike threshold and the negative peak of the hyperpolarizing spike phase.

- Spike onset rapidness: the slope at the spike threshold in the phase plot

- Spike half-width: width (in ms) at the half-maximal spike amplitude, where maximal spike amplitude is the amplitude between spike threshold and peak.

- Frequency: defined as the inverse of the average interspike interval in a spike train

- Interspike interval ratio: defined as the ratio of the last and first interspike intervals

\section{Gain function calculation}

In order to access the frequency response function of the recorded neurons, DC current was injected into the neurons, so that their resting potential was kept around -59 to $64 \mathrm{mV}$. A 30-second-long fluctuating current with 0 mean and a defined standard deviation, mimicking in vivo activity and consisting of a stochastic process similar to an OrnsteinUhlenbeck process (Destexhe et al., 2001), was synthetized in Igor as described in section 2.1.3.1, but now passing the signals through filters with time constant $\tau_{-}$corr equals to either 5 or $25 \mathrm{~ms}$. The standard deviation of the constructed noise stimuli was adjusted for each cell, so that its average firing rate was typically between 2 and $7 \mathrm{~Hz}$. 
For each repetition of this protocol, a noise created with a different random seed was used. Current injection episodes consisted of 30s of injection intercalated by $15 \mathrm{~s}$ without injection, and a variable number of repetitions were used in each neuron. Shifts of the membrane potential to values greater than $-60 \mathrm{mV}$, spikes overshooting less than $20 \mathrm{mV}$ and series resistance greater than $30 \mathrm{MOhm}$ were used as indicatives of deteriorated recordings.

\subsubsection{Calculating the frequency-response function}

In order to assess the frequency-response function of the neurons, I used a similar approach to Higgs and Spain (2009). Essentially the method consists in taking the ratio of the Fourier transform of the spike-triggered average (STA) of each cell and the Fourier transform of the autocorrelation of the injected noise (Figure 1).

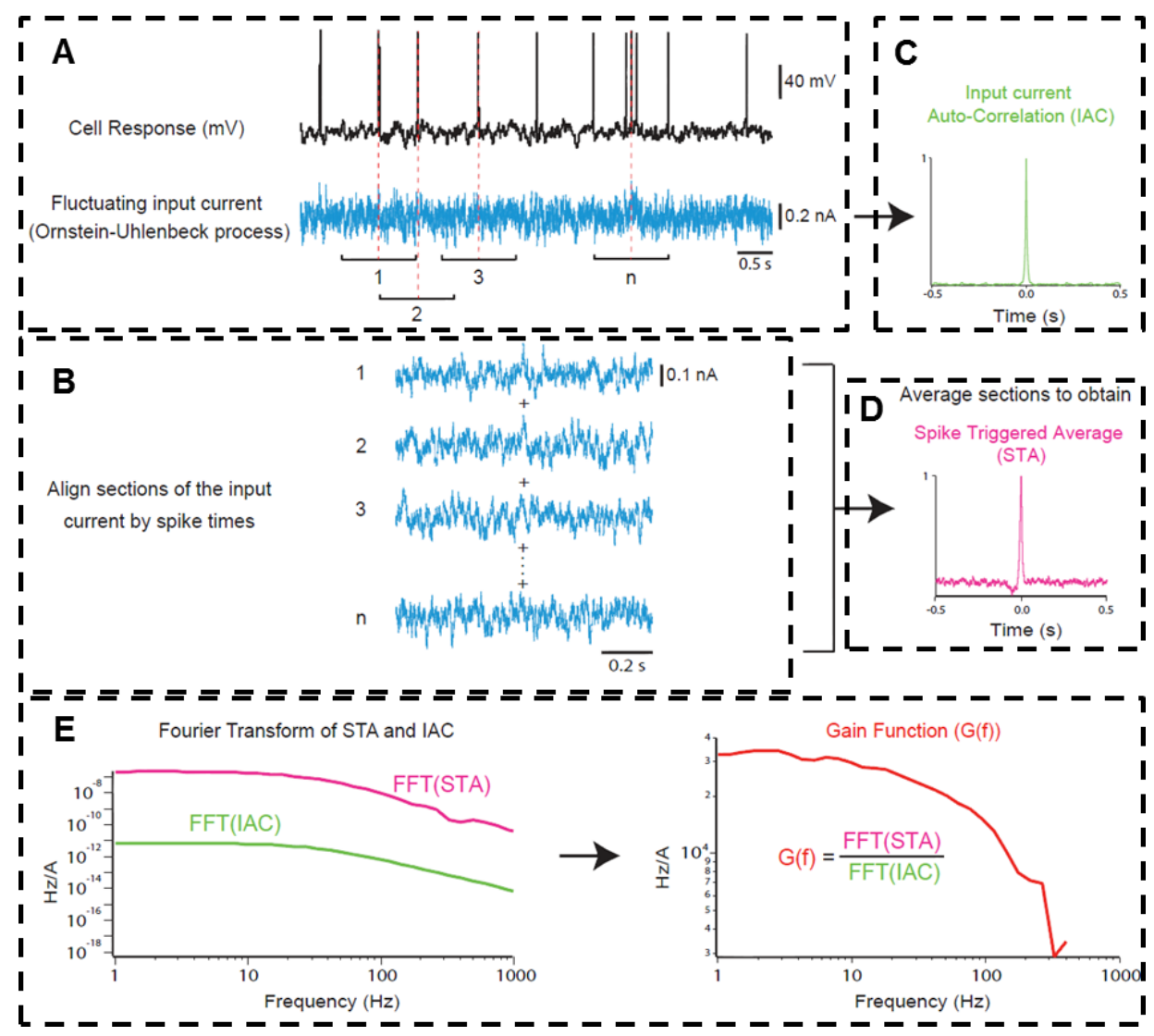

Figure 1. Calculation of the dynamic gain function. (A) A fluctuating current is somatically injected in neurons, and their voltage responses recorded. (B) For each recorded spike, a 500ms long segment of the injected current, centralized at the spike time, is obtained. Here, only 4 are indicated for readability reasons. (C) The input auto-correlation is calculated, and (D) all the input segments in B averaged, resulting in a spike-triggered average. (E) The Fourier transform of both, the input auto-correlation and STA, are calculated, and the gain is obtained from the ratio of these Fourier transforms. Data in the figure was kindly given by Dr. Elinor Lazarov. Figure composition based in Ilin et al. (2013), and made with contribution from Dr. Carolina León-Pinzón. 
Spike-triggered averages were calculated first by mapping spike-times (defined as the time in which the voltage during an action potential crosses upwardly $0 \mathrm{mV}$ ) onto the respective stimulus current trace. For each spike-time mapped onto the current, a current window of $1 \mathrm{~s}$ centered on the spike-time was detected and averaged across all spikes in a given trial. STAs among different trials for the same cell were normalized by the trial's average firing rate and trial input current's standard deviation. STAs from all cells in the same condition were pooled together, averaged and multiplied by the global average standard deviation (i.e., the average standard deviation among all trials and cells). The input autocorrelation function for each trial was calculated, averaged, and normalized by the average input variance across cells.

Both, STA and autocorrelation were then split at time 0 (corresponding to the peakvalue of both), and the last half of each shifted to the beginning of the trace. In order to improve signal-to-noise ratio, the STA was filtered in the complex domain by using a Gaussian-window $w\left(f^{\prime}\right)$, centered at frequency $f^{\prime}=f$, with a standard deviation of $f / 2 \pi$,

$$
w\left(f^{\prime}\right)=\frac{1}{\sqrt{2 \pi} \cdot \frac{f}{2 \pi}} \cdot \exp \left[-\frac{1}{2}\left(\frac{f^{\prime}-f}{f / 2 \pi}\right)^{2}\right]
$$

Hence, the Gaussian-window filtered STA $\left(\mathrm{STA}_{w}\right)$ becomes

$$
\operatorname{STA}_{\mathrm{w}}(f)=\frac{\int \operatorname{STA}\left(f^{\prime}\right) \cdot w\left(f^{\prime}\right) \cdot d f^{\prime}}{\int w\left(f^{\prime}\right) \cdot d f^{\prime}} .
$$

The dynamic gain function $G(f)$ is calculated by taking the ratio of the Fourier transform of the Gaussian-window filtered STA $\left(F\left(S T A_{\mathrm{w}}(f)\right)\right.$ and the Fourier transform of the autocorrelation of the input current $\left(F\left(c_{\mathrm{ss}}(\tau)\right)\right.$,

$$
c_{\mathrm{ss}}(\tau)=\langle s(t) s(t+\tau)\rangle
$$

where $c_{\mathrm{ss}}(\tau)$ is the autocorrelation of the input current, $\mathrm{s}(\mathrm{t})$ is the input current and $\tau$ the time lag.

The gain function G(f) thus becomes

$$
G(f)=\frac{\left|F\left(S T A_{w}(f)\right)\right|}{\mid\left(F\left(c_{\mathrm{ss}}(\tau)\right) \mid\right.} .
$$





\section{Chapter 1: Optogenetic Tools for Characterizing Neuronal Transfer Functions}

\subsection{Chapter 1: Introduction}

The notion that the brain is formed by an immense number of different cell types dates back to the works of Santiago Ramon y Cajal, and suggests that specific cell types are involved in specific tasks within their circuits. For decades, such experiments aiming at uncovering the role of defined neuronal subtypes or populations on behavior remained largely illusory. The discovery of channelrhodopsins immediately aroused the awareness of the neuroscience community, exactly for making such long-sought experiments a reality.

\subsubsection{Optogenetic revolution}

Optogenetics can be broadly defined as the use of hybrid methods based on optics and genetics to manipulate well-defined biological events in biological systems from cells to behaving animals (Deisseroth, 2011, 2015). Even though all components required for such manipulation were known since decades, only about 10 years ago, when Boyden et al. (2005) were able to remotely control neuronal activity with light, that the scientific community started to realize its revolutionary power. By surpassing many of the limitations of other techniques, optogenetics have allowed scientists to causally investigate a broad range of problems never addressed before.

Even though the paper by Boyden et al. (2005) is considered by many the starting point of optogenetics, after which the field flourished, another group 3 years before them had already used a combination of genetically encoded and light-sensitive actuators to control biological functions, in this case to sensitize neurons to light (Zemelman et al., 2002). A possible reason for this three-year hiatus is probably the fact that the method employed in Zemelman et al. (2002) relied on a complex, three-component system to achieve control over 
neurons. The Boyden et al. method, however, relied in a single light-sensitive protein: the channelrhodopsin (ChR).

Channelrhodopsins are members of the family of microbial rhodopsins, proteins found in algae and archaebacterial which, in response to light, allow the movement of charges across the membrane. Other members of this family are the bacteriorhodopsins, which are light-activated proton pumps, and halorhodopsins, which pump chloride into cells upon light stimulation. The first report on the actual identity of these molecules came about in 1971, with the discovery of the bacteriorhodopsin (Oesterhelt and Stoeckenius, 1971). For about 30 years the neuroscience community was oblivious to the enormous potential of such proteins, until the discovery of channelrhodopsins, which became a complete game-changer in neuroscience (Nagel et al., 2002; Nagel et al., 2003).

\subsubsection{Structure of Channelrhodopsins}

Channelrhodopsins are ion channels first found in the green algae Chlamydomonas reinhardtii (Nagel et al., 2002). Located in the eyespot of algae, their natural function in these organisms is to trigger phototactic or photophobic responses in the cell, by direct or indirect activation of flagella (Sineshchekov et al., 2002). They consist of 7 -transmembrane domain (H1 to H7) proteins that covalently bind the light-isomerizable chromophore retinal through a protonated Schiff base (Nagel et al., 2002; Nagel et al., 2003). Most of what is known about the detailed structure of channelrhodopsins comes from high-resolution X-ray crystallography on a fully dark-adapted chimera of the channelrhodopsin 1 and channelrhodopsin 2 (ChR1 and ChR2; C1C2 chimera) isoforms, consisting of the first 5 transmembrane domains of ChR1 and the last 2 of ChR2 (Kato et al., 2012). In the C1C2 chimera, the pore of the channel is formed between $\mathrm{H} 1, \mathrm{H} 2, \mathrm{H} 3$, and $\mathrm{H} 7$, and serves as a cation-selective permeation pathway (Kato et al., 2012). Primarily, ChRs are optimized for proton transport across the membrane, but other monovalent and divalent ions are also transported (Nagel et al., 2002; Nagel et al., 2003), being ion selectivity pH- and voltagedependent (Schneider et al., 2013).

In the channelrhodopsin 2, the retinal is bound to a conserved lysine (K257) in H7 via a protonated Schiff base. Two glutamate residues, E123 and E253, counterbalance the charge of the Schiff base (Schneider et al., 2015). By structural analysis of C1C2 and $\mathrm{pK}_{\mathrm{a}}$ calculations, it has been suggested that, in the dark-adapted state, E123 is protonated and E253 is deprotonated, indicating that the latter is a proton acceptor (Kato et al., 2012). As these residues are critical for the photocycle of the channel, targeted mutations on them usually 
have strong effects on the channel photocurrents (Berndt et al., 2011; Gunaydin et al., 2010). Other crucial residues in coupling the isomeric changes of the retinal to channel gating are the C128 and D156. Single mutations in either of these residues result in up to ten-fold deceleration of the channel opening and closing, bringing about the so-called step-function rhodopsins, channels that open upon blue-light stimulation and stay open until stimulated with green/yellow light (Bamann et al., 2010; Berndt et al., 2009). The T159 residue of the retinal binding pocket is another important amino acid determining the retinal binding. A replacement of T159 to cysteine results in a three- to ten-fold increase in the stationary component of the photocurrent, most likely due to an improvement of the retinal binding to the channel (Berndt et al., 2011; Prigge et al., 2012; Ullrich et al., 2013).

\subsubsection{Channelrhodopsin photocycle}

A full channelrhodopsin photocycle involves the absorption of a photon by the retinal chromophore, which causes its isomerization and triggers conformation rearrangements of the protein until its return to the original dark state (Figure 2).

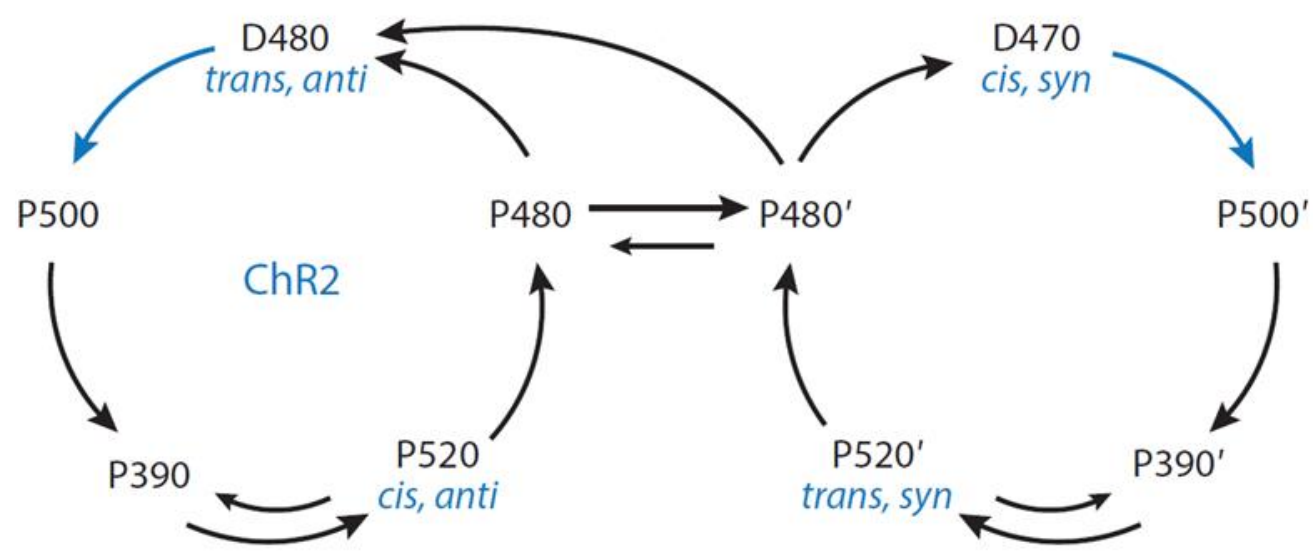

Figure 2. Photocycle model of channelrhodopsin-2. Two model photocycles for the two retinal isomerizations found in ChR2 (Schneider et al., 2015), as determined by spectroscopy. P520 intermediates are the conducting states. Blue arrow represents photon absorption.

Spectroscopy analysis of the photocycle identified multiple intermediate states. Upon light stimulation, retinal restructuration and initial changes in the protein structure form the nonconducting P500 intermediate (Nagel et al., 2003; Verhoefen et al., 2010). The Schiff base is then deprotonated and the P390 intermediate is formed, in which changes in the gates of the channel can already be detected (Schneider et al., 2015). However, the involvement of P390 in ion conduction is still under discussion (Bamann et al., 2008; Lorenz-Fonfria et al., 
2013). P390 is equilibrated with P520, the consensual conducting state exhibiting a reprotonated Schiff base (Ritter et al., 2008). When the channel closes, a change from P520 to P480 or P480' occurs and, finally, the channel relaxes to the dark state (Bamann et al., 2008). Other major conformational changes happen during dark state recovery in a timescale of seconds (Ritter et al., 2008). It has been shown that multiple retinal isomerizations occur in parallel in channelrhodopsins, with two independent photocycles involving the photoconversion of all-trans,15-anti retinal to 13-cis,15-anti retinal and 13-cis,15-syn retinal to all-trans,15-syn retinal, with the transition between the two cycles happening at the late stage of P480 intermediates (Schneider et al., 2015).

\subsubsection{Photocurrent properties}

Most studies of channelrhodopsins photocurrents have been carried out in Xenopus oocytes and human embryonic kidney (HEK) cells under voltage-clamp conditions. From such studies it has been shown that, typically, upon light stimulation, ChRs exhibit a fast peak current $\left(\mathrm{I}_{\mathrm{p}}\right)$ which decays to a stationary current $\left(\mathrm{I}_{\mathrm{s}}\right)$ in a process referred to as inactivation. This decay is thought to represent the desensitization of the channel through equilibration of two different conducting states and by accumulation of the late P480 nonconducting intermediates (Berndt et al., 2010; Nagel et al., 2003). When light-stimulation is over, currents decay biexponentially to baseline, which suggest the presence of at least two conducting states contributing to $I_{s}$ (Hegemann et al., 2005). In ChR2, total recovery to fully dark-adapted states requires several seconds, with stimulation in partly-adapted states resulting in smaller peak currents (Hegemann et al., 2005; Nagel et al., 2003; Schneider et al., 2015).

Most ChRs are primarily $\mathrm{H}^{+}$conductors (but see Govorunova et al., 2015), conducting also other monovalent and divalent cations, particularly at physiological conditions of low $\mathrm{H}^{+}$ concentrations. It has been shown that the relative conductance for various ChRs is as follows: $\mathrm{p}\left(\mathrm{H}^{+}\right)>>\mathrm{p}\left(\mathrm{Li}^{+}\right)>\mathrm{p}\left(\mathrm{Na}^{+}\right)>\mathrm{p}\left(\mathrm{K}^{+}\right)>\mathrm{p}\left(\mathrm{Rb}^{+}\right)>\mathrm{p}\left(\mathrm{Cs}^{+}\right) \approx \mathrm{p}\left(\mathrm{Ca}^{2+}\right)>\mathrm{p}\left(\mathrm{Mg}^{2+}\right)$ (Berndt et al., 2010; Lin et al., 2009; Nagel et al., 2003; Schneider et al., 2013; Schneider et al., 2015). Cations in a solution compete for binding and transport in a voltage- and $\mathrm{pH}$-dependent manner. Therefore, under conditions in which $\mathrm{H}^{+}$concentration is low, $\mathrm{Na}^{+}$is the main conducted (physiological) ion (Schneider et al., 2013). It is worth noting that $I_{p}$ and $I_{s}$ exhibit different cation selectivity, as observed by their distinct reversal potentials (Schneider et al., 2013). As the channel's conductance relies heavily on conditions such as $\mathrm{pH}$ and voltage, a universal description of this parameter is not possible for ChRs, and the experimental 
conditions must be clearly specified. It has been shown that, for ChR2 at an external pH 7.2 and membrane potential of $-60 \mathrm{mV}, \mathrm{I}_{\mathrm{p}}$ is mainly driven by protons, with $\mathrm{Na}^{+}$contributing to approximately one third of the current. In $\mathrm{I}_{\mathrm{s}}$, however, $\mathrm{Na}^{+}$is responsible for approximately $50 \%$ of the current (Schneider et al., 2013).

\subsubsection{Channelrhodopsin variants}

As explained above, certain amino acids in the retinal binding pocket or related to the isomerization process of retinal are determinant to the channel's function. Thus, mutations targeting these crucial amino acids have been shown to have a strong effect in the photocycle and, consequently, in photocurrents. Therefore, since 2005, a number of groups have been trying to modify or improve ChRs characteristics such as maximal conductance, ion selectivity, wavelength sensitivity and kinetics.

Estimated single-channel ChR2 conductance is on the order of tens to hundreds fS, which is considerably less than the common membrane channels in neurons (Bamann et al., 2008; Feldbauer et al., 2009; Lin et al., 2009; Nagel et al., 2003). Therefore, in order to control neuronal activity with ChRs, one must rely on high expression levels. Increases in singlechannel conductance are thus highly desirable. The mutation H134R in the wildtype ChR2 has been shown to exhibit a reduction in the inactivation levels, resulting in greater $\mathrm{I}_{\mathrm{s}}$ (Gradinaru et al., 2007; Nagel et al., 2005), which is important for the sustained activity of the channel. Mutations of E123 to alanine, glutamine or threonine speed up the photocycle of the channel, resulting in faster channels that became known as ChETAs (Berndt et al., 2011; Gunaydin et al., 2010), while a T159C mutation dramatically increases the photocurrent amplitudes (Berndt et al., 2011). The double mutant E123T/T159C is said to combine the high speed and sensitivity of E123T to the large photocurrents of T159C (Berndt et al., 2011). In order to compensate for the significant inactivation of ChR2, a chimera between ChR1 and ChR2, called ChEF was designed (Lin et al., 2009). With a further I170V mutation, ChIEF exhibits faster kinetics while retaining small inactivation (Lin et al., 2009). Other mutations aimed at shifting the ion selectivity of the ChRs. The mutation $\mathrm{L} 132 \mathrm{C}$ resulted in $\mathrm{CatCh}$, or $\mathrm{Ca}^{2+-}$ translocating channelrhodopsin, presenting a 6-fold higher $\mathrm{Ca}^{2+}$ permeability, faster kinetics and up to a 70-fold increase in light-sensitivity in comparison to ChR2 (Kleinlogel et al., 2011).

In addition to the screening of mutants, other labs opted for screenings on the transcriptome level of various species of algae (Klapoetke et al., 2014). With this approach, Klapoeteke et al (2014) isolated from the alga Stigeoclonium helveticum an ultra-fast, ultra- 
light-sensitive channelrhodopsin called chronos, reported to be fastest channelrhodopsin available. The same strategy also resulted in the discovery of GtARC2, the first naturally anion-conducting channelrhodopsin (Govorunova et al., 2015).

\subsubsection{Channelrhodopsin applications}

The main driving force pushing the development of optogenetic is neuroscience. Until the discovery of channelrhodopsins and the recognition of its power as a tool for controlling neurons, neuroscientists relied almost exclusively on electrical and chemical methods to artificially modulate neuronal activity, both having as a major downside their unspecificity with respect to targets. Optogenetics offered a way to circumvent such weakness, and therefore allowed neuroscientists to explore questions never tested before. The proof-ofconcept paper describing remote optogenetic stimulation of neurons was done in vitro, in cultured hippocampal neurons (Boyden et al., 2005). As early as 2007, however, the first reports on the direct effects of specific neuronal population on behavior came about (Adamantidis et al., 2007; Aravanis et al., 2007). Ever since, with the expansion of the optogenetic toolbox and discovery of other potential applications to this method, its scope has been expanded beyond uniquely that of controlling excitable cells.

While the main animal models for optogenetic interrogation of neural circuits have been rats and mice, optogenetic tools have been used in an increasingly wide variety of vertebrate and invertebrate species, such as the nematode Caenorhabditis elegans (Husson et al., 2013), the fruit fly (Ramdya et al., 2015), fishes (Thiele et al., 2014), songbirds (Roberts et al., 2012), nonhuman primates (Namboodiri and Stuber, 2016) and even human ex vivo retina (Busskamp et al., 2010). Optogenetics associated with the discovery of molecular markers for neuronal subtypes, such as parvalbulmin (PV)-, vasoactive intestinal peptide (VIP)-, or somatostatin (SOM)-positive interneurons have shed light on the specific functions of these cells on behavior (Pfeffer et al., 2013). PV+-interneurons have been shown to modulate the gain of layer2/3 pyramidal neurons in the visual cortex (Atallah et al., 2012), and to causally induce onset of gamma rhythm in the barrel cortex (Cardin et al., 2009). Also by means of optogenetic circuit interrogation, it has been shown that VIP+ interneurons are involved in a disinhibitory subcircuit in the medial prefrontal and auditory cortices, which also contribute to a gain modulation in pyramidal neurons (Pi et al., 2013), while SOM+ interneurons contribute to orientation selectivity in the visual cortex (Wilson et al., 2012). Besides in vivo experiments, channelrhodopsin stimulation has also been used as a model for studies about plasticity in vitro (El Hady et al., 2013; Wefelmeyer et al., 2015). 
In addition to experiments aiming at interrogating the function of certain cell types in brain computations or behavior, other researchers have used optogenetics to examine the function of single proteins in biochemical cascades, such as that of phosphoinositide 3-kinase (Toettcher et al., 2011) or G-protein coupled receptors (Airan et al., 2009).

Recently, our group developed an optogenetic-based method to investigate encoding properties of neurons in in vitro preparations: Continuous Dynamics Photostimulation (Neef et al., 2013), or CoDyPS, which promises to significantly facilitate the computation of neuronal gain functions.

\subsubsection{Continuous Dynamic Photostimulation - CoDyPS}

In general, excitatory optogenetic tools are used in neuroscience to precisely induce firing activity through suprathreshold optical stimulation, either with fast pulsed or step-like illumination. As most neuronal computations happen in the subthreshold regime, it is also desirable to gain precise control of subthreshold conductances, while giving the neuron autonomy to decide whether or not to fire action potentials. This paradigm has been shown to be fundamental for the characterization of neuronal frequency-response function (Higgs and Spain, 2009; Kondgen et al., 2008; Tchumatchenko et al., 2011). In order to facilitate the calculation of such neuronal property, our group has devised a noninvasive, optogeneticbased method, continuous dynamic photostimulation (CoDyPS), to precisely control neuronal subthreshold membrane potential in vitro (Neef et al., 2013). When associated to noninvasive extracellular recording methods, CoDyPS offers great advantage over standard, electrodebased methods used for gain calculation (Figure 3).

Essentially, CoDyPS relies on the linearity of ChRs light-response. By calculating the transfer function of the ChR used and using a linear system approach, one can predict with near $100 \%$ certainty what the ChR current will be given a certain light waveform (Figure 4; Neef et al., 2013). This way, under synaptic blockade (i.e., with glutamate and GABA blockers), one can assure that essentially the only subthreshold membrane fluctuations are the ones induced by ChR. Since the precise knowledge of the subthreshold potential is a required condition for the gain function estimation (see for example, Higgs and Spain, 2009; Tchumatchenko et al., 2011), CoDyPS can, thus, be used for such experiments. 


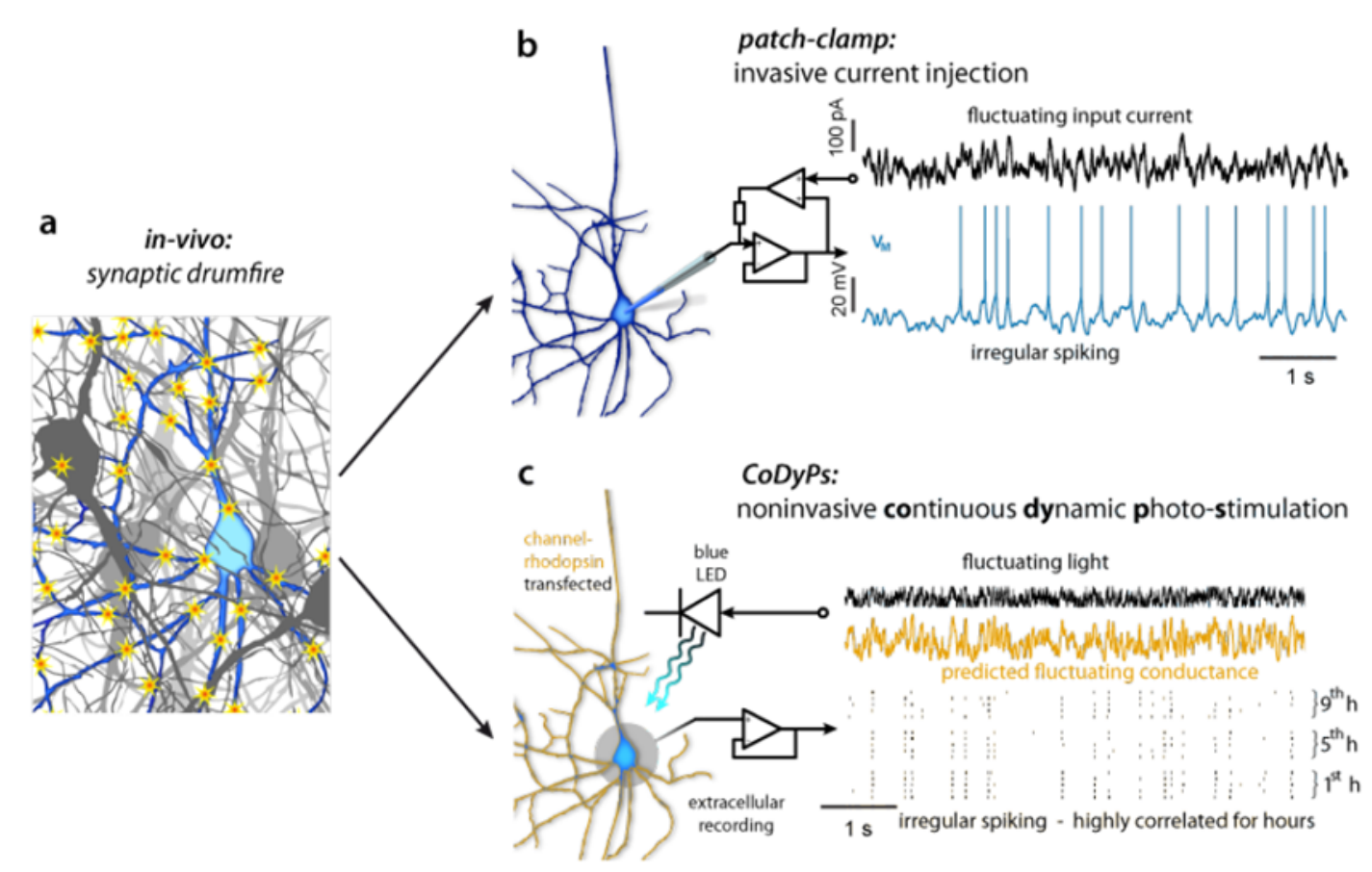

Figure 3. Different methods used to calculate neuronal gain function. (A) Neurons convert synaptic inputs in the order of thousands per second to spikes in the few hertz range. Neuronal gain function calculation sheds light on how neurons execute this transformation. (B) Traditionally, gain function is calculated based on intracellular recording methods, such as patch-clamp. Due to their invasiveness and the great number of spikes required, standard intracellular-based methods for calculating neuronal gain function are very laborious. (C) When associated to extracellular recordings, CoDyPS offers a totally noninvasive method, which speeds up gain function calculation by means of longer and simultaneous multi-cell recordings (Figure from Neef et al., 2013).

In the characterization of CoDyPS, two different channelrhodopsins, channelrhodopsin-2 and ChIEF, were used (Figure 5; Neef et al., 2013). ChIEF's main advantage over ChR2 is the larger $I_{s}$, due to the small inactivation caused by the I170V mutation (Lin et al., 2009). However, in terms of kinetics, both are equally fast (Figure 5C-E). In response to chirp stimulation, both channels behave as low-pass filters with $20 \mathrm{~Hz}$ cutoff frequency. By using faster channels, one can further optimize CoDyPS, allowing faster fluctuations and better control of the light stimulation. 
a

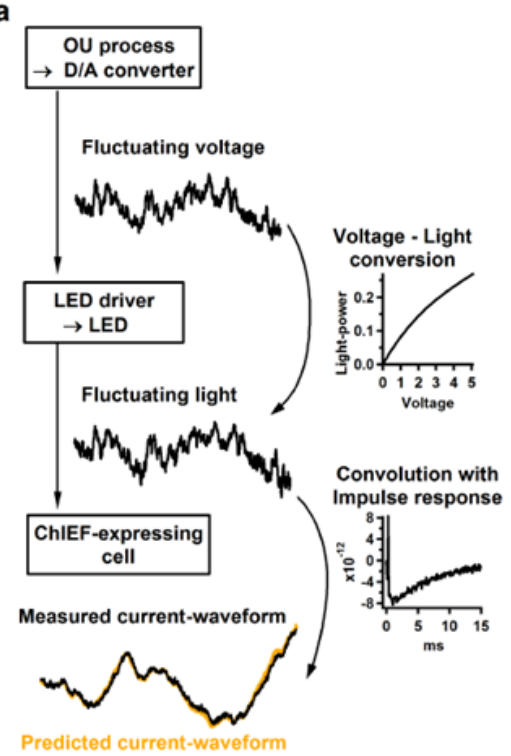

b

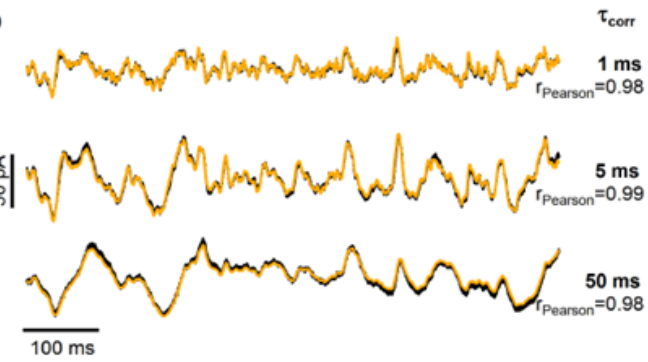

c

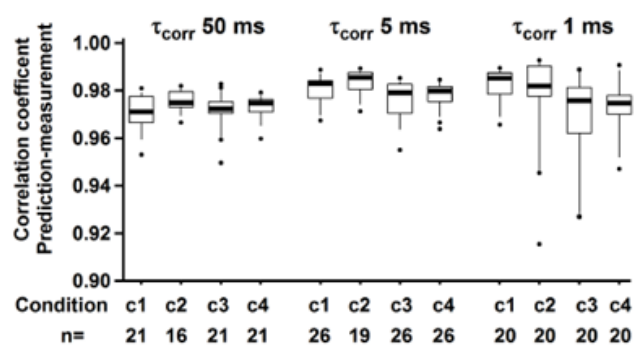

Figure 4. Prediction of photocurrents in CoDyPS. (A) Flow chart depicting the process for predicting photocurrents. Predicted current is calculated by convolving the fluctuating lightstimulus with the channelrhodopsin's impulse response function. (B) Samples of measured (black) and predicted (orange) currents for stimulus of different correlation times. (C) Correlation coefficient of predicted currents, for various stimulus conditions, indicating that ChRs' currents are highly predictable. (Figure from Neef et al., 2013).
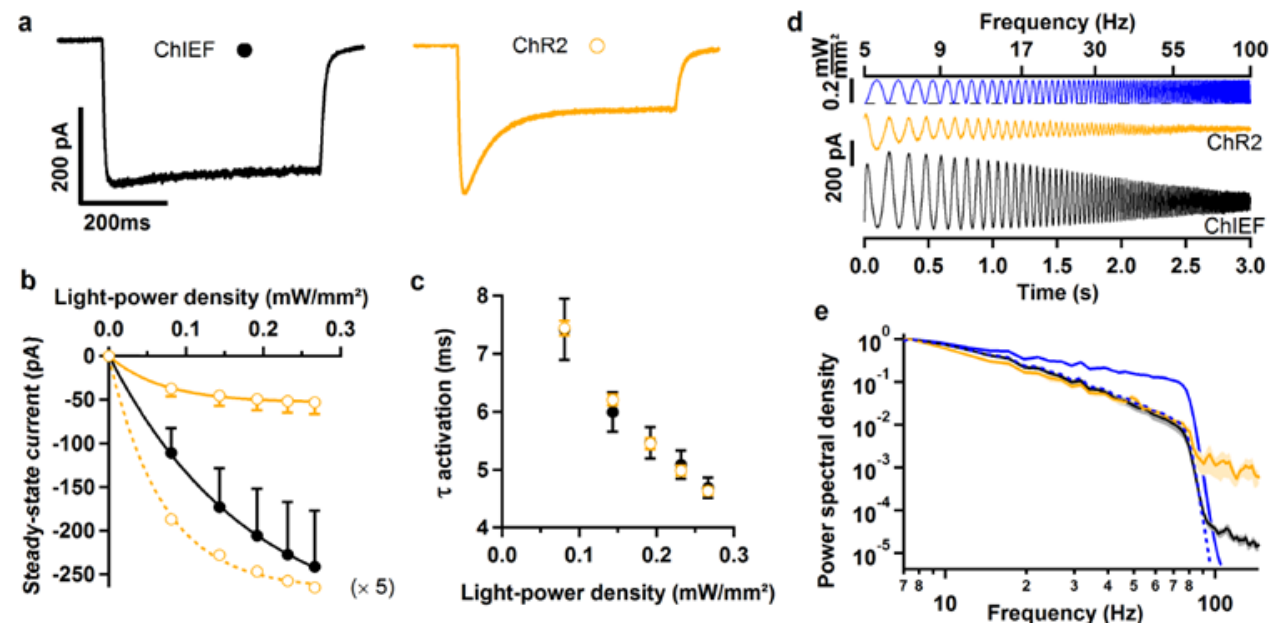

Figure 5. Characteristics of ChIEF and ChR2. (A) Sample traces of ChIEF (black) and ChR2 (orange) photocurrents. ChIEF exhibits much less inactivation, resulting in larger steadystate currents. (B) Relationship between steady-state current and light-power density. (C) Activation time-constant, highlighting the fact that both channels are equally fast and equally dependent on light intensity. (D). Chirp stimulus (blue) and ChR2 and ChIEF responses, indicating high attenuation of the photocurrents as stimulus frequency increases. (E) Power spectral density of chirp responses. Both channels exhibit a $20 \mathrm{~Hz}$ cutoff frequency. (Figure from Neef et al., 2013). 


\subsubsection{Goals in this project}

Given what I have expounded above, I sought to optimize CoDyPS by screening among available fast channelrhodopsins, namely chronos and ChETA E123T/T159C and the $\mathrm{Ca}^{2+-}$ permeable variant CatCh, in order to determine which one exhibits the best features for usage in dynamic stimulation paradigms. 


\subsection{Chapter 1: Results}

The discovery of channelrhodopsins and their application as tools to control neuronal activity opened up a vast range of long-sought experiments to interrogate genetically defined neuronal subpopulations and circuits (examples are Atallah et al., 2012; Cardin et al., 2009; Olsen et al., 2012; Pfeffer et al., 2013; Sohal et al., 2009). As its value as a revolutionary tool was promptly observed, in a few years interval researchers had at hand a number of lightsensitive protein variants, each one with its own strength and weakness (Mattis et al., 2012). It became paramount to understand each of these variants, so one could optimally select the one which would fit best for a particular set of experiments. Quickly it became obvious that fast channelrhodopsins would clearly confer advantages for many experiments in which stimulus rapidness and precision were required (Berndt et al., 2011; Gunaydin et al., 2010), and for this reason, a number of groups dedicated their research agenda to develop or discover variants exhibiting faster kinetics than the standard channelrhodopsin-2 (ChR2). Since the dynamic photostimulation our group developed, CoDyPS, can be limited by the performance of the channelrhodopsin in use, I started out testing some of the fastest channelrhodopsins available, searching the best fitted to our application. While fast kinetics is crucial, it is not the only property a channelrhodopsin must have in order to be used in experiments in which the light-induced conductance must be precisely known, such as for determining the frequency-response function of neuronal populations. High reproducibility and predictability are also of paramount importance in such cases. Here I evaluated how three fast variants of light-sensitive ion-channels, CatCh (Kleinlogel et al., 2011), ChETA variant E123T/T156C (ETTC; Berndt et al., 2011), and chronos (Klapoetke et al., 2014) compared to the widely used channelrhodopsin-2 (ChR2; Nagel et al., 2003) in terms of kinetics, reproducibility, and predictability.

\subsubsection{Current kinetics}

In order to evaluate the channels with respect to their rapidness, I first used a lightstep protocol consisting of 1 -s-long light stimulation with different intensities $(0.08,0.14$, $0.19,0.23$, and $0.27 \mathrm{~mW} / \mathrm{mm}^{2}$ ) intercalated by $11 \mathrm{~s}$ of darkness. Figure 6 shows examples of the photocurrents elicited by such protocol in HEK-293-transfected cells recorded at $-60 \mathrm{mV}$. In general, photocurrents from channelrhodopsins typically exhibited a peak current which relaxes to a steady-state component due to the desensitization of a fraction of the channels through equilibration of two open states and accumulation of nonconducting intermediates (Bamann et al., 2008; Berndt et al., 2010; Nagel et al., 2003). 


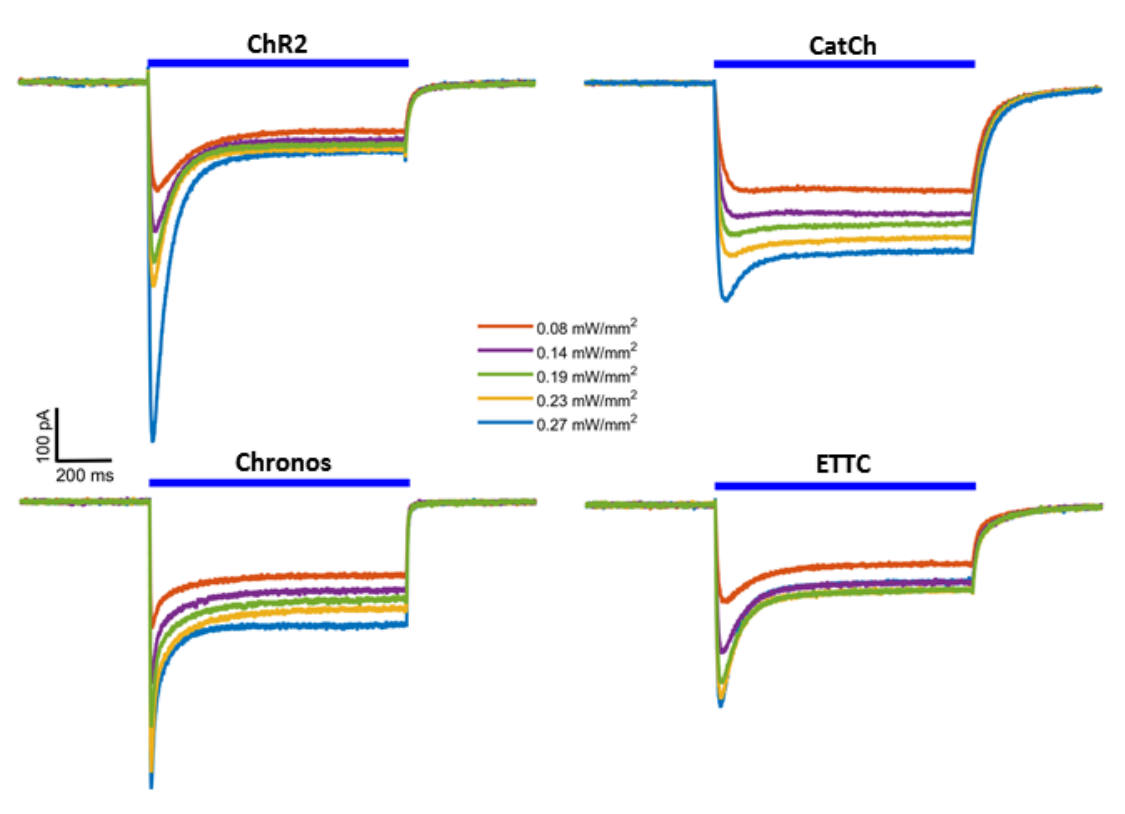

Figure 6. Example light-responses of the four light-sensitive channels tested. Channelrhodopsin-2 (ChR2; Nagel et al., 2003), CatCh (Kleinlogel et al., 2011), chronos (Klapoetke et al., 2014), and the ChETA variant E159T/T159C (ETTC; Berndt et al., 2011), were stimulated with a series of 1-s-long light-pulse (blue line above cell responses) of different intensities (colored traces).

As a matter of fact, at the highest light intensity tested $\left(0.27 \mathrm{~mW} / \mathrm{mm}^{2}\right)$, chronos exhibited the greatest average $( \pm S E)$ peak value $(479.5 \pm 48.5 \mathrm{pA}, \mathrm{n}=9$; Figure $7 \mathrm{~A})$, while all other channels' peak current were about $20 \%$ smaller (ChR2: $372.5 \pm 85.3 \mathrm{pA}, \mathrm{n}=6$; CatCh: $371.6 \pm 29.9 \mathrm{pA}, \mathrm{n}=4$; ETTC: $365 \pm 94.0 \mathrm{pA}, \mathrm{n}=5$ ). The steady-state component (Figure 7B) at the maximum light-intensity was greatest in CatCh $(259.8 \pm 31.6 \mathrm{pA}, \mathrm{n}=4)$, followed by chronos $(204.0 \pm 21.3 \mathrm{pA}, \mathrm{n}=9)$, ETTC $(162.9 \pm 48.2 \mathrm{pA}, \mathrm{n}=4)$, and ChR2 (82.9 $\pm 16.4 \mathrm{pA}, \mathrm{n}=$ 6). A direct comparison between the current responses under the expression conditions used is not adequate, as the expression of the different channelrhodopsins was driven by different promoters. In this set of experiments, however, the peak-to-steady-state current ratio does indicate an important property for some applications. In order to obtain a constant illumination, channels exhibiting peak-to-steady-state ratios close to 1 (indicating less desensitization) are favorable over channels with greater values. While all other channels exhibited light-intensity-dependent ratio (Figure 7C), CatCh's ratio was weakly lightdependent and was the smallest of all (at maximum intensity, $1.4 \pm 0.06, \mathrm{n}=4$; ChR2: $4.4 \pm$ $0.3, \mathrm{n}=6$; chronos: $2.4 \pm 0.06, \mathrm{n}=9$; ETTC: $2.6 \pm 0.1, \mathrm{n}=4$ ). 

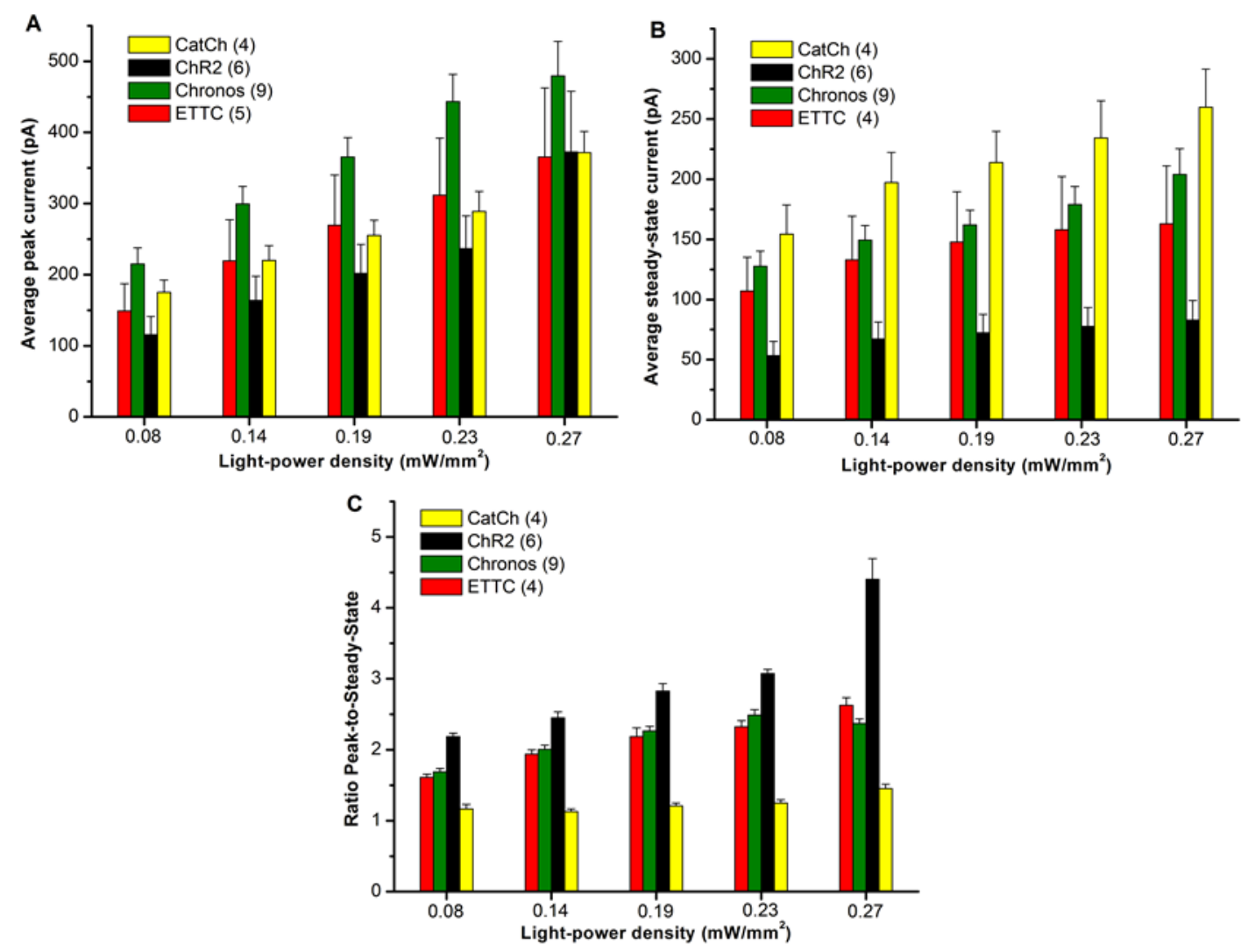

Figure 7. General properties of the photocurrents of the different light-sensitive channels tested. (A) Average peak current. (B) Average steady-state current. (C) Ratio peakto-steady-state. Numbers in parenthesis indicate number of cells recorded. Mean \pm standard error of the mean.

In order to quantify the onset/offset rapidness of the photocurrents, which are crucial for dynamic stimulation, I estimated their time-constants by fitting a single exponential to the onset/offset currents, from stimulus onset/offset until the peak/steady-state current respectively. Examples of the normalized on/off sample currents used for quantifying the kinetics of each channel can be seen in Figure 8A and B, respectively. Single exponential fittings to these currents revealed that chronos' performance is much superior to that of the other channels, for both, activation and inactivation (Figure $\mathbf{8 C}$ and $\mathbf{D}$, respectively). At the maximum intensity tested, chronos was about three times faster than channelrhodopsin-2 during activation and inactivation $\left(\tau_{\mathrm{on}}: 1.5 \pm 0.08 \mathrm{~ms}, \mathrm{n}=21\right.$ for chronos vs $4.9 \pm 0.32 \mathrm{~ms}, \mathrm{n}=$ 6 for ChR2; $\tau_{\text {off: }} 3.0 \pm 0.2 \mathrm{~ms}, \mathrm{n}=21$ for chronos vs $9.4 \pm 1.1 \mathrm{~ms}, \mathrm{n}=6$ for ChR2; mean \pm SEM. $\mathrm{t}$ test result: ${ }^{* * *} \mathrm{p}<0.0001$ for both, activation and deactivation). While it has been reported that the ETTC variant has improved kinetics over channelrhodopsin-2 (Berndt et al., 2011), at the light regime here tested, ETTC activation was significantly slower than ChR2 ( $\tau_{\text {on }}: 6.1 \pm$ 0.3, $\mathrm{n}=6$ for ETTC vs $4.9 \pm 0.32, \mathrm{n}=6$ for ChR2; $\tau_{\text {off: }} 10.4 \pm 0.7 \mathrm{~ms}, \mathrm{n}=6$ for ETTC vs $9.4 \pm 1.1$ 
ms, $n=6$ for ChR2; mean \pm SEM. t-test result: ${ }^{*} p=0.015$, for $\tau_{\text {on }}$ and $p=0.39$ for $\tau_{\text {off). CatCh }}$ showed the poorest performance of all.

A

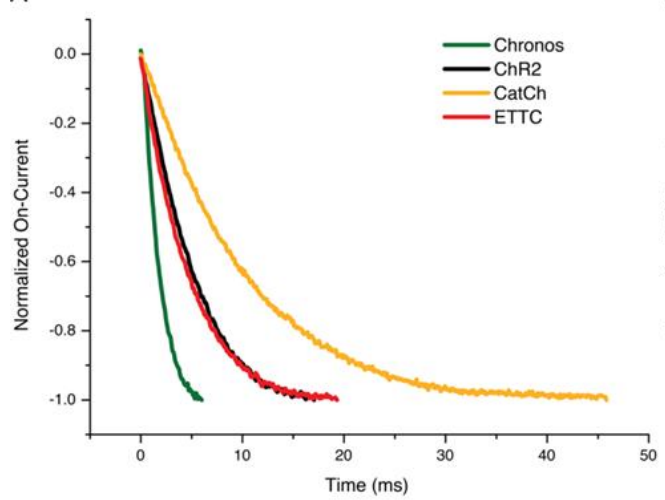

C

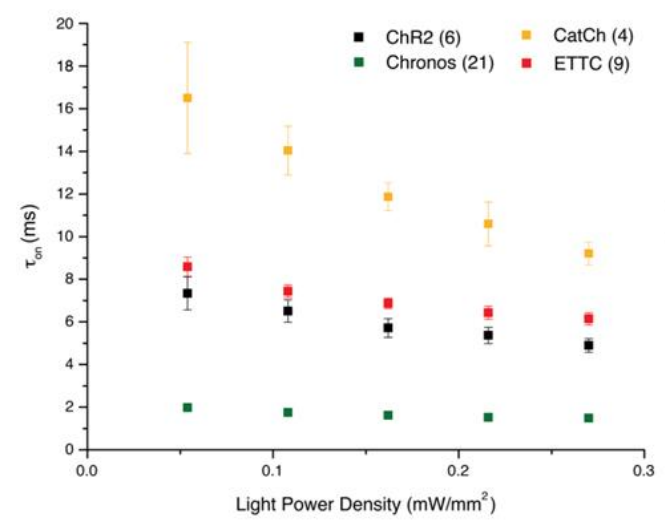

B

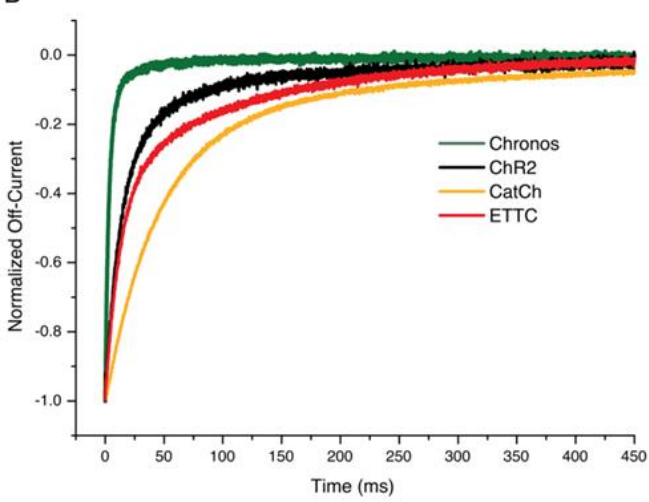

D

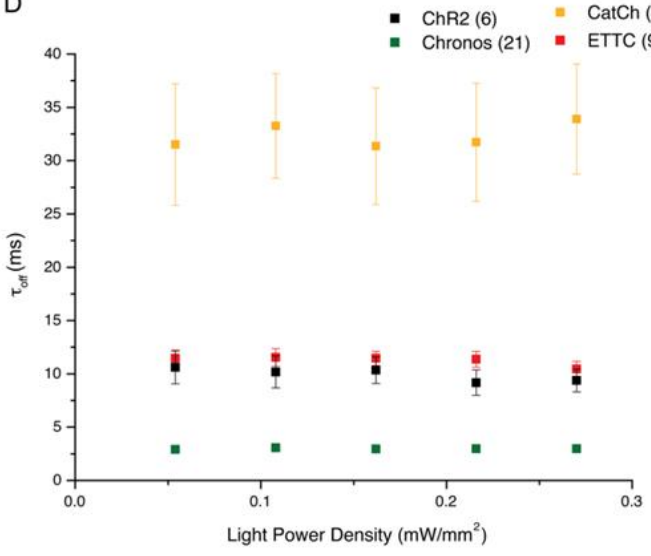

Figure 8. Photocurrent onset/offset kinetics. Example of normalized current onset (A) and (B) offset. Currents were normalized by peak amplitude (onset) and steady-state value (offset). Curves were then fitted with single-exponentials and time-constants plotted as a function of light intensity (C and D). (C) Time-constants for current onset. (D) Time-constants for current offset. Numbers in parenthesis indicate number of cells recorded. Mean \pm standard error of the mean.

From the first results with the light steps, it became clear that chronos was the very best candidate for applications involving dynamic photostimulation due to the very fast onset/offset of its currents. It has been reported that channelrhodopsins exhibit significant temperature dependence (Williams et al., 2013). For this reason, I decided to test whether increasing the recording temperature to values close to rodent's physiological temperatures improves chronos' activation/inactivation kinetics even further. Chronos-transfected HEK293 cells were submitted to the same experiment reported above, but at $36 \pm 1^{\circ} \mathrm{C}$ (Figure 9). 

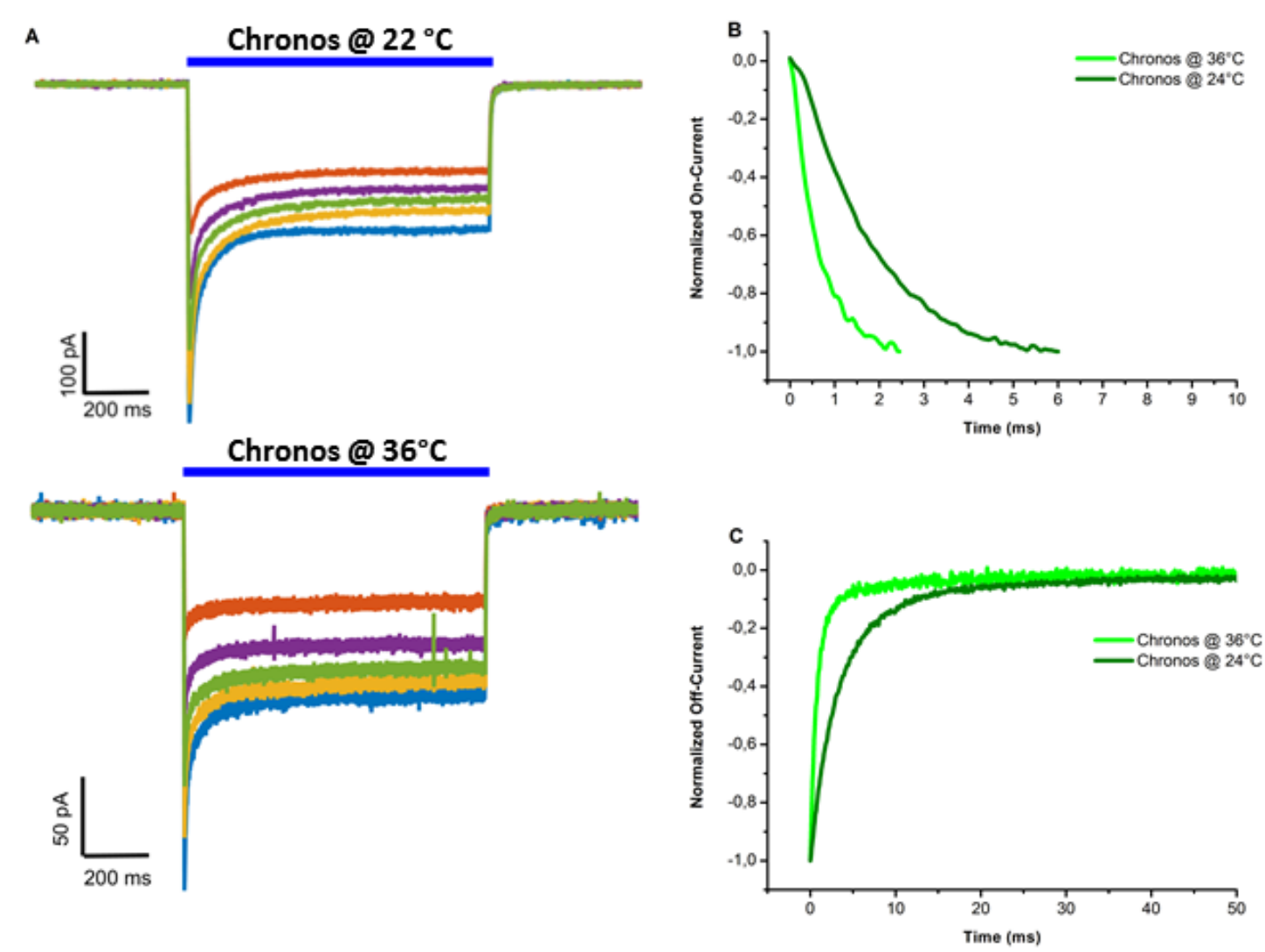

Figure 9. Effect of temperature on chronos' photocurrents. (A) Sample traces of responses to light-steps of different intensities (blue bar). (B, C) Examples of normalized current onset (B) and offset (C) from chronos-transfected cells at room temperature (dark green) and $\sim 36^{\circ} \mathrm{C}$ (light green).

At such temperatures, chronos' performance exhibited a dramatic 3-fold improvement over room-temperature recordings, with onset/offset time-constants at maximum intensity equal to $0.58 \pm 0.02 \mathrm{~ms}(\mathrm{n}=5$; vs $1.49 \pm 0.08 \mathrm{~ms}$ at room-temperature; Figure 10A) and $0.76 \pm 0.05 \mathrm{~ms}(\mathrm{n}=5$; vs $2.97 \pm 0.22 \mathrm{~ms}$ at room temperature; Figure 10B), respectively. The estimate $Q_{10}$ values for $0.08 \mathrm{~mW} / \mathrm{mm}^{2}$ and $0.27 \mathrm{~mW} / \mathrm{mm}^{2}$ are repecti elry 2.4 and 2.1 for onset time-constants and 2.9 at both light powers for offset.

In face of the clear superiority of chronos in terms of kinetics, and the fact neither ETTC nor CatCh were superior to the already-characterized channelrhodopsin-2 (Neef et al., 2013), I performed other experiments in order to further characterize chronos. Another method to evaluate kinetics performance is to analyze the frequency-response of the channels. To this end, I used a different light-stimulation protocol, consisting of a 10-s long light chirp from 0.1 to $100 \mathrm{~Hz}$ and maximum amplitude equals to $0.27 \mathrm{~mW} / \mathrm{mm}^{2}$ (Figure 11). As a reference, ChR2 was also tested in the same way. From the sample traces in figure 11, the difference in performance between chronos and ChR2 becomes clear as the stimulus frequency increases. 
A

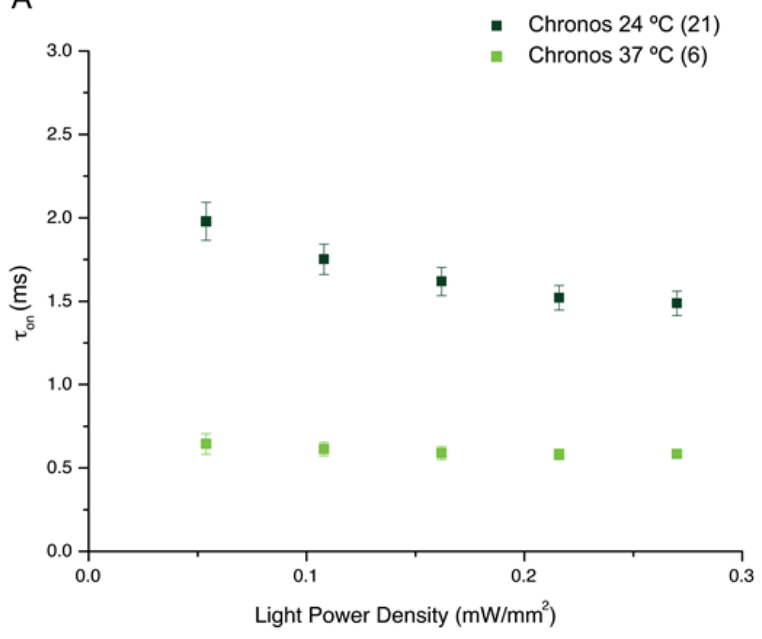

B

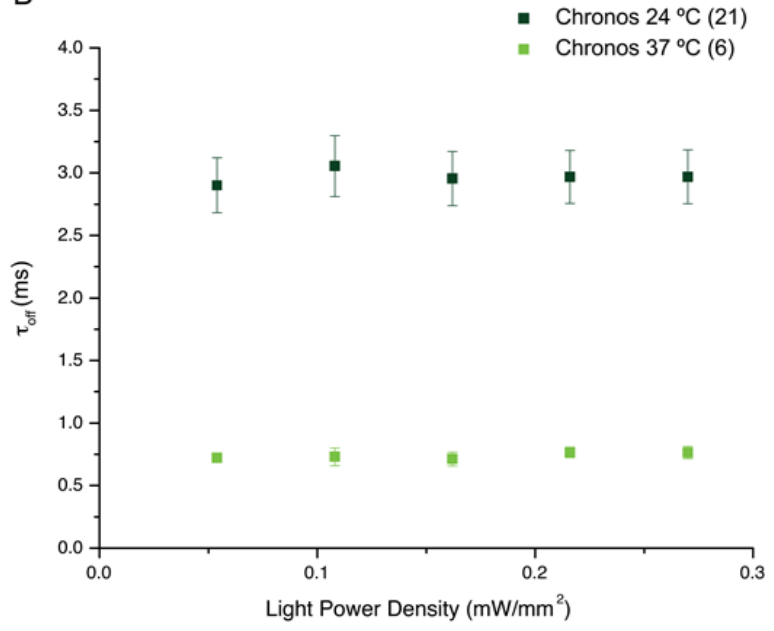

Figure 10. Quantification of activation (A) and deactivation (B) time-constants for chronos at different temperatures. Numbers in parenthesis indicate number of cells recorded. Mean \pm standard error of the mean.

Up to $2 \mathrm{~s}$ in the stimulus (approximately $20 \mathrm{~Hz}$ ), both, ChR2's and chronos' photocurrents did not exhibit attenuation in their intensities (Figure 11). Beyond the 2-s mark, however, channelrhopsin-2 current amplitude quickly decreased, indicating that the channel was not reliably following the light stimulus any longer, while chronos' currents still exhibited close-to-maximum amplitudes. At the end of the stimulus, i.e., at $100 \mathrm{~Hz}$, it was clear, however, that both showed significant attenuation in the photocurrents. When I measured the responses of chronos at higher temperature, however, once more it became clear the excellent performance of this channel: in the tested frequency interval, 0.1 to 100 $\mathrm{Hz}$, only minimal attenuation in the photocurrents' amplitude was observed (Figure 11, lowest trace). 


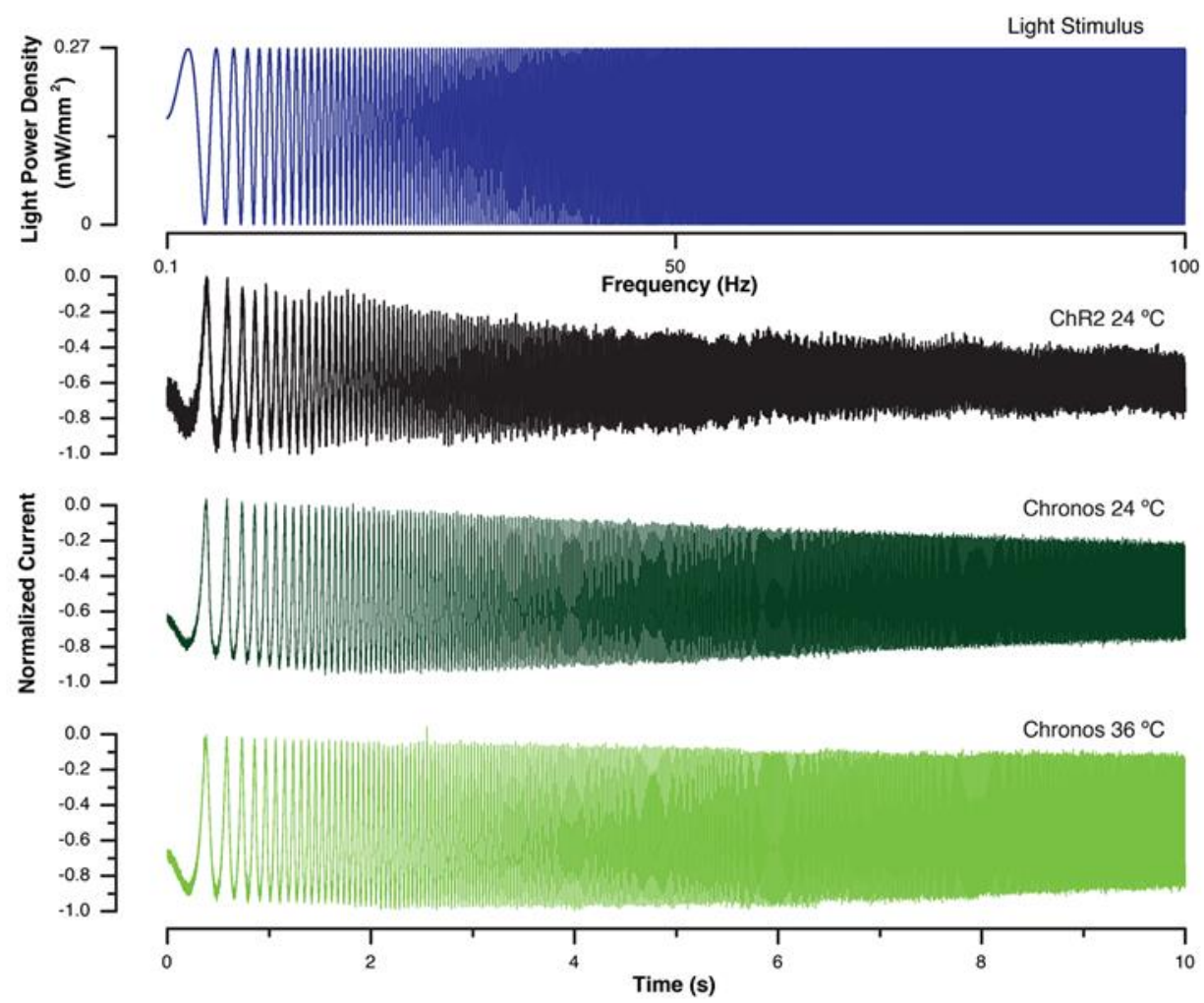

Figure 11. Responses to light chirps. Sample traces of photocurrents elicited by a light chirp from 0.1 to $100 \mathrm{~Hz}$ (blue). Channelrhodopsin-2 recorded at room temperature (black) exhibited strong reduction in the amplitude of the currents beyond the 2-s mark (approximately $20 \mathrm{~Hz}$ ). Chronos at room temperature (dark green) performed better that ChR2, but current amplitude still decreased significantly as the stimulus frequency increases. At $\sim 36{ }^{\circ} \mathrm{C}$ (light green), however, chronos was able to follow reliably stimulus in the range tested.

In order to quantify the results from the chirp protocol, I calculated the powerspectral density from the recorded currents and compared them to the typical responses of single-pole RC-type low-pass filters with various cutoff frequencies to voltage chirps with the same characteristics as the light (Figure 12). From this comparison, it is possible to observe that both, chronos and channelrhodopsin-2 behave as low-pass filters, "transmitting" better at lower frequencies. In addition, by "fitting" the photocurrents' spectra with the spectra of digital filters with different cutoff frequencies, one can describe the performance of the channels in terms of an equivalent cutoff frequency (defined as the frequency in which power drops to $1 / \sqrt{2}$ of the initial power): at room temperature, channelrhodopsin- 2 and chronos were equivalent to single-pole RC-filters with 25 and $57 \mathrm{~Hz}$, respectively. As observed in figure 11 , higher temperature improved chronos' performance, so that, at $\sim 36^{\circ} \mathrm{C}$, it resembles a filter with $130 \mathrm{~Hz}$ cutoff frequency. 


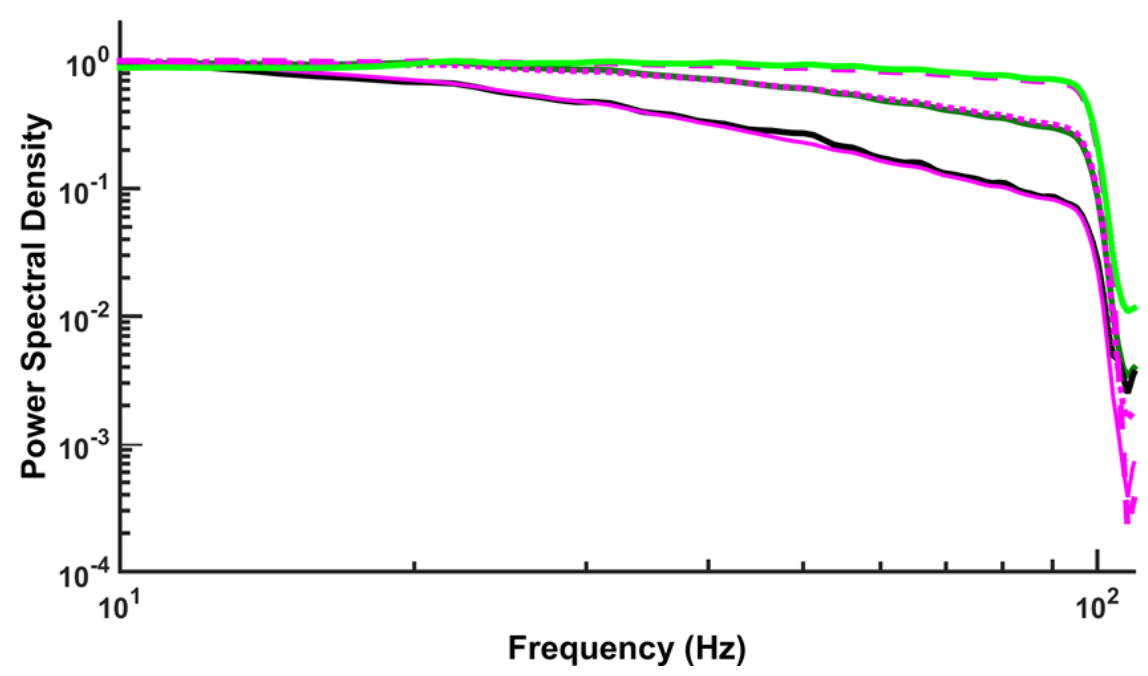

Figure 12. Power-spectral density of chirp responses. Channelrhodopsin- 2 at room temperature (black), chronos at room temperature (dark green), and chronos at $\sim 36{ }^{\circ} \mathrm{C}$ (light green) spectra can be approximated by single-pole low-pass filters (magenta) with $25 \mathrm{~Hz}$ (continuous), $60 \mathrm{~Hz}$ (dotted), and $130 \mathrm{~Hz}$ (dashed) cutoff frequencies, respectively. Chirp response spectra are the averages of 9 (black), 20 (dark green), and 5 (light green) recorded cells.

In terms of speed, chronos was without doubts the best candidate. Based on this, I performed other experiments aiming at testing whether the other two requirements, reproducibility and predictability, were also met by chronos.

\subsubsection{Reproducibility}

Reproducibility across cells was tested by using the same fluctuating light in different cells (Figure 13). Fluctuating stimulus with three correlation times $\left(\tau_{c o r r}: 1,5\right.$, and $50 \mathrm{~ms}$ ) were used. By calculating the Pearson correlation coefficient across cells of the normalized current responses for each of the correlation times, I was able to quantify the reproducibility of chronos' photocurrents. In Figure 13B, it is possible to see that for all correlation times tested, the reproducibility was high, as indicated by the average Pearson correlation coefficients always greater than 0.92 . One can also observe that the average correlation coefficient decreased as the stimulus correlation times decreased (faster fluctuations). 

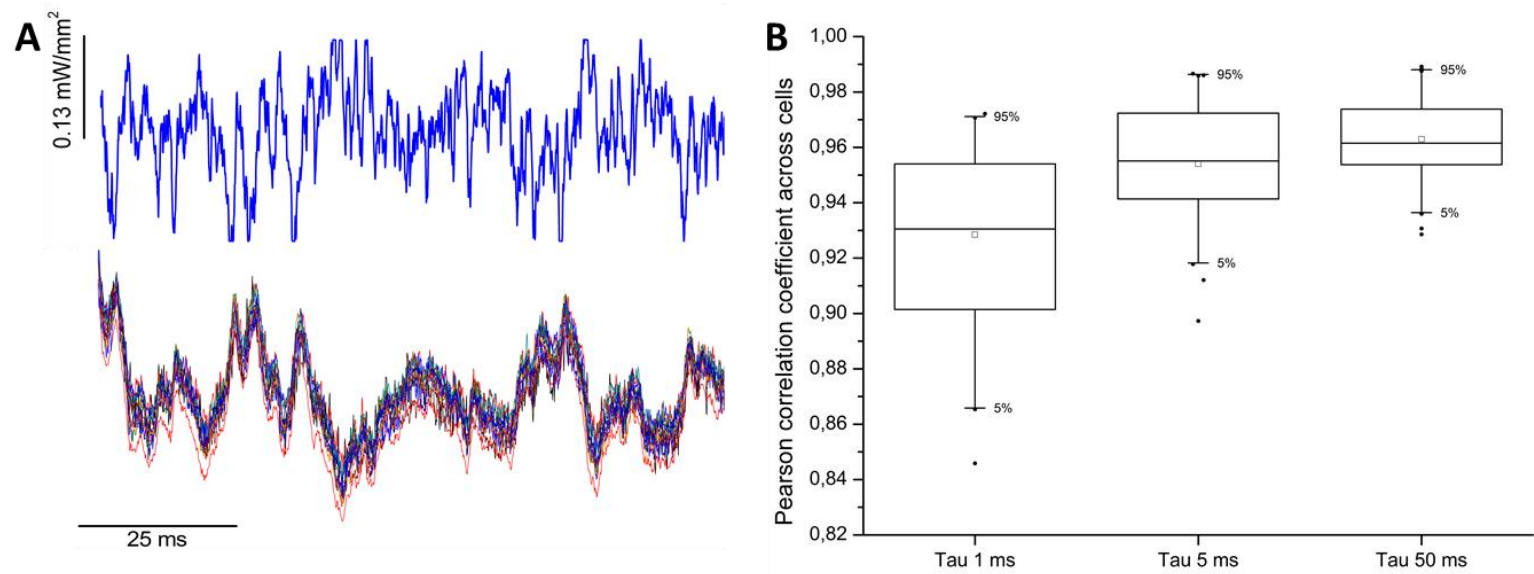

Figure 13. Chronos reproducibility test. (A) Sample of the light-stimulus used to measure reproducibility (upper blue trace; $\tau_{c o r r}=1 \mathrm{~ms}$ ) and superimposed recorded currents from 10 cells (lower traces). (B) Pearson correlation coefficient from 10, 11, and 11 cells tested with stimulus of, respectively, 1, 5, and $50 \mathrm{~ms}$ correlation time.

This drop in the correlation coefficient was particularly clear at $\tau_{c o r r}=1 \mathrm{~ms}$ and it probably reflects the fact that the channel was struggling with the fastest components in the stimulus. The high correlation coefficient nonetheless indicates that even for such an extreme condition as $1 \mathrm{~ms}$ correlation time, reproducibility was still very good. The averages \pm SEM were as follows: $\tau_{c o r r}=1$ ms: $0.93 \pm 0.03\left(\mathrm{n}=10\right.$ cells); $\tau_{\text {corr }}=5$ ms: $0.95 \pm 0.02(\mathrm{n}=11$ cells $) ; \tau_{\text {corr }}=50 \mathrm{~ms}: 0.96 \pm 0.02(\mathrm{n}=11$ cells $)$. At last, I tested how predictable chronos photocurrents were.

\subsubsection{Predictability}

Predictability is absolutely crucial in applications in which the precise stimulus waveform must be known, such as in the determination of the frequency-response function of neuronal populations (e.g., Higgs and Spain, 2009; Kondgen et al., 2008; Tchumatchenko et al., 2011). In order to test predictability, I used a linear systems approach: if channelrhodopsins behave linearly in the range of light intensity tested, by convolving their impulse-response function with the stimulus waveform, one finds the expected response (i.e., current) of the system. To do so, I first calculated chronos' impulse-response function (IRF) by taking the inverse Fourier transform of the ratio of the Fourier transforms of recorded photocurrents and the light-stimulus that elicited it (Figure 14). 

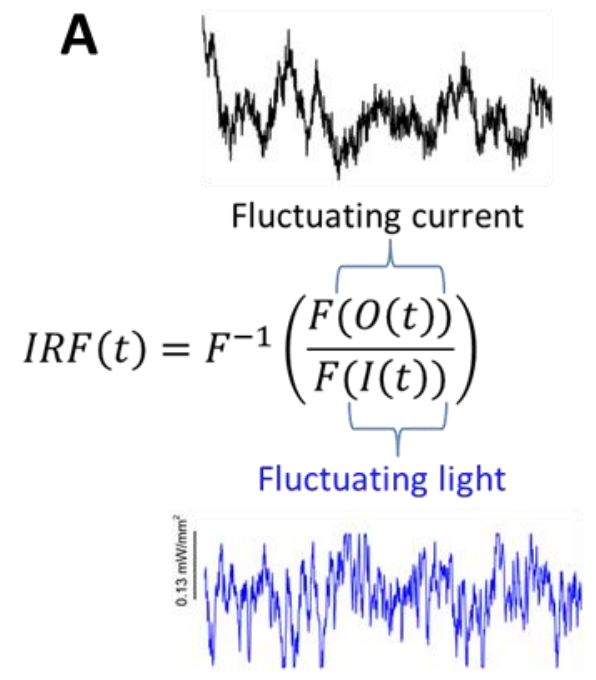

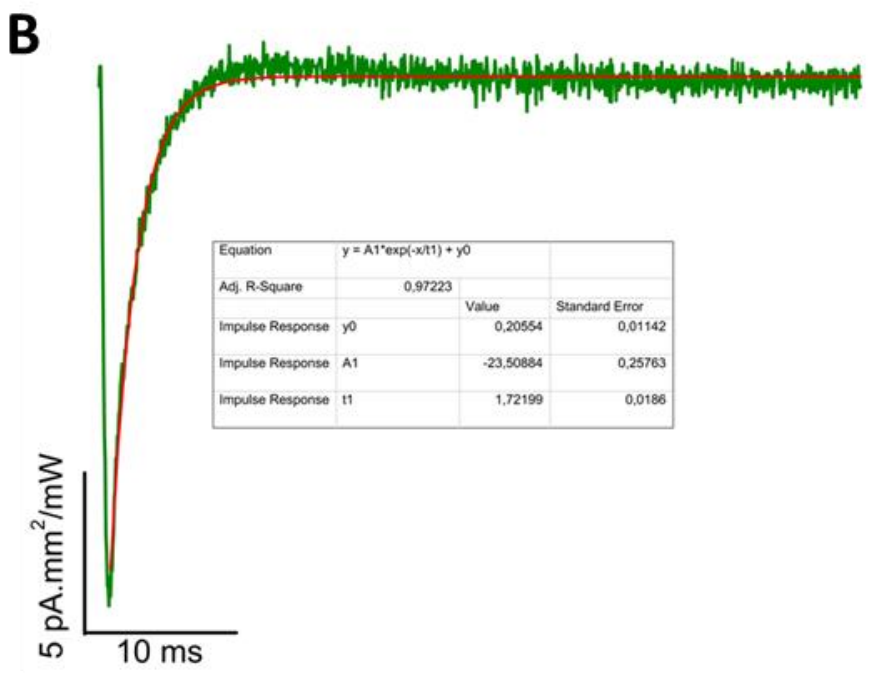

Figure 14. Calculation of the impulse-response function of chronos. (A) General procedure for calculating an impulse-response function. Basically, it involves taking the ratio of the Fourier transforms of the output (i.e., the recorded current, black trace) and the input (the light waveform, in blue), followed by the inverse Fourier transform of the result. (B) chronos' impulse response function.

The resulting IRF was then used in the convolution procedure to predict currents elicited by 6 different fluctuating stimuli, constructed using the same statistics but different random seeds. Prediction was quantified by determining the Pearson correlation coefficient between the predicted current and the actual recorded current elicited by stimulation with the fluctuating stimulus used in the prediction (Figure 15). The analysis of the correlation coefficients indicate that the currents can be predicted with high precision: $\tau_{c o r r}=1 \mathrm{~ms}, 0.99$ \pm 0.002 , and $\tau_{\text {corr }}=5 \mathrm{~ms}, 0.98 \pm 0.001$.

Altogether, the series of experiments here described showed that, with respect to speed, reproducibility, and predictability, chronos would be an excellent tool for CoDyPS. Since all the intended characterization was done, the next natural step was to test chronos in neurons.

\subsubsection{Tests in neurons: lack of neuronal expression}

From the first experiments with transfected HEK-293 cells, it had already become clear that chronos was potentially the best candidate as a tool to be used in CoDyPS. All subsequent experiments just confirmed this. 

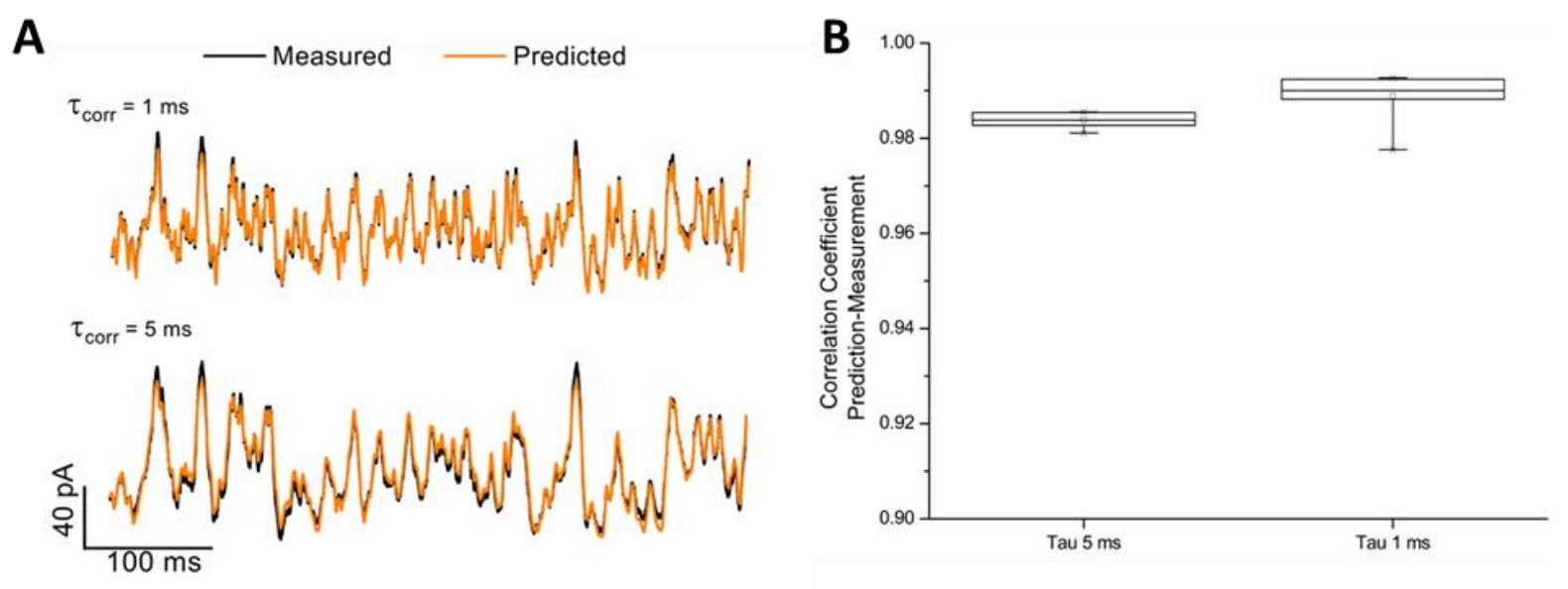

Figure 15. Prediction of chronos. By convolving the impulse response function with a stimulus waveform, one can predict the real current. (A) Comparison of measured current (black) and predicted current (orange) for two different input correlation times (1 ms, upper traces, and $5 \mathrm{~ms}$, lower traces). (B) Pearson correlation coefficients indicate the high predictability of the chronos' currents for these two input correlation times: $\tau_{c o r r}=1 \mathrm{~ms}$, $0.99 \pm 0.002$, and $\tau_{\text {corr }}=5 \mathrm{~ms}, 0.98 \pm 0.001$.

The last step involved the expression of chronos in neurons. Due to the fact that the ultimate goal for optimizing our dynamic photostimulation with chronos was to use it in experiments targeting cortical pyramidal neurons in acute brain slices, the method I chose to express chronos in neurons was viral transduction, with two parallel approaches. The first was direct viral infection of primary neuronal cultures, which would allow me a further characterization of chronos, and the second was in-utero intraventricular viral injection, a proxy to the final experiments I wanted to do. In order to target pyramidal neurons, chronos expression in the viruses was driven by the pyramidal neuron-specific $\mathrm{Ca}^{2+}-\mathrm{Calmodulin}$ Kinase II (CaMKII) promoter (Johansen et al., 2010; Wang et al., 2013).

Initially I tried to use an AAV1-CaMKII-chronos-EGFP virus in both, neuronal cultures and in-utero injections (see methods for details). In cultures, 1 to $2 \mu \mathrm{l}$ of viral suspension $\left(3.3 \times 10^{\wedge} 12 \mathrm{vg} / \mathrm{ml}\right)$ was added to each well of a 12-well-plate with 75,000 to 200,000 cells per well and 7 days in vitro. Expression was checked every day after infection. No fluorescence was detected even two weeks after infection. In in-vivo injections, approximately $1 \mu \mathrm{l} / \mathrm{embryo}$ of viral suspension was injected unilaterally in one of the lateral ventricles of E12.5 to E15.5 embryos. Three weeks after born, 60 - $\mu$ m-thick brain slices of PFA-perfused animals or 300$\mu \mathrm{m}$-thick acute brain slices were prepared. In both no sign of GFP fluorescence was detected. In addition, in acute slices I tried to detect light-responses using multielectrode extracellular recordings, with no responses being detected. Control in-utero injections with a control virus, 
AAV1-CaMKII-EGFP, however, did result in positive, widespread GFP fluorescence (Figure 16) suggesting that, for some reason, chronos was not being expressed.

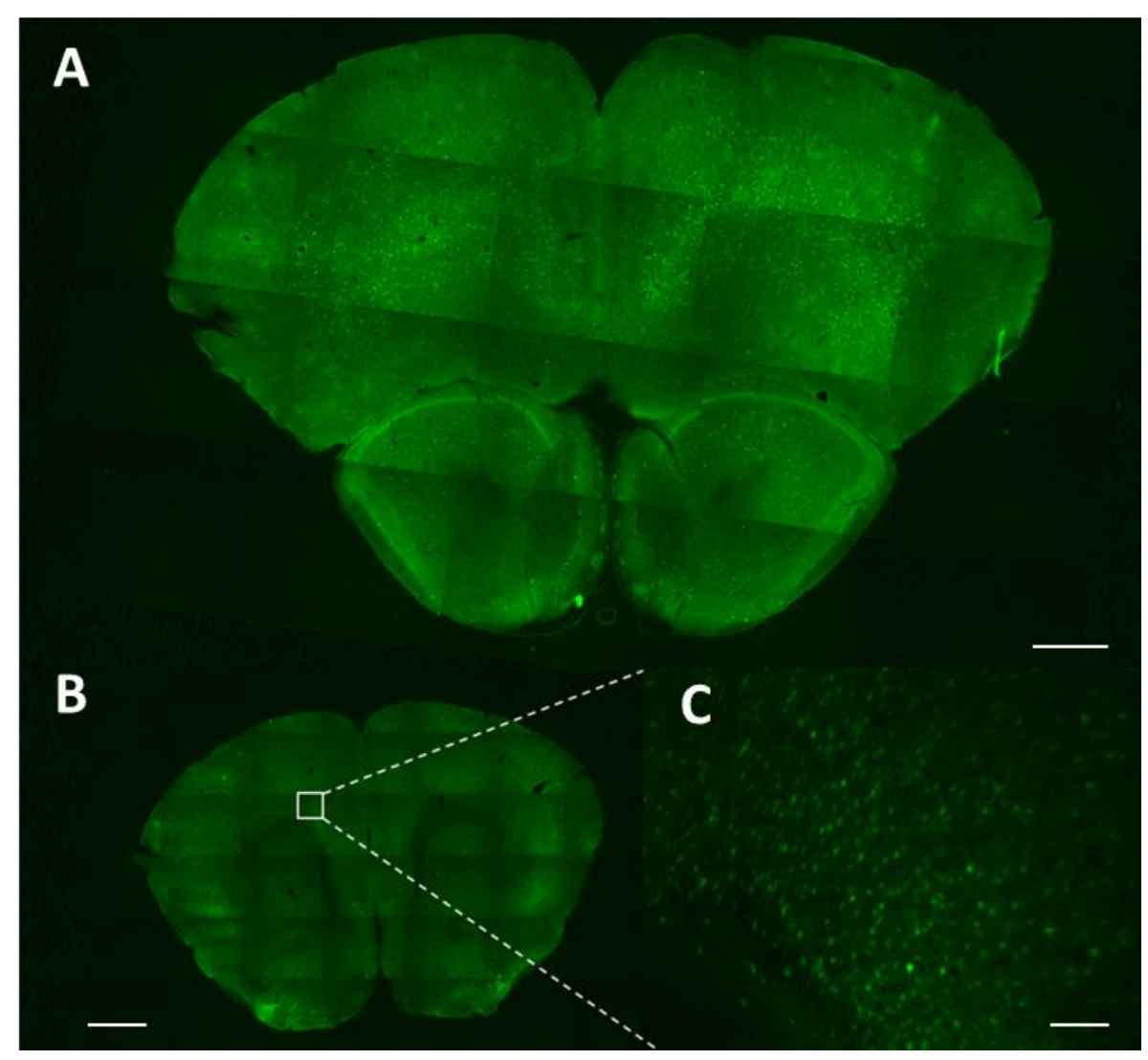

Figure 16. Sample photomicrographies of an AAV-CaMKII-eGFP in-utero injected animal. (A) In the cortex, eGFP-positive cells were predominately localized in deeper cortical layers. (B) As in A. (C) 20x magnification of the inset in B. Scale bars: (A) $500 \mu \mathrm{m}$, (B) $1 \mathrm{~mm}$, (C) $100 \mu \mathrm{m}$.

In my opinion, chronos sequence needs optimization or enhancing elements added to it, so that its performance can be totally appreciated in (mammalian) neurons. As this optimization was not the scope of my project, further characterization of our dynamic photostimulation paradigm needs either a better, optimized version of chronos, or another light-sensitive channel with similar performance to chronos, which, to the best of my knowledge, is not yet available. 


\subsection{Chapter 1: Discussion}

Light-sensitive ion channels offer important advantages over standard electrodebased stimulation techniques, the main ones being noninvasiveness and genetic selectivity (Boyden et al., 2005). As such, the development of the optogenetic toolbox widened the scope of experiments at reach of neuroscientists. Here, I sought to find an optimal optogenetic tool to be used for continuous dynamic photostimulation, or CoDyPS, a noninvasive optogenetic stimulation paradigm that allows the study of neuronal transfer functions in a much improved way, in comparison to standard, electrode-based methods.

\subsubsection{Advantages of using optogenetics for determining neuronal transfer functions}

As it will be explained in Chapter 2, the determination of neuronal transfer functions renders important evidences regarding information processing at both, neuronal and populational level (Higgs and Spain, 2009; Tchumatchenko et al., 2011). However, the standard methods employed for this, i.e., invasive, electrode-based techniques such as intracellular or patch-clamp recordings, are suboptimal, in that they are very much laborious and time-consuming; in order to properly characterize the gain function of a certain cell type, one must have about 2 to 3 hours of recordings (resulting in about 20000 to 30000 spikes, at 2-3 Hz firing frequency). Such long recordings are not possible to obtain from single cells, so multiple cells have to be recorded and pooled together. Therefore, with intracellular or patchclamp recordings it is not possible to obtain transfer function from single cells. By means of noninvasive stimulation with CoDyPS, associated with extracellular recordings, such long recordings from single cells become feasible, and data acquisition can be sped up significantly (Neef et al., 2013). By using multielectrodes, one can even record from multiple cells simultaneously, increasing even further the throughput of the method.

\subsubsection{Chronos performance}

From my characterization with the different light-sensitive ion channels it is clear that chronos had a much improved performance over the other tested candidates. Over the range of intensities tested, chronos' performance was about 2-3 times faster than channelrhodopsin-2, a value similar to what has been previously described (Klapoetke et al., 2014). The much faster performance of chronos is related to its high light sensitivity (Klapoetke et al., 2014). Its light sensitivity is also beneficial in experiments aimed at 
controlling two different neuronal populations by means of channelrhodopsins with spectrally-separated activation. Since all red-light activated channel known to date also exhibit a residual, blue-light sensitivity (Lin et al., 2013; Mattis et al., 2012; Prigge et al., 2012), the high-sensitivity of chronos allows for a much lower light-regime to be used, reducing the likelihood of activation of the red-sensitive channel (Klapoetke et al., 2014). With $\mathrm{Q}_{10}$ around 2-3, chronos current's onset/offset temperature dependences are in the same range as for channelrhodopsin-2 (Williams et al., 2013).

The fact that chronos' currents are also highly reproducible and predictable is likewise paramount to the intention of using it in CoDyPS. In Figure 15A, one can observe that the mismatch between predicted and measure currents happen mainly at the peaks. This suggests an escape from the linear regime, possibly due to the light intensity. Even though the predictability still reached very high coefficients, it might be helpful to increase this value even further, particularly at the peak currents, possibly by using linear-nonlinear models to predict currents. This might be necessary in face of the fact that, in order to measure neuronal transfer functions, one must know precisely the stimulus waveform triggering action potentials. Due to the fact that the reduction in prediction power happened at the peak current, i.e., at the current values most likely to elicit spikes, a further increase in the predictability particularly at peaks might be advisable.

While in terms of its biophysical properties and linearity chronos is a fantastic channel, the main issue is obviously the lack of expression in neurons. Even though I used different approaches for obtaining satisfactory chronos expression in different neuronal models, none succeeded. The $\mathrm{Ca}^{2+-}$-calmodulin kinase II (CaMKII) promoter is known to be widely expressed in the cortex, and control experiments using AAV-CaMKII-EGFP vectors did result in EGFP expression, indicating that both, the AAV vector itself and the CaMKII promoter were not an issue, as found in the literature (Wang et al., 2013; Watakabe et al., 2015). In the work describing chronos, Klapoetke et al (2014) use Ca ${ }^{2+}$-phosphate transfection in neuronal cultures and in-utero electroporation for slice electrophysiology. In both, a CAG promoter, known as a very strong promoter, was used. In preliminary work not described here, I was not able to detect CaMKII-driven chronos expression in in-utero electroporated animals either. Probably due to the phylogenetical distance between Alga and Metazoa, one could expect that the expression of certain proteins from one group to the other is not trivial, due to distinct cellular mechanisms involved in transcription, translation, and protein trafficking. In fact, it is known that the archael chloride pump halorhodopsin displays impaired membrane trafficking when expressed in high levels in mammalian cells (Gradinaru et al., 2008). A way to circumvent this problem is to optimize the microbial opsin genes to 
metazoan systems by the insertion of metazoan signaling motifs, such as membrane, endoplasmic reticulum, or Golgi export tags (Gradinaru et al., 2010).

Altogether, it seems to me that an optimization of chronos construct has to be done so that it can be used as a tool for controlling the activity of pyramidal neurons (which, up to date, requires the use of the CaMKII promoter). Such optimization may be necessary, both, at chronos' sequence level or by using different enhancers/regulatory sequences that optimize translation, translation and/or protein trafficking. 



\section{Chapter 2: The gain function in electrically defined interneuronal populations}

\subsection{Introduction}

"In recognition of their work on the structure of the nervous system". So was justified the 1906 Nobel Prize in Physiology or Medicine shared between Camillo Golgi and Santiago Rámon y Cajal. Rámon y Cajal is today one of the most cited classical scientists in history (Lopez-Munoz et al., 2006). His neuron doctrine, which states that nerve cells exhibit a relation of contiguity, rather than continuity as so believed, is considered a milestone of modern neuroscience (Lopez-Munoz et al., 2006). In the same year, the English physiologist Charles Scott Sherrington published "The Integrative Action of the Nervous System", laying the foundations of modern neurophysiology (Sherrington, 1906). Ever since, two of the main tasks for neuroscientists have been the description of the multitude of neuronal types in the brain and the characterization of their functions.

In the cerebral cortex two main types of neurons coexist. Pyramidal neurons, or principal cells, are excitatory (glutamatergic) nerve cells with relative stereotypical morphology and physiology, which comprise approximately $80 \%$ of the neocortical neurons (DeFelipe, 1993; DeFelipe and Farinas, 1992). Being excitatory, they are responsible for transmission of information to, within, and from the cortex. The remaining ca. $20 \%$ are interneurons, mostly inhibitory cells which exhibit enormous morphological, molecular and physiological diversity (Markram et al., 2004). Also referred to as "local circuit neurons", these cells are mainly responsible for the modulation of information in their circuits.

\subsubsection{The Neocortical Inhibitory System}

Although extremely diverse, interneurons possess a few features that are common to the vast majority of these cells - but not all of them - and distinguish them from pyramidal cells: their main neurotransmitter is GABA (thus, making them inhibitory cells), most mature interneurons exhibit aspiny dendrites, and their axons typically arborize within cortical columns and rarely target distant brain areas (Markram et al., 2004). Aside these few properties, interneurons can diverge in nearly every aspect of their phenotypes: morphology, 
physiology, neurochemistry and postsynaptic targets (Ascoli et al., 2008; Markram et al., 2004). This multimodal source of variety has turned the classification of interneurons into classes a major concern to the scientific community (Ascoli et al., 2008; Druckmann et al., 2013; Gupta et al., 2000; Markram et al., 2004). Despite the fact that some theories propose that well separated types of interneurons do not exist (Battaglia et al., 2013), most of the community currently accepts the existence of distinct interneuronal classes (Ascoli et al., 2008). While a complete description of all interneuronal classes is not the scope of this section, the description of a few of the most common interneuronal types is an interesting way to contemplate the cellular variety among these cells.

The main specifier of interneuronal classes is their morphology, with often the very names of the main classes alluding to their appearance. The most frequent interneuron, comprising nearly $50 \%$ of the cells, is the basket cell (Markram et al., 2004). Its name comes from the basket-like appearance of its axonal trees around the somata of pyramidal neurons, which indicates a fundamental characteristic of this class: they target only the soma and proximal dendrites of the principal neurons (Druga, 2009; Jiang et al., 2015; Kawaguchi and Kubota, 1997; Kubota, 2014). With respect to their electrophysiological profiles, these cells typically emit fast spikes with a pronounced fast afterhyperpolarization. Upon sustained stimulation, high frequencies are reached and little to no spike adaption is seen (Thomson and Lamy, 2007). Chandelier or axo-axonic cells also get their names from the appearance of their axonal arbors, which exhibit parallel cartridges resembling candlesticks in a chandelier (Woodruff et al., 2010; Woodruff et al., 2011). These cells exhibit high specialization regarding postsynaptic targeting, with axon terminals establishing synapses only with the axon initial segment of pyramidal neurons (Kawaguchi and Kubota, 1997; Kubota, 2014). Similar to basket cells, these are also capable of emitting high-frequency spikes trains, though not as fast as the basket cells. The firing pattern similar to that of chandelier and basket cells is referred to as fast spiking (Ascoli et al., 2008). Martinotti cells typically display a long ascending axonal tree with further lateral arborization in cortical layer I (Kawaguchi and Kubota, 1997; Kubota, 2014). They target mainly layer I apical dendrites of pyramidal cells and, differently from basket and chandelier cells, shows strong spike adaptation upon stimulation (Wang et al., 2004). Bipolar cells form a population of interneurons whose main targets are other interneurons (Jiang et al., 2015). These cells exhibit a variety of firing patterns, such as regular, irregular, bursting, and adapting spikes (Cauli et al., 2014; Jiang et al., 2015).

Regarding interneuronal firing patterns, it is known that these cells exhibit a rather stereotyped collection of patterns in response to suprathreshold current steps. A unified 
nomenclature has been used to classify and describe each of these patterns (Ascoli et al., 2008). Basically, two aspects of the response to depolarizing suprathreshold currents must be taken into account: the response at the onset of the stimulus and the "steady-state" response, i.e., the firing pattern after stimulus onset. Onset response can be classified as "burst", "delayed" and "continuous", while steady-state response can be "fast spiking", "nonadapting non-fast spiking", "adapting", "irregular spiking", "intrinsic burst firing", and "accelerating" (Ascoli et al., 2008; Druckmann et al., 2013). Within the fast spiking subset, interneurons often exhibit a "stuttering" pattern, which is distinct from the burst, continuous, and delayed patterns (Ascoli et al., 2008; Druckmann et al., 2013).

With respect to neurochemical markers, calcium-binding proteins and neuropeptides have been used to distinguish cortical interneurons (Ascoli et al., 2008; Cauli et al., 2014; Kawaguchi and Kubota, 1997; Markram et al., 2004). Of particular interest, the calciumbinding protein parvalbulmin (PV) and the neuropeptide somatostatin (SST) have been show to label non-overlapping groups of interneurons in the cortex (Ascoli et al., 2008; Kawaguchi and Kubota, 1997). Later, another marker, the ionotropic serotonin receptor 5HT3a (5HT3aR), was shown to label a third, distinct population, and that, together with parvalbulmin and somatostatin, these three markers covered in a non-overlapping manner nearly $100 \%$ of the interneurons (Figure 17; Rudy et al., 2011).

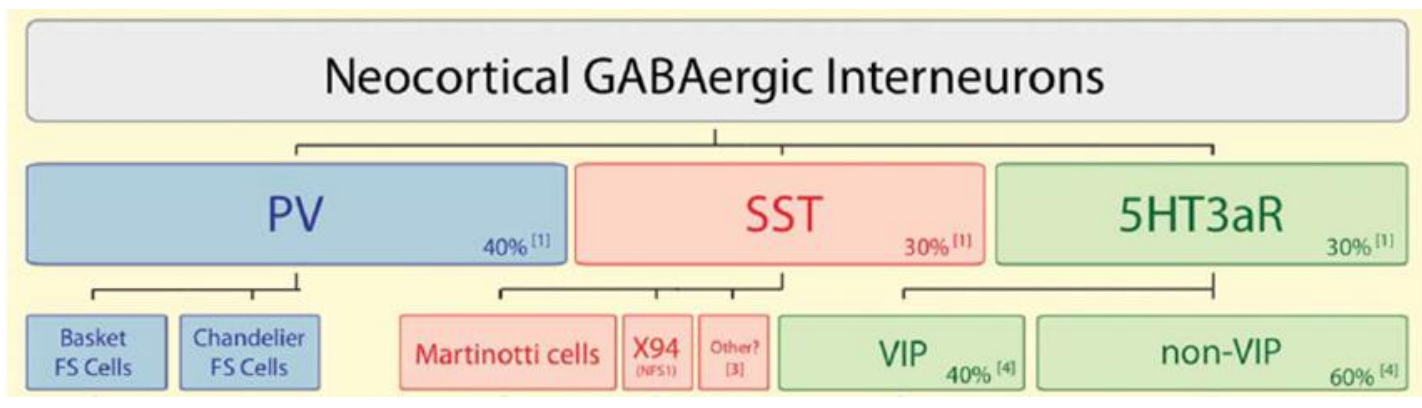

Figure 17. The classification of different interneuronal classes with respect to neurochemical markers. Parvalbulmin (PV), somatostatin (SST), and the ionotropic serotonin receptor 5HT3a are specific markers of three non-overlapping groups of interneurons and account for $100 \%$ of the neocortical GABAergic neurons. Modified from Rudy et al (2011).

Thanks to such genetic markers, specific interneuronal populations can now be targeted to express genetically-encoded tools, such as channelrhodopsins or calcium indicators, and their particular functions are now being revealed (Atallah et al., 2012; Cardin et al., 2009; Gradinaru et al., 2007; Olsen et al., 2012; Pfeffer et al., 2013; Sohal et al., 2009).

$\mathrm{PV}^{+-}$-cells, which exhibit fast spiking firing patterns, such as basket and chandelier cells, have been associated with the emergence of oscillatory network behavior, particularly 
in the gamma frequency (30 - $100 \mathrm{~Hz}$ ) (Cardin et al., 2009; Sohal et al., 2009). While the involvement of other neocortical interneuronal classes in the generation of brain rhythms is not yet clear (Buzsaki and Wang, 2012), in the hippocampus, somatostatin-expressing interneurons exhibit resonance bands at theta frequencies (Pike et al., 2000). Most of the studies investigating the contribution of interneurons to brain rhythms, however, have focused on a noise-free strong oscillatory input drive to the neurons. However, it has been shown that the presence of background noise mimicking membrane potential fluctuations observed in-vivo is determinant to the characterization of the response of neuronal populations to inputs of various frequencies (Destexhe et al., 2001; Destexhe et al., 2003; Fourcaud-Trocme and Brunel, 2005; Fourcaud-Trocme et al., 2003; Kondgen et al., 2008; Tchumatchenko et al., 2011). This so-called frequency-response function of neurons is directly related to their ability to convey transient and oscillatory signals, and its understanding can contribute to the comprehension of specific functions executed by different neuronal classes, and of the underpinning mechanisms of brain oscillations.

\subsubsection{Neuronal dynamic gain functions}

Brain rhythms in the range of a few up to hundreds of Hertz have been recorded in multiple brain areas. In-vivo recordings, however, have shown that neurons fire at much lower frequencies than those reached during network oscillations (Buzsaki and Draguhn, 2004). The generation of such rhythms and the encoding thereof must, thus, happen at the populational level (Kondgen et al., 2008). It has been shown that the presence of input noise, such as the fluctuating membrane potential due to the barrage of synaptic inputs in-vivo (Destexhe et al., 2001; Destexhe et al., 2003; Pare et al., 1998), is fundamental for a proper characterization of population dynamics. Such findings were first brought about by theoretical studies showing that spiking neuronal models subjected to fluctuating noise are capable of following arbitrarily high input frequencies (Brunel et al., 2001). For that, Brunel et al (2001) used a simple neuronal model with an instantaneous spike generation mechanism. Later, by means of computational simulations using neuronal models with realistic spike generation features, it was shown that the maximum speed neurons can reliably track stimuli is set exactly by the rapidness of spike generation, rather than spike firing frequency or membrane time constant (Fourcaud-Trocme and Brunel, 2005; FourcaudTrocme et al., 2003; Naundorf et al., 2005). Experimental confirmation of the abilities of pyramidal neuron populations to track high-frequency stimuli was given by Kondgen et al (2008), which showed that layer V pyramidal neurons exhibit a low-pass band behavior and are capable of following stimuli of frequencies up to $200 \mathrm{~Hz}$. Later, Tchumatchenko et al 
(2011) confirmed once more, now in both, frequency and time domains, that neuronal populations are able to respond in an ultrafast manner to minimal input changes. Neuronal conductance-based models, which are equipped with Hodgkin-Huxley-based sodium and potassium conductances (Hodgkin and Huxley, 1952), however, failed to reproduce the steep onset initiation dynamics and the high-frequency cutoff observed in experiments (Naundorf et al., 2006). These observations posed the question of whether unknown biophysical properties related to action potential onset would not be the determinant of high cutoffs.

One of the proposed mechanisms involved $\mathrm{Na}^{+}$-channel cooperativity. In models exhibiting cooperativity, it was shown that both, onset steepness and high cutoff frequencies could be achieved (Naundorf et al., 2006; Wei and Wolf, 2011). In line with the models, it was shown that experimental manipulations that slow down action potential onset strongly reduce high-frequency encoding in pyramidal neurons (Ilin et al., 2013). Another proposed explanation involved the compartmentalization of the spike initiation site (the axon initial segment), and the soma. Based on this model, a geometric discontinuity between soma and axon initial segment causes $\mathrm{Na}^{+}$-channels to open in an all-or-none fashion, resulting in very steep spike initiation (Brette, 2013). Recently, it was proposed by means of theoretical and experimental work that the size of dendritic trees can also contribute to the steep spike onset and associated high-frequency gain (Eyal et al., 2014). Based on these models, one can predict that different interneuronal classes, which diverge particularly with respect to their dendritic arbors, should exhibit high-frequency gains correlated with their dendritic trees.

While all the aforementioned studies were performed in pyramidal neurons, the gain function of noise-driven interneurons is still unknown. Considering the importance of brain oscillations in a number of brain functions, and the strong link of interneurons in the genesis and modulation of these oscillations, the determination of the frequency-response function of interneurons is an essential step to elucidate both the functional role of distinct interneurons in terms of information processing, and to explain how oscillations in different frequency bands come about and interact.

\subsubsection{Goals in this project}

Here I sought to characterize how distinct interneuron types, as defined by their electrical profiles, encode information in the fluctuation-driven regime. This characterization was made by means of the calculation of the frequency-response, or dynamic gain function, of each of these different interneuron types. 


\subsection{Results}

As explained in the introduction of this chapter, neocortical GABAergic interneurons form an immensely diverse population of cells. This diversity can be contemplated by multiple aspects of their phenotypes, such as their morphology, postsynaptic target, neurochemical profile and electrophysiological characteristics, and strongly suggest that each of these interneuronal subpopulations perform different tasks within their circuits. Such circuit specializations would also suggest that the way each of these interneuronal subtypes encode information might differ. In this section, I describe the results from the determination of the frequency-response function, or dynamic gain function, of different interneurons, as defined by their electrical types (e-types). The characterization of the gain function of these cells sheds light onto their functions within circuits. For that I used whole-cell current-clamp recordings in frontal cortical interneurons (motor and prefrontal cortices) of two different mouse lines: PVAI32 and NKTDTO. In the first line, parvalbulmin-positive interneurons express channelrhodopsin-2 fused to a green fluorescent protein. No optogenetic stimulation was performed. NKTDTO animals express red fluorescent protein under an inducible CREand NKX2.1 promoter-dependent way. The NKX2.1 promoter is specific to medial ganglionic eminence-derived interneurons (for details, see Methods). Recorded interneurons were first classified in different electrical types, as suggested in the literature (Ascoli et al., 2008; Druckmann et al., 2013), and then their gain functions were calculated.

\subsubsection{Determination of the electrical type of interneurons}

With respect to their electrophysiological profiles, interneurons often exhibit stereotyped responses to suprathreshold current steps. These responses have been categorized into so-called electrical types (e-types) and a standard nomenclature to each type has been suggested (Ascoli et al., 2008; Druckmann et al., 2013). Here, I classified neurons following the recommended guidelines (Ascoli et al., 2008). As the amount of current required for inducing firing in neurons depends heavily on biophysical parameters such as cell capacitance and membrane resistance, which may vary significantly across different cells and cells classes, it is not possible to establish an absolute current level across all cells for etype categorization (400 pA current injection in one cell might be just enough to reach threshold, while in others it can be enough to induce firing rate saturation). In order to avoid such issues, I opted to perform the e-type categorization at two relative levels: the rheobase, i.e., the minimum current required to induce spikes, and $1.5 \mathrm{x}$ the rheobase current. For that, 
cells were stimulated with increasing 15 pA current steps of 500 ms until rheobase and $1.5 \mathrm{x}$ rheobase levels were identified.

Six main electrical types were found (Figure 18), and five of these were classified as suggested by Ascoli et al (2008): continuous adapting (CA; Figure 18A), continuous fast spiking (cFS; Figure 18B), delayed fast spiking (dFS; Figure 18C), stuttering fast spiking (sFS; Figure 18D), and non-adapting non-fast spiking (NANFS; Figure 18E).

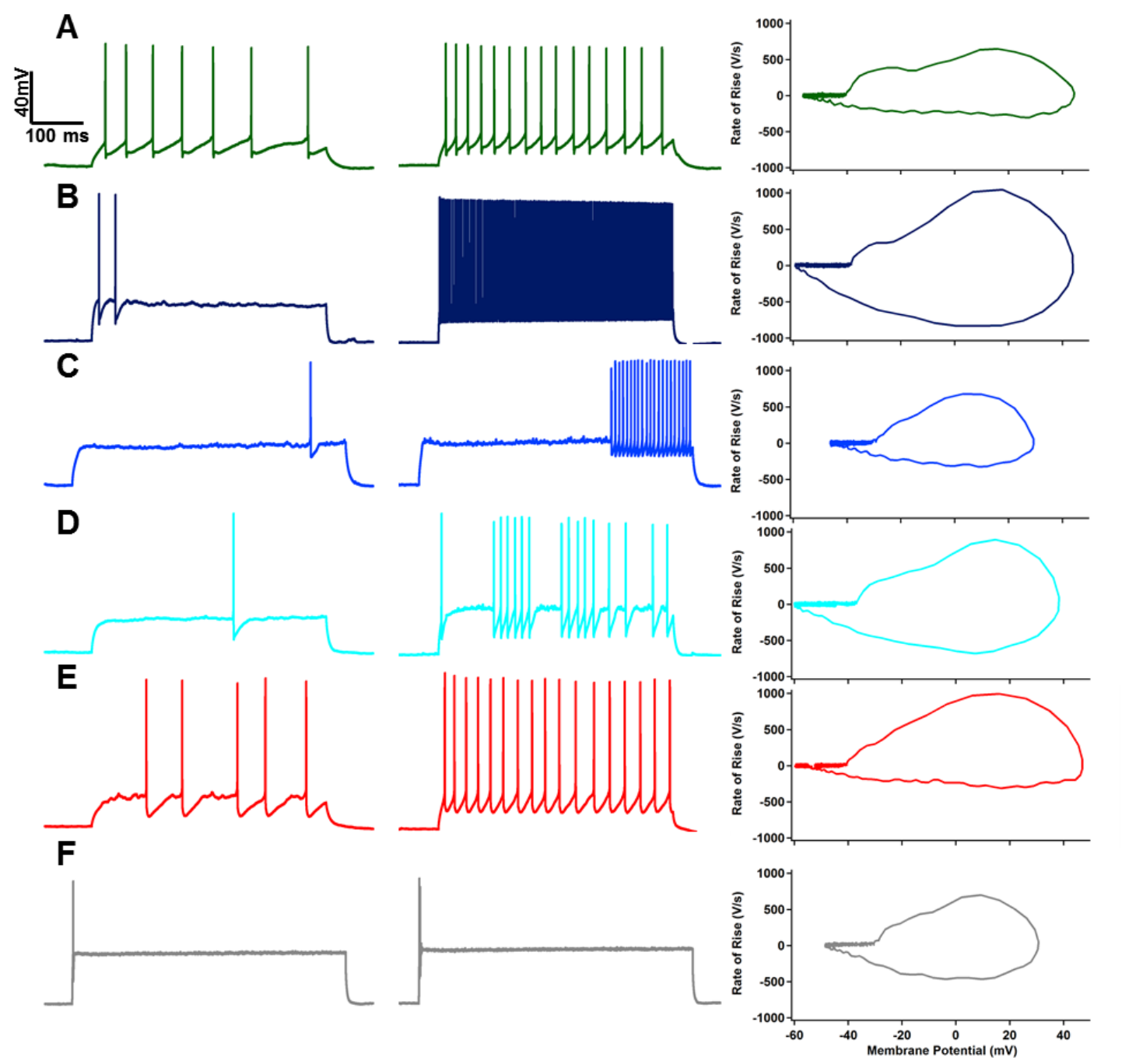

Figure 18. Examples of the six interneuronal e-types found. (A) Continuous adapting (CA), (B) Continuous fast spiking (cFS), (C) delayed fast spiking (dFS), (D) stuttering fast spiking (sFS), (E) Non-adapting non-fast spiking (NANFS), and (F) Single spikers (SSP). Left: examples of the response to a 500-ms depolarizing step at the rheobase current-level; middle: response at $1.5 \mathrm{x}$ rheobase current-level. Right: phase plots $(\mathrm{dV} / \mathrm{dt}$ vs membrane potential) of the first spike elicited at rheobase. Observe the big variance with respect to the hyperpolarizing phase of the action potential (negative values of rate of rise).

One class is not contemplated by the nomenclature in Ascoli et al. These cells often exhibited a single spike at the onset of the pulse for current values exceeding 2-3 times rheobase (eventually a second spike at the onset could also be seen). I refer to these cells as 
single-spikers (SSP; Figure 18F). For most of the analysis I grouped all three subtypes of fast spiking neurons into a single group called fast spikers (FS). Figure 19 summarizes the number of cells per e-type (Figure 19A) or per fast spiking subtype (Figure 19B).
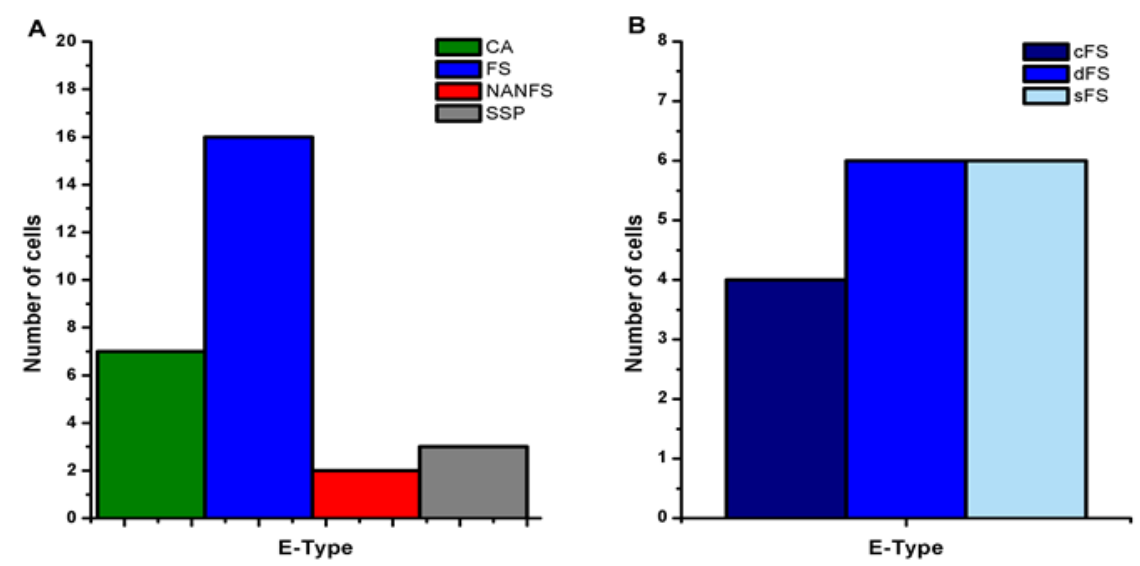

Figure 19. Number of cells per e-type (A) or per fast spiking subtype (B). (A) With respect to the steady-state categories (burst, continuous or delayed), only the continuous subtype was found for adapting (green; CA) and non-adapting non-fast spiking cells (red; NANFS). Fast spiking subtypes (blue; FS) were grouped together. Three cells exhibited a particular behavior in which, even for current steps greater than 3 times the rheobase, only one spike, always only at the onset of the stimulus, was fired (SSP; gray). (B) Number of cells per fast spiking subtype: continuous (dark blue), delayed (blue), and stuttering (cyan). 8 cells were recorded in PVAI32 animals, out of which 6 were fast spiking, one adapting and one non-adapting, non-fast spiking. The remaining was recorded in NKTDTO animals.

Eight out of 28 cells were recorded in PVAI32 animals and the remainder in NKTDTO animals. Six of the $8 \mathrm{PV}^{+}$cells (i.e., recorded in PVAI32 animals) were fast spiking neurons, while the other two were adapting and non-adapting non-fast spiking. As the e-type categorization is to a certain extend rather subjective, I decided to further characterize the action potential waveforms in each category, in order to find whether there are differences in the spike shape across e-types. Using an extensive database of interneuronal recordings, Druckman et al (2013) set out to identify by means of principal component analysis (PCA) which features, in a 38-dimension parameter space, are more informative to distinguish among e-types. They found that (1) action potential half-width, (2) afterhyperpolarization magnitude, and (3) rate of discharge are among the best parameters, particularly to distinguish between fast spiking and adapting interneurons. Figure 20 contains the values obtained for these parameters for CA, FS, NANFS, and SSP. 

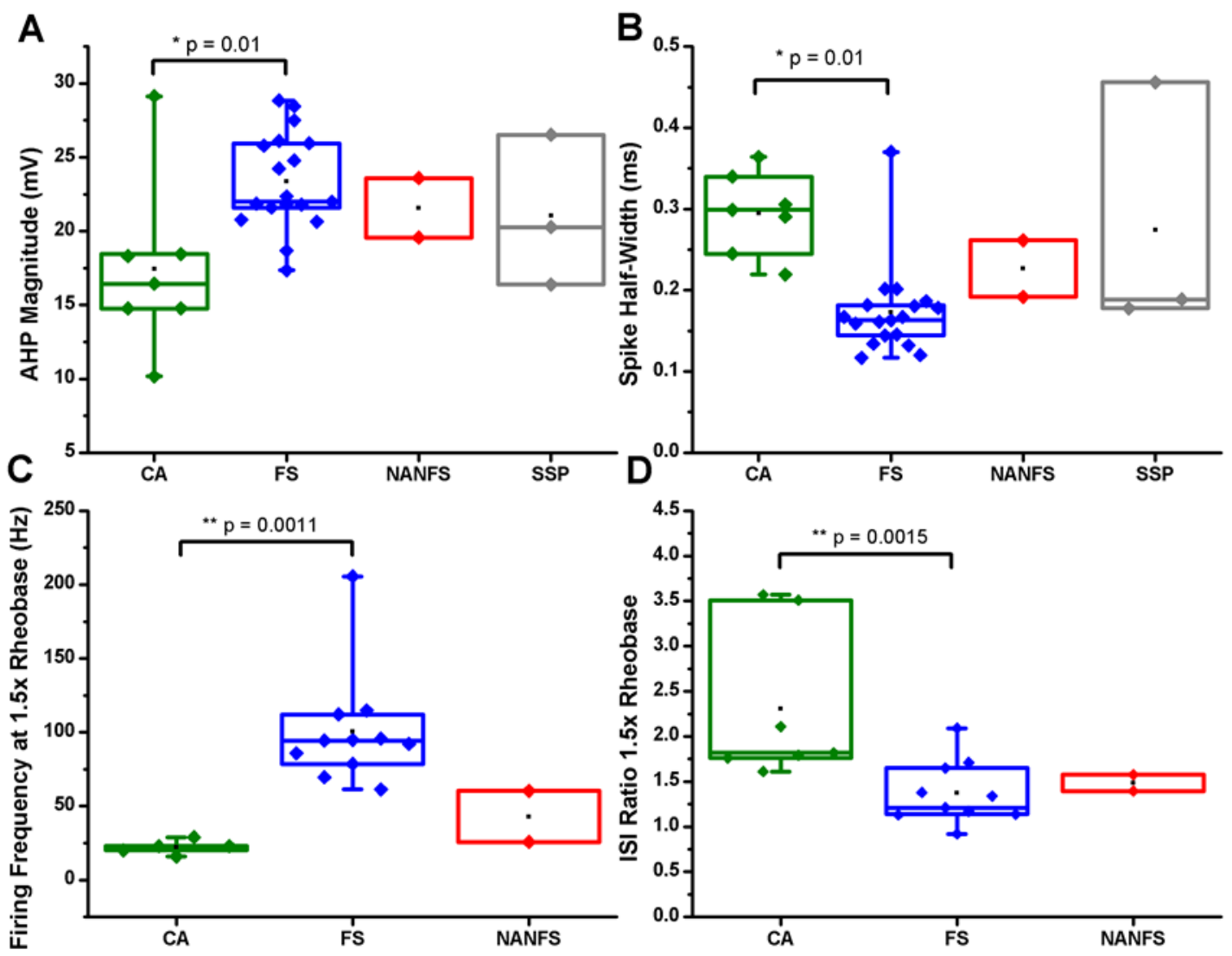

Figure 20. Differences in spike shape and firing frequency among e-types. (A) Afterhyperpolarization (AHP) magnitude. (B) Spike half-width. (C) Firing frequency at 1.5x rheobase. (D) Interspike interval (ISI) ratio, computed as the ratio of the last and first ISIs at $1.5 \mathrm{x}$ rheobase. Box-plots cover the whole range of data points, with $25 \%$ and $75 \%$ percentiles and median (horizontal line). Colored diamonds represent the average for each cell and the black square the overall average value. CA: continuous adapting; FS: fast spiking (continuous, delayed and stuttering, pooled together); NANFS: non-adapting, non-fast spiking. Statistical significance, as determined by a Mann-Whitney test between CA and FS etype only, is indicated by *, followed by the calculated $p$-value.

All of them were significantly different between the two most common e-types found (CA and FS neurons). In addition, the ratio between the last and first interspike intervals (ISI) at $1.5 \mathrm{x}$ rheobase level was also significant. As the number of NANFS and SSP neurons is low ( 2 and 3 cells, respectively), Due to a low number of samples, I did not perform statistical tests on these groups. Among the fast spiking subtypes, afterhyperpolarization magnitude and action potential half-width were similar (Figure 21). 

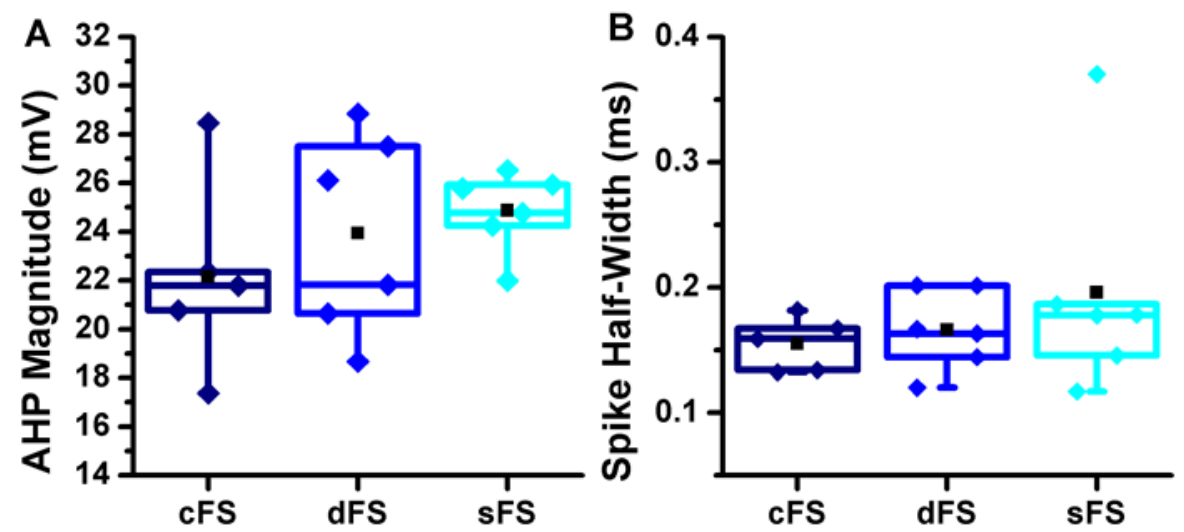

Figure 21. Comparison of afterhyperpolarization (AHP) amplitude (A) and spike halfwidth (B) between fast spiking cells. Box-plots cover the whole range of data points, with $25 \%$ and $75 \%$ percentiles and median (horizontal line). Colored diamonds represent the average for each cell and the black square the overall average value. cFS: continuous fast spiking; dFS: delayed fast spiking; sFS stuttering fast spiking.

Once the e-types were categorized, I set out to characterize the dynamic gain function of each subtype.

\subsubsection{Different electrical types encode information differently}

In order to determine the dynamic gain function of each interneuronal e-type, I used a paradigm similar to what has been traditionally used (Eyal et al., 2014; Higgs and Spain, 2009; Ilin et al., 2013; Tchumatchenko et al., 2011). Briefly, whole-cell current-clamp experiments were performed and a fluctuating current input emulating in-vivo activity (Destexhe et al., 2001; Destexhe et al., 2003) was somatically injected in neurons held at approximately $-60 \mathrm{mV}(-14 \mathrm{mV}$ liquid-junction potential not corrected). Input current standard deviation was adjusted so that a target firing rate range of $2-7 \mathrm{~Hz}$ was obtained. Two different noise-regimes were tested by changing the correlation time ( $\left.\tau_{\text {corr }}\right)$ of the fluctuating current: a "fast" regime, with $\tau_{\text {corr }}=5 \mathrm{~ms}$ (Figure 22) and a "slow" regime, with $\tau_{\text {corr }}=25 \mathrm{~ms}$ (Figure 23). Figures 22D and 23D contain the spike-triggered average (STA) calculated from multiple cells in each of these conditions. The fact that the STAs of different interneuronal e-types were different suggests that they encode information differently. In order to clarify this, I calculated the dynamic gain function of each e-type for both $\tau_{\text {corr. }}$. 
A

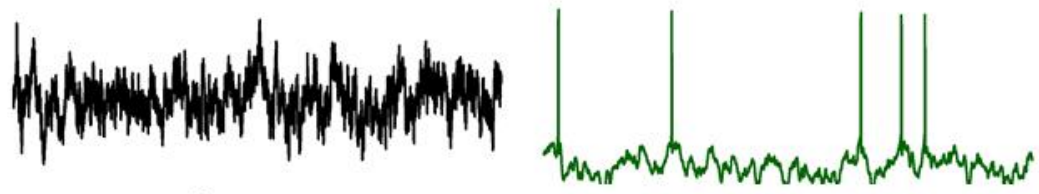

B
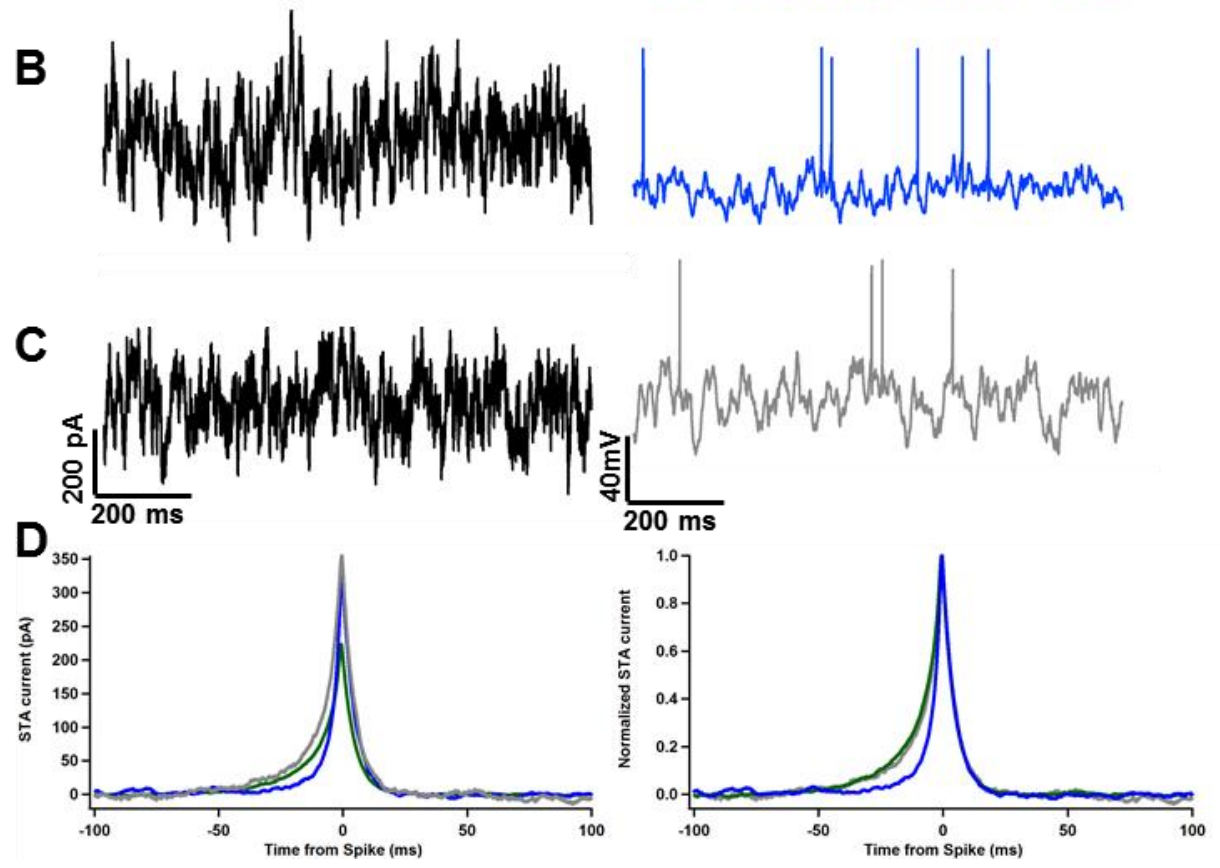

Figure 22. Examples of responses of different interneuron e-types to a $\tau_{\text {corr }}=\mathbf{5} \mathbf{m s}$ noise input (fast regime). A-C: Samples of the input current in the fast regime (left) and neuronal response (right) for (A) continuous adapting; (B) fast spiking; and (C) SSP. (D) Spike triggered average (STA) calculated from a total of 11582 spikes from 5 CA neurons (green), 8744 spikes from 9 FS cells (blue), and 2441 spikes from 3 SSP cells (left) and the normalized (with respect to the peak-value) STA (right). STAs were calculated by averaging $500 \mathrm{~ms}$ the input current centered at spike-times. For clarity, only the most significant part of the STAs was plotted. Average input current standard deviations were 95 pA, 127 pA, 166 pA for CA, FS and SSP respectively. Observe that the width of the STA of FS neurons is significantly narrower than for the other two e-types, indicating that signals of higher frequencies are better encoded by FS neurons.

Figure 24 shows the gain function of CA, FS and SSP neurons to 5-ms correlation time stimuli. Surprisingly, FS interneurons exhibited a greatly enhanced sensitivity to highfrequency components of the noise stimulus: a resonant peak can be seen starting at $30 \mathrm{~Hz}$ and peaking at approximately $200 \mathrm{~Hz}$. At around $400 \mathrm{~Hz}$ their response fell to values smaller than the gain at $1 \mathrm{~Hz}$. CA and SSP neurons exhibited a low-pass filter-like behavior, with greater gain in the 1 to $10 \mathrm{~Hz}$ range. Unfortunately none of the neurons tested in the fast fluctuation regime were NANFS cells. 
A

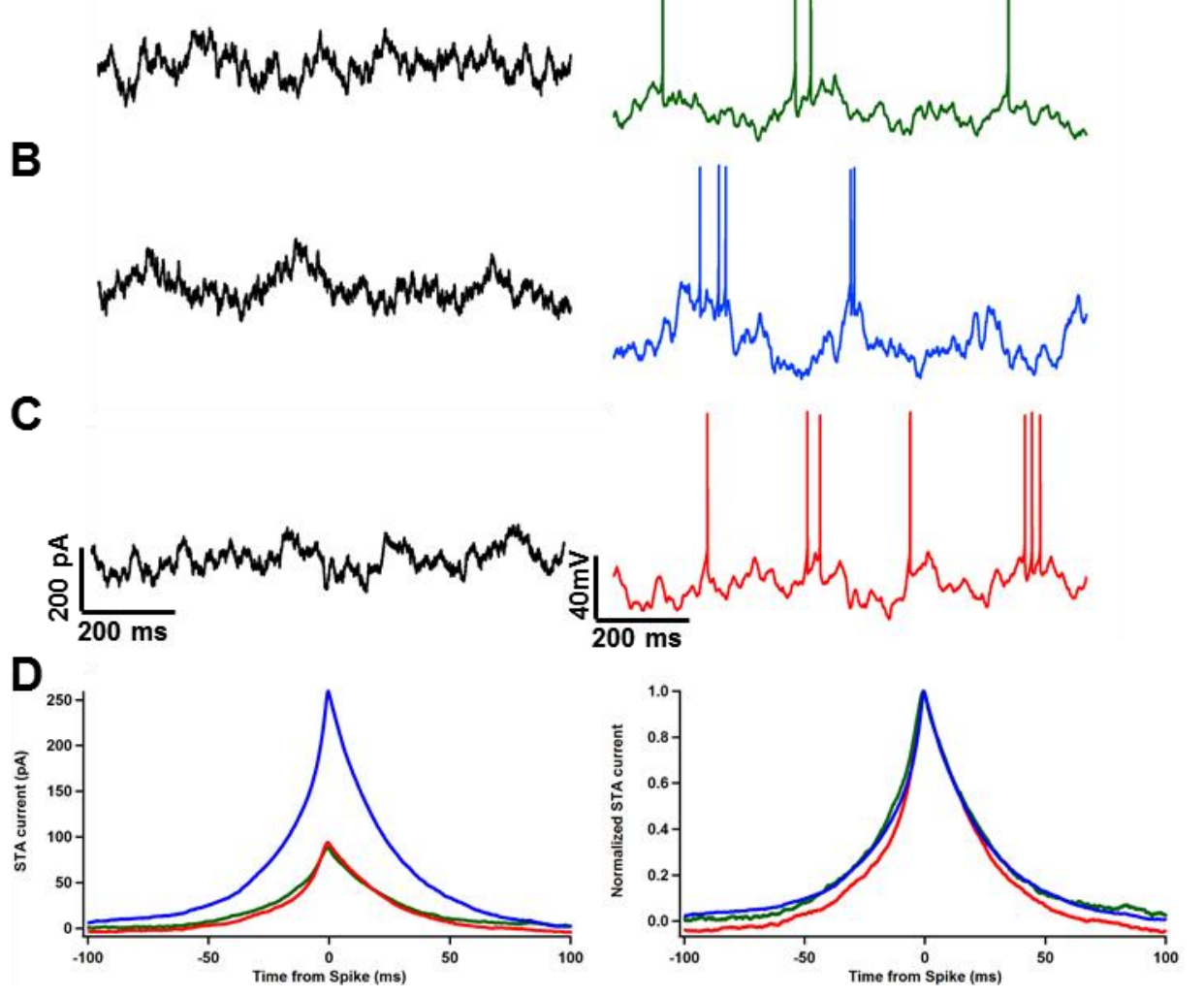

Figure 23.Examples of responses of different interneuron e-types to a $\tau_{\text {corr }}=25 \mathrm{~ms}$ noise input (slow regime). A-C: Samples of the input current in the slow regime (left) and neuronal response (right) for (A) continuous adapting; (B) fast spiking; and (C) non-adapting non-fast spiking cells. (D) Left: spike triggered average (STA) calculated from a total of 913 spikes from 2 CA neurons (green), 8042 spikes from 9 FS cells (blue), and 2803 spikes from 2 NANFS cells (red) and the normalized (with respect to the peak-value) STA (right). STAs were calculated by averaging $500 \mathrm{~ms}$ the input current centered at spike-times. For clarity, only the most significant part of the STAs was plotted. Average input current standard deviations were $43 \mathrm{pA}, 117 \mathrm{pA}, 50 \mathrm{pA}$ for CA, FS and NANFS respectively.

When tested in the slow noise regime, the gain functions changed to a much more homogeneous profile across the tested e-types (Figure 25). The big offset in the gain curves of CA and NANFS neurons vs FS neurons can be explained by the much larger input standard deviations used to drive FS neurons to the target firing frequency ( $43 \mathrm{pA}$ and 50 pA vs 117 $\mathrm{pA}$, respectively). When the offset is adjusted by normalizing the gains to the $1 \mathrm{~Hz}$ gain value (Figure 25B), one can observe that FS and CA gains, which were markedly different in the fast regime, are more alike at this regime, both exhibiting a band-pass like behavior, with FS neurons' gain, however, extending to higher frequencies (a comparison with the gain of the SSP neurons was not possible, since none of the cells tested in the slow regime were of this etype). 

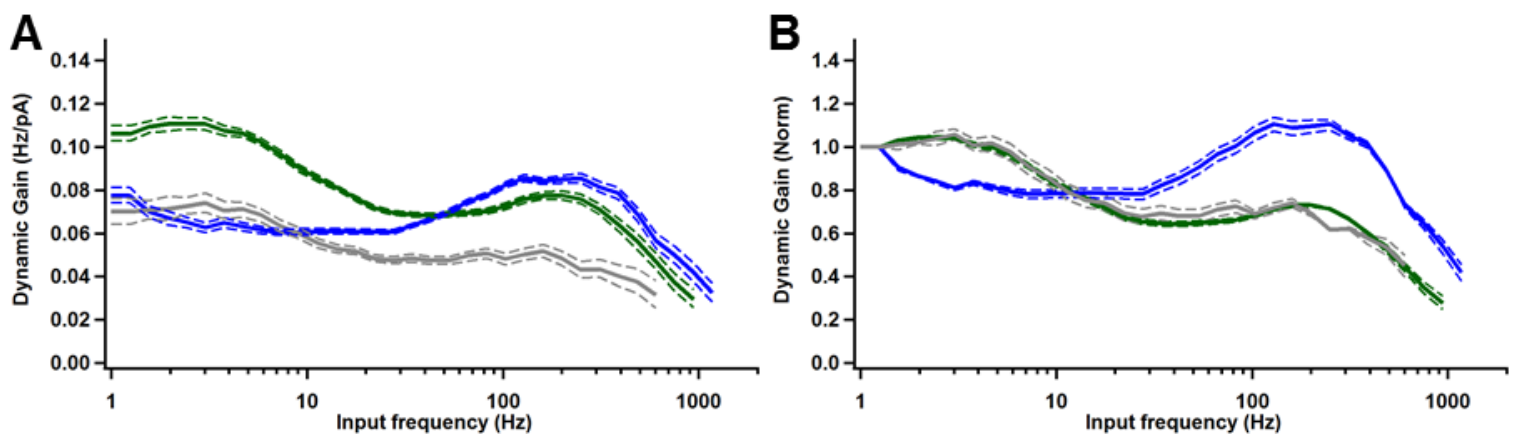

Figure 24. Dynamic gain function of interneuron e-types in the fast ( $\tau_{\text {corr }}=5 \mathbf{m s}$ ) noise regime. (A) Absolute gain function (solid line) for CA (green), FS (blue), and SSP (gray) cells. Dashed lines represent the $95 \%$ confidence interval of the gains. Gain functions were plotted until the frequency in which the lower-range confidence interval intersected with the calculated noise floor estimated from 200 gain curves obtained from random spike times (for clarity, not plotted here; see Methods for details). (B) Gain functions from (A) normalized by the gain at $1 \mathrm{~Hz}$. At this noise regime, FS neurons exhibited a resonance starting around $30 \mathrm{~Hz}$ and peaking at approximately $200 \mathrm{~Hz}$, while CA and SSP neurons were proportionally more sensitive to low-frequency components and behaved in a low-pass-filter fashion. Gains were calculated from the same data set as in figure $22 \mathrm{D}$.
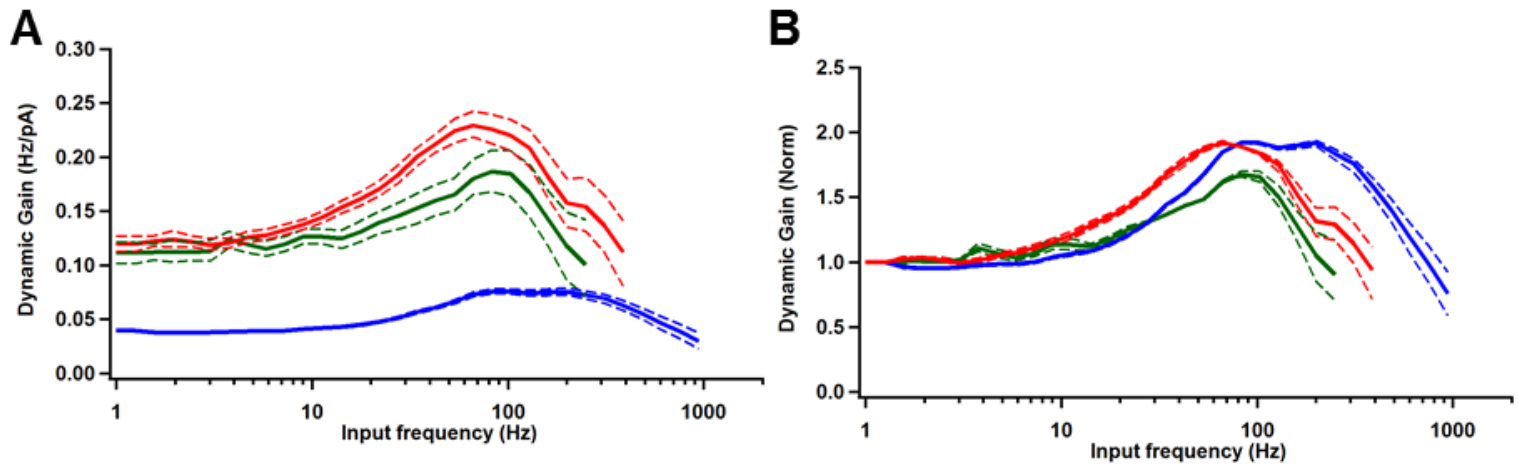

Figure 25. Dynamic gain function of interneuron e-types in the slow ( $\tau_{\text {corr }}=25 \mathrm{~ms}$ ) noise regime. (A) Absolute gain function for CA (green), FS (blue), and NANFS (red) cells. (B) Gain functions from (A) normalized by the gain at $1 \mathrm{~Hz}$. At slower regimes, FS, CA, and NANFS neurons all exhibited a band-pass behavior with peaking frequencies equal to 60,80 , and $80-200 \mathrm{~Hz}$ for NANFS, CA, and FS neurons respectively. In comparison to the other etypes, FS neurons were particularly more sensitive to frequencies above $200 \mathrm{~Hz}$ : remarkably, only at approximately $600 \mathrm{~Hz}$ their gains approaches the gain at the low-frequency range. Gains were calculated from the same data set as in figure $23 \mathrm{D}$.

In either case, FS neurons were clearly superior in coding high-frequency components, possibly due to the same biophysical mechanisms that allow these neurons to emit their typically fast action potentials at high frequencies. In the slow regime, while CA and NANFS gains fell below the $1 \mathrm{~Hz}$ value at approximately 190 and $300 \mathrm{~Hz}$, fast spiking interneurons were above the $1 \mathrm{~Hz}$-gain until approximately $620 \mathrm{~Hz}$, once more highlighting the increased sensitivity of these cells to high frequencies. For the two e-types in which the 
comparison between slow and fast regimes was possible, CA and FS, the gain in the highfrequency band was significantly higher at the slow noise regime, indicating that the so-called "Brunel effect" (Brunel et al., 2001; Tchumatchenko et al., 2011) found in pyramidal neurons is also present in interneurons. In order to better evaluate this phenomenon, figures $\mathbf{2 6 A}$ and B show the comparisons between the gains in the fast and slow regimes for CA and FS neurons, respectively. For both cells, the improvement in high-frequency band performance in the slow regime was clear, despite the fact that FS neurons already exhibited a highfrequency resonant peak in the fast regime.
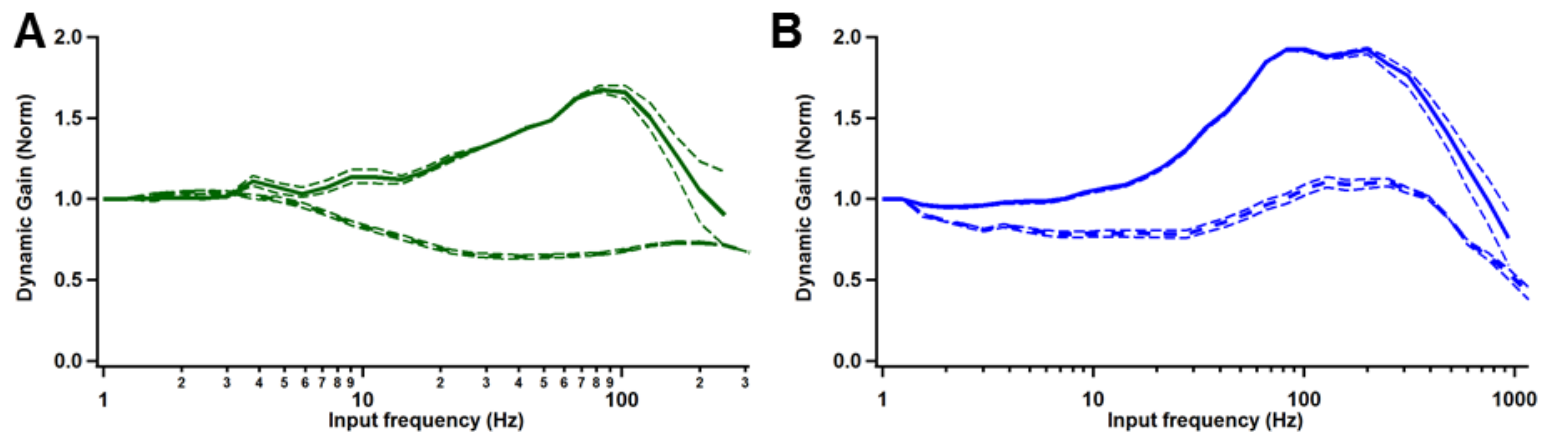

Figure 26. Comparison of the dynamic gain of CA and FS interneurons at the two different noise regimes. (A) Normalized gains of CA interneurons tested at the 5 ms (thick, dashed line), and $25 \mathrm{~ms}$ (thick, solid line) noise regimes. (B) Same as (A) for FS neurons. The gain in the high-frequency band is nearly doubled in the slow noise regime for both cells. Gains here depicted are the same from figures 22 and 23.

Theoretical and experimental evidences suggest that the action potential onset rapidness, i.e., the slope of the phase plot at the spike threshold value, is a major determinant of the high-frequency band of gain functions (Fourcaud-Trocme et al., 2003; Ilin et al., 2013; Naundorf et al., 2005; Wei and Wolf, 2011). For interneurons, however, this parameter did not seem to be determinant, since, despite the fact that the gain functions of CA and FS neurons are remarkably different, there was no significant statistical difference in the onset rapidness of these two neuronal e-types (Figure 27). Other parameters, such as action potential bursts might be responsible for the behavior seen in interneurons (Higgs and Spain, 2009). 

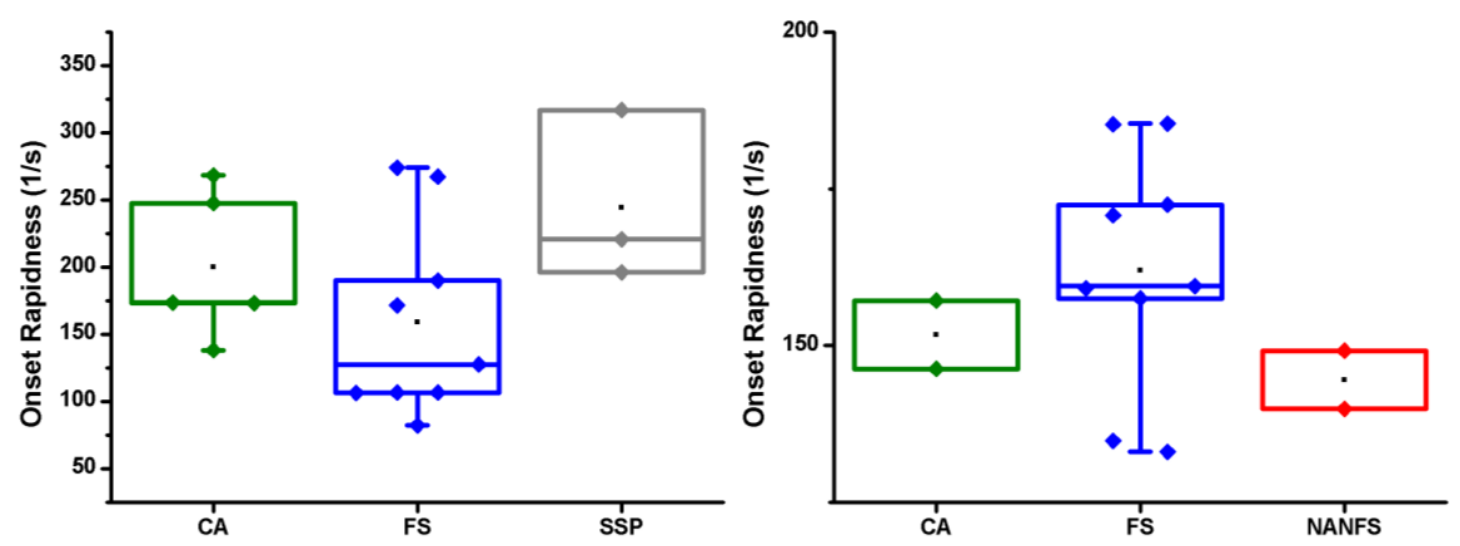

Figure 27. Onset rapidness for different interneuronal e-types at the fast and slow noise regimes. Onset rapidness is defined as the slope at the spike threshold level in the phase plot of the action potentials (defined as $30 \mathrm{~V} / \mathrm{s}$; see Methods). Each colored diamond represents the average for each cell and the black square represents the global average. Left: Fast noise regime. CA: continuous adapting, FS: fast spiking. Right: Slow noise regime. NANFS: non-adapting, non-fast spiking cells. No statistical significance was found between FS and CA neurons (Mann-Whitney test; $\mathrm{p}=0.254$ for $\mathrm{A}$; due to the low number of cells in the CA and NANFS groups $(n=2)$, no statistical test was performed for the slow noise regime).

\subsubsection{The gain curve of distinct fast spiking subtypes}

In the results shown above, continuous, delayed and stuttering fast spiking interneurons (cFS, dFS, and sFS, respectively) were considered a single group. But how do these different e-types of cells encode information? With the caveat that splitting the FS group in the three subgroups resulted in few cells per category ( 2 to 4 cells), which restricts the extension of the results, I performed the same analysis described above in each of the FS subtypes (Figures 28 and 29). At the $5 \mathrm{~ms}$ noise regime, the overall shape of the gain for the FS subtypes are similar. In general, all the gains exhibited a drop from $1 \mathrm{~Hz}$ until about $30 \mathrm{~Hz}$, when the values increase until reaching a peak in the 100 to $250 \mathrm{~Hz}$ range (Figure 28): cFS peaks at $130 \mathrm{~Hz}, \mathrm{sFS}$ at $250 \mathrm{~Hz}$, and dFS exhibits a peak plateau from 130 to $250 \mathrm{~Hz}$. 

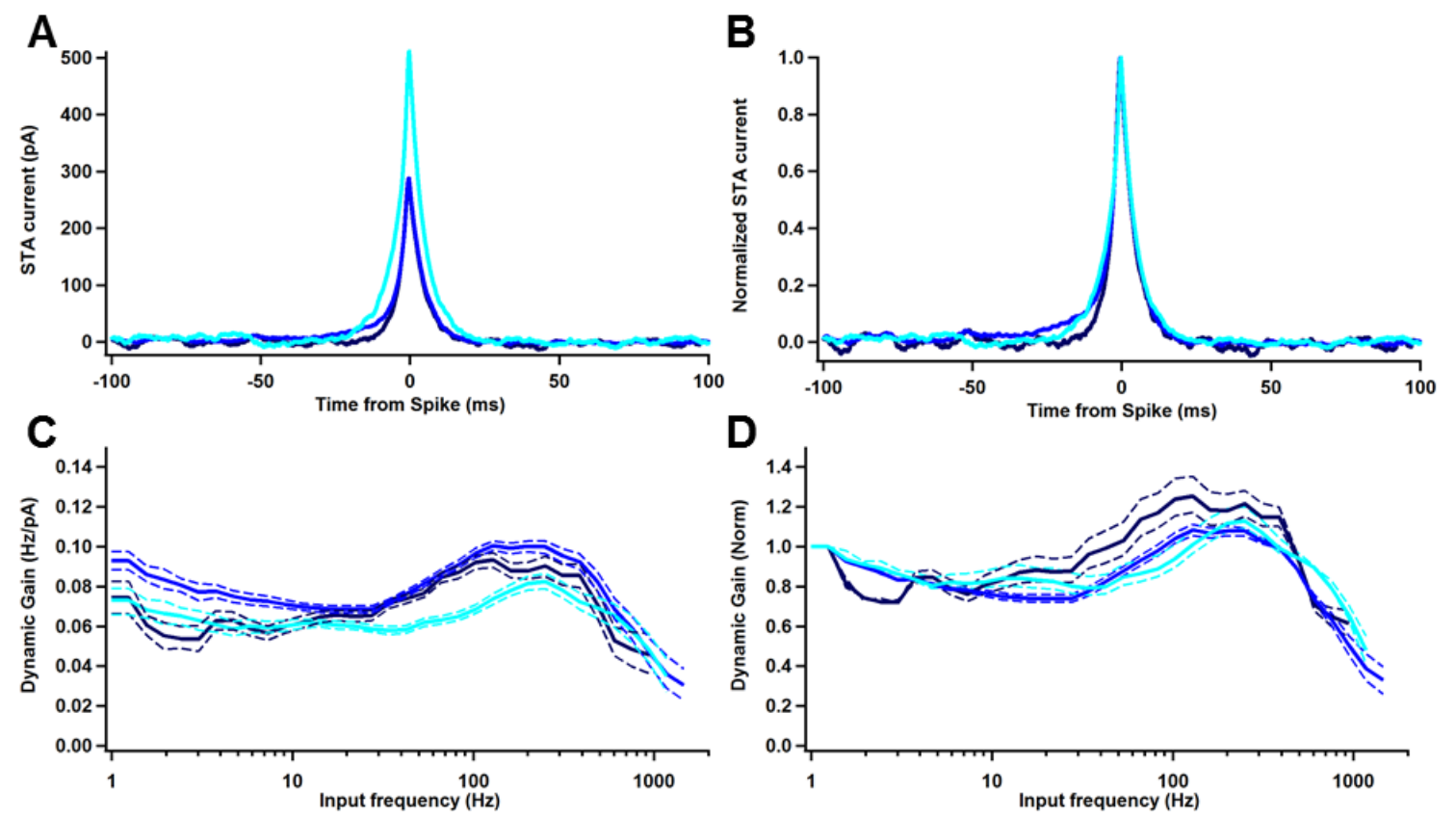

Figure 28. Gain differences in fast spiking subtypes in the fast noise regime $\left(\tau_{\text {corr }}=\mathbf{5}\right.$ ms). (A) Spike-triggered average (STA) from continuous (cFS; dark blue), delayed (dFS; blue), and stuttering (sFS; cyan) fast spiking neurons. 1580 spikes from 2 cells, 4867 spikes from 4 cells, and 2297 spikes from 3 cells were used to calculate the STAs of cFS, dFS, and sFS neurons respectively. (B) STAs from (A) normalized by their peak values. (C) Dynamic gain calculated from the STAs in (A). Gain peak values were $130 \mathrm{~Hz}$ and $250 \mathrm{~Hz}$ for cFS and sFS; dFS exhibited a high gain plateau in the $130-250 \mathrm{~Hz}$ range. (D) Gains normalized by the gain value at $1 \mathrm{~Hz}$. In (C) and (D), solid lines represent the average gain, while dashed lines the 95 $\%$ confidence interval of the respective gain. Gains were plotted until the frequency in which the lower confidence interval line intersected with the 95 percentile of 200 gains calculated from random spike times (not shown).

The cFS gain also displayed a sudden drop in at low frequencies. This was probably due to random fluctuations in the individual gain curve of one of the cells used to calculate the transfer function. As mentioned above, due to the small number of cells per conditions, gains were particularly susceptive to random fluctuations occurring, for example, due to changes in recording conditions. Regarding the slow noise regime, the gains were markedly different with respect to the maximum relative gain (Figure 29D): cFS and dFS gains were nearly constant in the 1 to $30 \mathrm{~Hz}$ range, from which cFS increased up to $40 \%$ at around $70 \mathrm{~Hz}$ and sFS increased $220 \%$, peaking at $160 \mathrm{~Hz}$, in comparison to the gain at $1 \mathrm{~Hz}$. The most significant increase in gain was in sFS cells, whose transfer function exhibited a $320 \%$ increase in the resonance peak at $200 \mathrm{~Hz}$. These results show that while qualitatively the gains of the fast spiking subtypes are similar, all exhibiting a band-pass-like behavior, stuttering cells are particularly sensitive to high-frequency components in the noise input. 

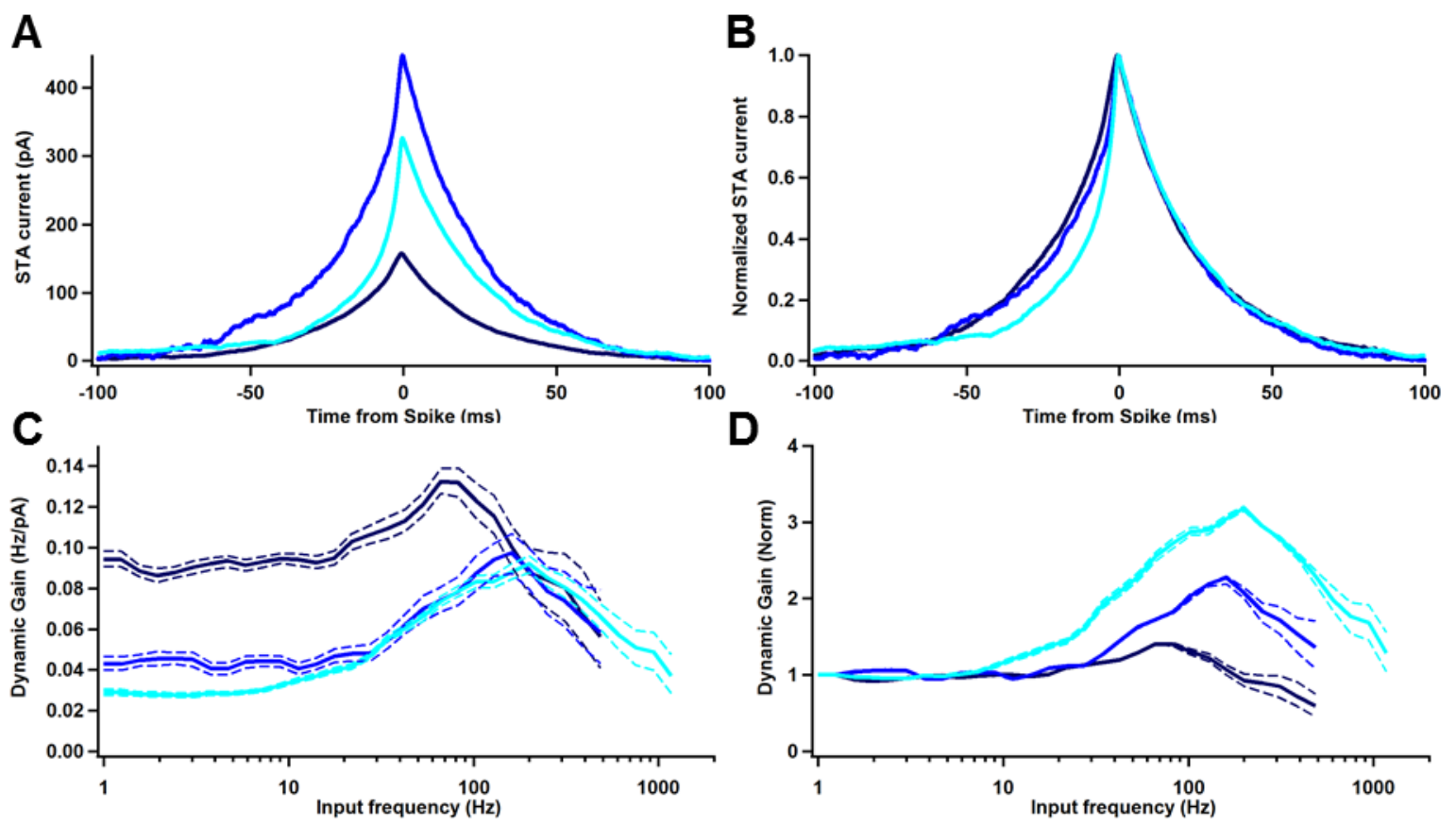

Figure 29. Gain differences in fast spiking subtypes in the slow noise regime ( $\tau_{\text {corr }}=\mathbf{2 5}$ ms). (A) Spike-triggered average (STA) from continuous (cFS; dark blue), delayed (dFS; blue), and stuttering (sFS; cyan) fast spiking neurons. 3237 spikes from 2 cells, 729 spikes from 2 cells, and 3694 spikes from 3 cells were used to calculate the STAs of cFS, dFS, and sFS neurons respectively. Overall, the two cFS cells required much smaller stimulus amplitudes to elicit spikes than cFS and sFS. (B) STAs from (A) normalized by their peak values. sFS STA was markedly different than cFS and dFS. (C) Dynamic gain calculated from the STAs in (A). The offset of $\mathrm{cFS}$ was due to the smaller input amplitude standard deviation required to induce firing in these cells. (D) Gains normalized by the gain value at $1 \mathrm{~Hz}$. In (C) and (D), solid lines represent the average gain, while dashed lines the $95 \%$ confidence interval of the respective gain. Gains were plotted until the frequency in which the lower confidence interval line intersected with the 95 percentile of 200 gains calculated from random spike times (not shown). 


\subsection{Discussion}

My results show for the first time the transfer function of subsets of interneurons, as defined by their electrical types. Essentially, I show that the classification of interneurons in different electrical types, based on the stereotypical responses they exhibit to depolarizing current steps, do result in subpopulations with distinct gain functions, which indicates that these e-types perform different input computations within their circuits, transmitting information of different spectral properties to their postsynaptic partners.

\subsubsection{What is the relationship between the electrical types here described and anatomically defined interneuron classes?}

An important question is: how are these e-types correlated with interneuronal classes? Interneurons form an extremely diverse class of neurons, and multimodal features, such as electrical behavior, morphology and neurochemical profiles, have been used to classify them (DeFelipe, 1993; Kawaguchi and Kubota, 1996, 1997). A major effort of the scientific community studying interneurons has aimed at the determination of the correlation between each of these modalities to each other (Ascoli et al., 2008; Druckmann et al., 2013; Kawaguchi and Kubota, 1997; Markram et al., 2004). With respect to the association between morphologically characterized interneurons and electrical types, multiple reports have shown that, often, the same interneuronal class exhibits more than one electrical type (Gupta et al., 2000; Markram et al., 2004; Nassar et al., 2015). However, certain patterns are common, such as the fast spiking behavior of parvalbulmin-positive ( $\left.\mathrm{PV}^{+}\right)$basket cells or the adapting firing behavior of somatostatin-positive Martinotti cells (Halabisky et al., 2006; Kawaguchi and Kubota, 1997; Nassar et al., 2015; Povysheva et al., 2008; Wang et al., 2004). In my work, the two major e-types found were exactly the fast spiking and the adapting, with most of the $\mathrm{PV}^{+}$cells (i.e., cells recorded from PVAI32 animals) being fast spikers (6 out of 8). The other 2 cells were adapting and non-adapting non-fast spiking, possibly belonging to a subpopulation of cells positive to both, parvalbulmin and somatostatin (Nassar et al., 2015). Together with basket cells, chandelier cells are also often included among the fast spiking cells (Jiang et al., 2015; Taniguchi et al., 2013). In the NKTDTO mouse line induced at late pregnancy, chandelier cells were shown to make up to $90 \%$ of the red-fluorescent proteinexpressing cells at layer II/III border in the medial prefrontal cortex (Taniguchi et al., 2013). However, due to the fact that recordings were also made in cells in frontal areas other than the medial prefrontal cortex (such as motor cortex), and layers other than II/III, it is not 
possible to claim that a significant fraction of the fast spiking cells I recorded were chandelier cells.

Nonetheless, as evident from the literature, an unequivocal classification of the recorded interneuron depends on more than correlational evidence from firing patterns or molecular markers. In order to clearly assign electrophysiological responses to a certain cell class, a morphological reconstruction of the recorded cell is necessary.

\subsubsection{What could be the main determinants of the different gain curves seen in different electrical types?}

To the best of my knowledge, this is the first time that gain functions for cells other than pyramidal neurons are calculated in the so-called noise-driven regime. This approach allows one to investigate the "preferred" input frequencies, i.e., those for which the cell's response is maximized. A fundamental difference between the gain functions I described with those of pyramidal neurons is of qualitative nature: pyramidal neurons typically exhibit a low-pass filter behavior in response to dynamic stimulation, with a cutoff frequency on the order of 200-400 Hz (Ilin et al., 2013; Kondgen et al., 2008; Tchumatchenko et al., 2011). In linear systems, this corresponds to response speeds on the submillisecond timescale (Tchumatchenko et al., 2011). The interneuronal gains I found strongly diverge from the typical low-pass filter behavior of pyramidal neurons, often exhibiting resonances at the highfrequency range. In addition, due to the resonant band, particularly in the slow noise regime, I showed that fast spiking cells are quantitatively different from pyramidal neurons, in which they are capable of following input at frequencies beyond the $600 \mathrm{~Hz}$ mark, indicating high precision firing capability. While factors such as membrane time-constant and maximum firing rate were thought to be the main determinants of the gain, the actual cutoff frequencies reached by pyramidal neurons is approximately 10 times higher than the expected from such parameters (Kondgen et al., 2008; Tchumatchenko et al., 2011). Theoretical models and experimental evidences in pyramidal neurons suggest that the action potential onset rapidness is the major responsible for neuronal cutoff frequency (Fourcaud-Trocme et al., 2003; Ilin et al., 2013; Naundorf et al., 2005; Tchumatchenko et al., 2011; Wei and Wolf, 2011). Ilin et al (2013) demonstrated both, experimentally and theoretically, that manipulations that reduce spike onset rapidness strongly impair the high-frequency gain of pyramidal neurons, as opposed to slowing down spikes by modifying $\mathrm{Na}^{2+}$ driving force or peak conductance, which only little changed the gain. In addition, Eyal et al (2014) have shown that impedance load imposed by dendritic trees of different sizes results in a 
reduction of the effective membrane time constant, which speeds up spike onset, thus increasing gain at higher frequencies as the dendritic tree increases.

In the fast noise regime, adapting (CA) and fast spiking (FS) neurons exhibited a fundamentally different gain behavior, with the first showing a maximum gain band at lower frequencies and the latter a strong resonance starting at $30 \mathrm{~Hz}$ and peaking at $120 \mathrm{~Hz}$. Nonadapting non fast spiking, and single spikers behaved fundamentally as adapting cells. Therefore all the discussion related to CA neurons applies likewise to these other two e-types. The significant qualitative differences on the gain behavior of CA and FS cells suggest that distinct factors might be the main gain determinants for these two e-types at this noise regime. Moreover, the fact that, despite exhibiting remarkably different gain functions, the onset rapidness among these two e-types is not significantly different, rules out this factor as a main determinant of the distinct gains I observed. Other factors, such as bursting activity, might also contribute to neuronal gain curves (Geisler et al., 2005; Higgs and Spain, 2009; Richardson et al., 2003). It has been shown that the gain curves of bursting neurons can exhibit resonance peaks, suggesting that these might be factors determinants of the gains of interneurons. Higgs and Spain (2009) showed that bursting layer II/II pyramidal neurons exhibit two resonance peaks, which are highly dependent on the statistics of the noise stimulus injected. In the case of a 5-ms exponentially-filtered stimulus, equivalent to the fast noise regime I used, they show that the pyramidal cell gain exhibits two peaks, centered at 8 $\mathrm{Hz}$ and $300 \mathrm{~Hz}$. Investigating the possible biophysical mechanisms that might cause such responses, Higgs and Spain (2009) show that the suppression of the fast afterdepolarizing current in these neurons both decreased bursting and lowered the gain at the resonance peaks. However, as pointed out by the authors themselves, a significant resonance persisted in the absence of bursts, suggesting that other biophysical mechanisms might be involved. Perisomatic voltage-dependent $\mathrm{K}^{+}$channels, found in fast spiking cells, have high threshold activation and fast deactivation, and have been shown to be fundamental for the emission of fast, repetitive spikes (Hu et al., 2014; Rudy and McBain, 2001). The presence of these channels might facilitate rapid firing of successive spikes, increasing the gain at certain frequencies.

The most distinctive feature between the two main e-types I found is the high spike adaptation seen in CA and absent in FS. Theoretical works have associate adapting currents to high-pass filtering of input (Benda and Herz, 2003), which is consistent with the slow regime gain behavior of these cells, but distinct from their behavior in the fast regime. Since it has been shown that dendritic load alters neuronal gain, and given that the dendritic arbor is highly variable among different interneurons, one can also speculate that, if the e-types do 
reflect distinct morphological classes, gain differences are to be expected. In a slice preparation, however, it is not trivial to infer about effects of dendritic trees on the gain given the fact that the slicing method can sever neurites in a differential and unpredictable way. A careful reconstruction of the recorded cells can also shed light onto dendritic filtering effects in the gain function. The fact that the different subtypes of fast spiking interneurons exhibited quantitatively marked differences, while their basic spike parameters were equivalent, indicates that aspects related to their firing patterns might be involved in gain determination. Furthermore, analysis on subthreshold gain and spike trains elicited by fluctuating stimulus is necessary in order to evaluate whether bursting activity and subthreshold membrane fluctuations can contribute to such differences.

\subsubsection{Functional implications}

At the fast noise regime, CA neurons exhibited higher gain at frequencies on the 1-8 $\mathrm{Hz}$ range, while $\mathrm{FS}$ gain increased in the $30-120 \mathrm{~Hz}$ range, plateauing until approximately 400 $\mathrm{Hz}$. These frequency ranges overlap, respectively with the theta (4-8 $\mathrm{Hz}$ ) and gamma oscillations (30-100 Hz) and with fast ripple oscillations (around $200 \mathrm{~Hz}$ ) (Buzsaki and Draguhn, 2004), indicating that these cells might be involved in these phenomena. In fact, it has been shown that optogenetic activation of FS neurons selectively amplifies gamma oscillations in-vivo (Cardin et al., 2009), and that somatostatin-containing hippocampal interneurons have membrane resonances at theta frequencies (Pike et al., 2000). While mainly found in the hippocampus, ripple oscillations have also been observed in the cortex of epileptic patients, where it may be associated to the initiation or propagation of seizures (Jirsch et al., 2006), and my results suggest that FS interneurons might be involved in these. With respect to CA neurons, theoretical and experimental work with adapting neurons have shown that spike adaptation regulates oscillations by inducing frequencies to which population firing becomes in phase with oscillations (Fuhrmann et al., 2002). Moreover, it has been shown that changes to these "zero-lag" frequencies induce respective changes in the phase-lag: slower inputs cause phase-advance, while faster inputs cause phase-lag (Fuhrmann et al., 2002). This behavior have been implicated in the emergence of population dynamics in recurrent networks (Csicsvari et al., 1999).

Being, to the best of my knowledge, the first of its type, my work indicates how different interneuronal electrical types encode information under two different noise regimes. The differences in the gains for different electrical types indicates that each of these types exhibit, in a noise correlation time-dependent manner, preferences with respect to input frequencies, and therefore are probably engaged in different network computations. In 
addition, the description of interneuronal gain function offers a valuable tool for theoretical works aiming at understanding how network dynamics emerge and are modulated. The biophysical mechanisms underlying, and setting the limits of, the gain in interneurons remains to be elucidated. 


\section{REFERENCES}

Adamantidis, A.R., Zhang, F., Aravanis, A.M., Deisseroth, K., and de Lecea, L. (2007). Neural substrates of awakening probed with optogenetic control of hypocretin neurons. Nature 450, 420-424.

Airan, R.D., Thompson, K.R., Fenno, L.E., Bernstein, H., and Deisseroth, K. (2009). Temporally precise in vivo control of intracellular signalling. Nature 458, 1025-1029.

Aravanis, A.M., Wang, L.P., Zhang, F., Meltzer, L.A., Mogri, M.Z., Schneider, M.B., and Deisseroth, K. (2007). An optical neural interface: in vivo control of rodent motor cortex with integrated fiberoptic and optogenetic technology. J Neural Eng 4, S143-156.

Ascoli, G.A., Alonso-Nanclares, L., Anderson, S.A., Barrionuevo, G., Benavides-Piccione, R., Burkhalter, A., Buzsaki, G., Cauli, B., Defelipe, J., Fairen, A., et al. (2008). Petilla terminology: nomenclature of features of GABAergic interneurons of the cerebral cortex. Nat Rev Neurosci 9, 557-568.

Atallah, B.V., Bruns, W., Carandini, M., and Scanziani, M. (2012). Parvalbumin-expressing interneurons linearly transform cortical responses to visual stimuli. Neuron 73, 159-170.

Bamann, C., Gueta, R., Kleinlogel, S., Nagel, G., and Bamberg, E. (2010). Structural guidance of the photocycle of channelrhodopsin-2 by an interhelical hydrogen bond. Biochemistry 49, 267-278.

Bamann, C., Kirsch, T., Nagel, G., and Bamberg, E. (2008). Spectral characteristics of the photocycle of channelrhodopsin-2 and its implication for channel function. J Mol Biol 375, 686694.

Battaglia, D., Karagiannis, A., Gallopin, T., Gutch, H.W., and Cauli, B. (2013). Beyond the frontiers of neuronal types. Front Neural Circuits 7, 13.

Benda, J., and Herz, A.V. (2003). A universal model for spike-frequency adaptation. Neural Comput $15,2523-2564$. 
Berndt, A., Prigge, M., Gradmann, D., and Hegemann, P. (2010). Two open states with progressive proton selectivities in the branched channelrhodopsin-2 photocycle. Biophys J 98, 753-761.

Berndt, A., Schoenenberger, P., Mattis, J., Tye, K.M., Deisseroth, K., Hegemann, P., and Oertner, T.G. (2011). High-efficiency channelrhodopsins for fast neuronal stimulation at low light levels. Proc Natl Acad Sci U S A 108, 7595-7600.

Berndt, A., Yizhar, O., Gunaydin, L.A., Hegemann, P., and Deisseroth, K. (2009). Bi-stable neural state switches. Nat Neurosci 12, 229-234.

Boyden, E.S., Zhang, F., Bamberg, E., Nagel, G., and Deisseroth, K. (2005). Millisecond-timescale, genetically targeted optical control of neural activity. Nat Neurosci 8, 1263-1268.

Brette, R. (2013). Sharpness of Spike Initiation in Neurons Explained by Compartmentalization. PLoS Comput Biol 9, e1003338.

Brunel, N., Chance, F.S., Fourcaud, N., and Abbott, L.F. (2001). Effects of synaptic noise and filtering on the frequency response of spiking neurons. Phys Rev Lett 86, 2186-2189.

Busskamp, V., Duebel, J., Balya, D., Fradot, M., Viney, T.J., Siegert, S., Groner, A.C., Cabuy, E., Forster, V., Seeliger, M., et al. (2010). Genetic reactivation of cone photoreceptors restores visual responses in retinitis pigmentosa. Science 329, 413-417.

Buzsaki, G., and Draguhn, A. (2004). Neuronal oscillations in cortical networks. Science 304, 19261929.

Buzsaki, G., and Wang, X.J. (2012). Mechanisms of gamma oscillations. Annu Rev Neurosci 35, 203-225.

Cardin, J.A., Carlen, M., Meletis, K., Knoblich, U., Zhang, F., Deisseroth, K., Tsai, L.H., and Moore, C.I. (2009). Driving fast-spiking cells induces gamma rhythm and controls sensory responses. Nature 459, 663-667.

Cauli, B., Zhou, X., Tricoire, L., Toussay, X., and Staiger, J.F. (2014). Revisiting enigmatic cortical calretinin-expressing interneurons. Front Neuroanat 8, 52. 
Csicsvari, J., Hirase, H., Czurko, A., Mamiya, A., and Buzsaki, G. (1999). Oscillatory coupling of hippocampal pyramidal cells and interneurons in the behaving Rat. J Neurosci 19, 274-287.

da Silva, M.P., Merino, R.M., Mecawi, A.S., Moraes, D.J., and Varanda, W.A. (2015). In vitro differentiation between oxytocin- and vasopressin-secreting magnocellular neurons requires more than one experimental criterion. Mol Cell Endocrinol 400, 102-111.

DeFelipe, J. (1993). Neocortical neuronal diversity: chemical heterogeneity revealed by colocalization studies of classic neurotransmitters, neuropeptides, calcium-binding proteins, and cell surface molecules. Cereb Cortex 3, 273-289.

DeFelipe, J., and Farinas, I. (1992). The pyramidal neuron of the cerebral cortex: morphological and chemical characteristics of the synaptic inputs. Prog Neurobiol 39, 563-607.

Deisseroth, K. (2011). Optogenetics. Nat Methods 8, 26-29.

Deisseroth, K. (2015). Optogenetics: 10 years of microbial opsins in neuroscience. Nat Neurosci $18,1213-1225$.

Destexhe, A., Rudolph, M., Fellous, J.M., and Sejnowski, T.J. (2001). Fluctuating synaptic conductances recreate in vivo-like activity in neocortical neurons. Neuroscience 107, 13-24.

Destexhe, A., Rudolph, M., and Pare, D. (2003). The high-conductance state of neocortical neurons in vivo. Nat Rev Neurosci 4, 739-751.

Druckmann, S., Hill, S., Schurmann, F., Markram, H., and Segev, I. (2013). A hierarchical structure of cortical interneuron electrical diversity revealed by automated statistical analysis. Cereb Cortex 23, 2994-3006.

Druga, R. (2009). Neocortical inhibitory system. Folia Biol (Praha) 55, 201-217.

Edelstein, A., Amodaj, N., Hoover, K., Vale, R., and Stuurman, N. (2010). Computer control of microscopes using microManager. Curr Protoc Mol Biol Chapter 14, Unit14 20. 
El Hady, A., Afshar, G., Broking, K., Schluter, O.M., Geisel, T., Stuhmer, W., and Wolf, F. (2013). Optogenetic stimulation effectively enhances intrinsically generated network synchrony. Front Neural Circuits 7, 167.

Eyal, G., Mansvelder, H.D., de Kock, C.P., and Segev, I. (2014). Dendrites impact the encoding capabilities of the axon. J Neurosci 34, 8063-8071.

Feil, R., Brocard, J., Mascrez, B., LeMeur, M., Metzger, D., and Chambon, P. (1996). Ligandactivated site-specific recombination in mice. Proc Natl Acad Sci U S A 93, 10887-10890.

Feldbauer, K., Zimmermann, D., Pintschovius, V., Spitz, J., Bamann, C., and Bamberg, E. (2009). Channelrhodopsin-2 is a leaky proton pump. Proc Natl Acad Sci U S A 106, 12317-12322.

Fourcaud-Trocme, N., and Brunel, N. (2005). Dynamics of the instantaneous firing rate in response to changes in input statistics. J Comput Neurosci 18, 311-321.

Fourcaud-Trocme, N., Hansel, D., van Vreeswijk, C., and Brunel, N. (2003). How spike generation mechanisms determine the neuronal response to fluctuating inputs. J Neurosci 23, 11628-11640.

Fuchs, E.C., Zivkovic, A.R., Cunningham, M.O., Middleton, S., Lebeau, F.E., Bannerman, D.M., Rozov, A., Whittington, M.A., Traub, R.D., Rawlins, J.N., et al. (2007). Recruitment of parvalbuminpositive interneurons determines hippocampal function and associated behavior. Neuron 53, 591604.

Fuhrmann, G., Markram, H., and Tsodyks, M. (2002). Spike frequency adaptation and neocortical rhythms. J Neurophysiol 88, 761-770.

Geisler, C., Brunel, N., and Wang, X.J. (2005). Contributions of intrinsic membrane dynamics to fast network oscillations with irregular neuronal discharges. J Neurophysiol 94, 4344-4361.

Govorunova, E.G., Sineshchekov, O.A., Janz, R., Liu, X., and Spudich, J.L. (2015). NEUROSCIENCE. Natural light-gated anion channels: A family of microbial rhodopsins for advanced optogenetics. Science 349, 647-650.

Gradinaru, V., Thompson, K.R., and Deisseroth, K. (2008). eNpHR: a Natronomonas halorhodopsin enhanced for optogenetic applications. Brain Cell Biol 36, 129-139. 
Gradinaru, V., Thompson, K.R., Zhang, F., Mogri, M., Kay, K., Schneider, M.B., and Deisseroth, K. (2007). Targeting and readout strategies for fast optical neural control in vitro and in vivo. J Neurosci 27, 14231-14238.

Gradinaru, V., Zhang, F., Ramakrishnan, C., Mattis, J., Prakash, R., Diester, I., Goshen, I., Thompson, K.R., and Deisseroth, K. (2010). Molecular and cellular approaches for diversifying and extending optogenetics. Cell 141, 154-165.

Gunaydin, L.A., Yizhar, O., Berndt, A., Sohal, V.S., Deisseroth, K., and Hegemann, P. (2010). Ultrafast optogenetic control. Nat Neurosci 13, 387-392.

Gupta, A., Wang, Y., and Markram, H. (2000). Organizing principles for a diversity of GABAergic interneurons and synapses in the neocortex. Science 287, 273-278.

Halabisky, B., Shen, F., Huguenard, J.R., and Prince, D.A. (2006). Electrophysiological classification of somatostatin-positive interneurons in mouse sensorimotor cortex. J Neurophysiol 96, 834-845.

Hegemann, P., Ehlenbeck, S., and Gradmann, D. (2005). Multiple photocycles of channelrhodopsin. Biophys J 89, 3911-3918.

Higgs, M.H., and Spain, W.J. (2009). Conditional bursting enhances resonant firing in neocortical layer 2-3 pyramidal neurons. J Neurosci 29, 1285-1299.

Hodgkin, A.L., and Huxley, A.F. (1952). A quantitative description of membrane current and its application to conduction and excitation in nerve. J Physiol 117, 500-544.

Hu, H., Gan, J., and Jonas, P. (2014). Interneurons. Fast-spiking, parvalbumin(+) GABAergic interneurons: from cellular design to microcircuit function. Science 345, 1255263.

Husson, S.J., Gottschalk, A., and Leifer, A.M. (2013). Optogenetic manipulation of neural activity in C. elegans: from synapse to circuits and behaviour. Biol Cell 105, 235-250.

Ilin, V., Malyshev, A., Wolf, F., and Volgushev, M. (2013). Fast computations in cortical ensembles require rapid initiation of action potentials. J Neurosci 33, 2281-2292. 
Jiang, X., Shen, S., Cadwell, C.R., Berens, P., Sinz, F., Ecker, A.S., Patel, S., and Tolias, A.S. (2015). Principles of connectivity among morphologically defined cell types in adult neocortex. Science 350 , aac9462.

Jirsch, J.D., Urrestarazu, E., LeVan, P., Olivier, A., Dubeau, F., and Gotman, J. (2006). Highfrequency oscillations during human focal seizures. Brain 129, 1593-1608.

Johansen, J.P., Hamanaka, H., Monfils, M.H., Behnia, R., Deisseroth, K., Blair, H.T., and LeDoux, J.E. (2010). Optical activation of lateral amygdala pyramidal cells instructs associative fear learning. Proc Natl Acad Sci U S A 107, 12692-12697.

Kato, H.E., Zhang, F., Yizhar, O., Ramakrishnan, C., Nishizawa, T., Hirata, K., Ito, J., Aita, Y., Tsukazaki, T., Hayashi, S., et al. (2012). Crystal structure of the channelrhodopsin light-gated cation channel. Nature 482, 369-374.

Kawaguchi, Y., and Kubota, Y. (1996). Physiological and morphological identification of somatostatin- or vasoactive intestinal polypeptide-containing cells among GABAergic cell subtypes in rat frontal cortex. J Neurosci 16, 2701-2715.

Kawaguchi, Y., and Kubota, Y. (1997). GABAergic cell subtypes and their synaptic connections in rat frontal cortex. Cereb Cortex 7, 476-486.

Klapoetke, N.C., Murata, Y., Kim, S.S., Pulver, S.R., Birdsey-Benson, A., Cho, Y.K., Morimoto, T.K., Chuong, A.S., Carpenter, E.J., Tian, Z., et al. (2014). Independent optical excitation of distinct neural populations. Nat Methods.

Kleinlogel, S., Feldbauer, K., Dempski, R.E., Fotis, H., Wood, P.G., Bamann, C., and Bamberg, E. (2011). Ultra light-sensitive and fast neuronal activation with the Ca2+-permeable channelrhodopsin CatCh. Nat Neurosci 14, 513-U152.

Kondgen, H., Geisler, C., Fusi, S., Wang, X.J., Luscher, H.R., and Giugliano, M. (2008). The dynamical response properties of neocortical neurons to temporally modulated noisy inputs in vitro. Cereb Cortex 18, 2086-2097.

Kubota, Y. (2014). Untangling GABAergic wiring in the cortical microcircuit. Curr Opin Neurobiol 26, 7-14. 
Lin, J.Y., Knutsen, P.M., Muller, A., Kleinfeld, D., and Tsien, R.Y. (2013). ReaChR: a red-shifted variant of channelrhodopsin enables deep transcranial optogenetic excitation. Nat Neurosci 16, 1499-1508.

Lin, J.Y., Lin, M.Z., Steinbach, P., and Tsien, R.Y. (2009). Characterization of engineered channelrhodopsin variants with improved properties and kinetics. Biophys J 96, 1803-1814.

Lopez-Munoz, F., Boya, J., and Alamo, C. (2006). Neuron theory, the cornerstone of neuroscience, on the centenary of the Nobel Prize award to Santiago Ramon y Cajal. Brain Res Bull 70, 391-405.

Lorenz-Fonfria, V.A., Resler, T., Krause, N., Nack, M., Gossing, M., Fischer von Mollard, G., Bamann, C., Bamberg, E., Schlesinger, R., and Heberle, J. (2013). Transient protonation changes in channelrhodopsin-2 and their relevance to channel gating. Proc Natl Acad Sci U S A 110, E12731281.

Madisen, L., Mao, T., Koch, H., Zhuo, J.M., Berenyi, A., Fujisawa, S., Hsu, Y.W., Garcia, A.J., 3rd, Gu, X., Zanella, S., et al. (2012). A toolbox of Cre-dependent optogenetic transgenic mice for lightinduced activation and silencing. Nat Neurosci 15, 793-802.

Madisen, L., Zwingman, T.A., Sunkin, S.M., Oh, S.W., Zariwala, H.A., Gu, H., Ng, L.L., Palmiter, R.D., Hawrylycz, M.J., Jones, A.R., et al. (2010). A robust and high-throughput Cre reporting and characterization system for the whole mouse brain. Nat Neurosci 13, 133-140.

Markram, H., Toledo-Rodriguez, M., Wang, Y., Gupta, A., Silberberg, G., and Wu, C. (2004). Interneurons of the neocortical inhibitory system. Nat Rev Neurosci 5, 793-807.

Mattis, J., Tye, K.M., Ferenczi, E.A., Ramakrishnan, C., O'Shea, D.J., Prakash, R., Gunaydin, L.A., Hyun, M., Fenno, L.E., Gradinaru, V., et al. (2012). Principles for applying optogenetic tools derived from direct comparative analysis of microbial opsins. Nat Methods 9, 159-172.

Metzger, D., Clifford, J., Chiba, H., and Chambon, P. (1995). Conditional site-specific recombination in mammalian cells using a ligand-dependent chimeric Cre recombinase. Proc Natl Acad Sci U S A 92, 6991-6995. 
Moyer, J.R., Jr., and Brown, T.H. (1998). Methods for whole-cell recording from visually preselected neurons of perirhinal cortex in brain slices from young and aging rats. J Neurosci Methods 86, 35-54.

Murphy, D. (1993). Caesarean section and fostering. Methods Mol Biol 18, 177-178.

Nagel, G., Brauner, M., Liewald, J.F., Adeishvili, N., Bamberg, E., and Gottschalk, A. (2005). Light activation of channelrhodopsin-2 in excitable cells of Caenorhabditis elegans triggers rapid behavioral responses. Curr Biol 15, 2279-2284.

Nagel, G., Ollig, D., Fuhrmann, M., Kateriya, S., Musti, A.M., Bamberg, E., and Hegemann, P. (2002). Channelrhodopsin-1: a light-gated proton channel in green algae. Science 296, 2395-2398.

Nagel, G., Szellas, T., Huhn, W., Kateriya, S., Adeishvili, N., Berthold, P., Ollig, D., Hegemann, P., and Bamberg, E. (2003). Channelrhodopsin-2, a directly light-gated cation-selective membrane channel. Proc Natl Acad Sci U S A 100, 13940-13945.

Namboodiri, V.M., and Stuber, G.D. (2016). Cell-Type-Specific Optogenetics in Monkeys. Cell 166, 1366-1368.

Nassar, M., Simonnet, J., Lofredi, R., Cohen, I., Savary, E., Yanagawa, Y., Miles, R., and Fricker, D. (2015). Diversity and overlap of parvalbumin and somatostatin expressing interneurons in mouse presubiculum. Front Neural Circuits 9, 20.

Naundorf, B., Geisel, T., and Wolf, F. (2005). Action potential onset dynamics and the response speed of neuronal populations. J Comput Neurosci 18, 297-309.

Naundorf, B., Wolf, F., and Volgushev, M. (2006). Unique features of action potential initiation in cortical neurons. Nature 440, 1060-1063.

Neef, A., El Hady, A., Nagpal, J., Bröking, K., Afshar, G., Schlüter, O.M., Geisel, T., Bamberg, E., Fleischmann, R., Stühmer, W., et al. (2013). Continuous Dynamic Photostimulation - inducing invivo-like fluctuating conductances with Channelrhodopsins. Preprint at arXiv arXiv:1305.7125.

Oesterhelt, D., and Stoeckenius, W. (1971). Rhodopsin-like protein from the purple membrane of Halobacterium halobium. Nat New Biol 233, 149-152. 
Olsen, S.R., Bortone, D.S., Adesnik, H., and Scanziani, M. (2012). Gain control by layer six in cortical circuits of vision. Nature 483, 47-52.

Pare, D., Shink, E., Gaudreau, H., Destexhe, A., and Lang, E.J. (1998). Impact of spontaneous synaptic activity on the resting properties of cat neocortical pyramidal neurons In vivo. J Neurophysiol 79, 1450-1460.

Pfeffer, C.K., Xue, M., He, M., Huang, Z.J., and Scanziani, M. (2013). Inhibition of inhibition in visual cortex: the logic of connections between molecularly distinct interneurons. Nat Neurosci.

Pi, H.J., Hangya, B., Kvitsiani, D., Sanders, J.I., Huang, Z.J., and Kepecs, A. (2013). Cortical interneurons that specialize in disinhibitory control. Nature.

Pike, F.G., Goddard, R.S., Suckling, J.M., Ganter, P., Kasthuri, N., and Paulsen, O. (2000). Distinct frequency preferences of different types of rat hippocampal neurones in response to oscillatory input currents. J Physiol 529 Pt 1, 205-213.

Povysheva, N.V., Zaitsev, A.V., Rotaru, D.C., Gonzalez-Burgos, G., Lewis, D.A., and Krimer, L.S. (2008). Parvalbumin-positive basket interneurons in monkey and rat prefrontal cortex. J Neurophysiol 100, 2348-2360.

Prigge, M., Schneider, F., Tsunoda, S.P., Shilyansky, C., Wietek, J., Deisseroth, K., and Hegemann, P. (2012). Color-tuned channelrhodopsins for multiwavelength optogenetics. J Biol Chem 287, 31804-31812.

Ramdya, P., Lichocki, P., Cruchet, S., Frisch, L., Tse, W., Floreano, D., and Benton, R. (2015). Mechanosensory interactions drive collective behaviour in Drosophila. Nature 519, 233-236.

Richardson, M.J., Brunel, N., and Hakim, V. (2003). From subthreshold to firing-rate resonance. J Neurophysiol 89, 2538-2554.

Ritter, E., Stehfest, K., Berndt, A., Hegemann, P., and Bartl, F.J. (2008). Monitoring light-induced structural changes of Channelrhodopsin-2 by UV-visible and Fourier transform infrared spectroscopy. J Biol Chem 283, 35033-35041. 
Roberts, T.F., Gobes, S.M., Murugan, M., Olveczky, B.P., and Mooney, R. (2012). Motor circuits are required to encode a sensory model for imitative learning. Nat Neurosci 15, 1454-1459.

Rudy, B., Fishell, G., Lee, S., and Hjerling-Leffler, J. (2011). Three groups of interneurons account for nearly $100 \%$ of neocortical GABAergic neurons. Dev Neurobiol 71, 45-61.

Rudy, B., and McBain, C.J. (2001). Kv3 channels: voltage-gated K+ channels designed for highfrequency repetitive firing. Trends Neurosci 24, 517-526.

Schneider, F., Gradmann, D., and Hegemann, P. (2013). Ion selectivity and competition in channelrhodopsins. Biophys J 105, 91-100.

Schneider, F., Grimm, C., and Hegemann, P. (2015). Biophysics of Channelrhodopsin. Annu Rev Biophys 44, 167-186.

Sherrington, C.S. (1906). The Integrative Action of the Nervous System. Yale University Press, New Haven.

Sineshchekov, O.A., Jung, K.H., and Spudich, J.L. (2002). Two rhodopsins mediate phototaxis to low- and high-intensity light in Chlamydomonas reinhardtii. Proc Natl Acad Sci U S A 99, 86898694.

Sohal, V.S., Zhang, F., Yizhar, O., and Deisseroth, K. (2009). Parvalbumin neurons and gamma rhythms enhance cortical circuit performance. Nature 459, 698-702.

Sussel, L., Marin, O., Kimura, S., and Rubenstein, J.L. (1999). Loss of Nkx2.1 homeobox gene function results in a ventral to dorsal molecular respecification within the basal telencephalon: evidence for a transformation of the pallidum into the striatum. Development 126, 3359-3370.

Taniguchi, H., He, M., Wu, P., Kim, S., Paik, R., Sugino, K., Kvitsiani, D., Fu, Y., Lu, J., Lin, Y., et al. (2011). A resource of Cre driver lines for genetic targeting of GABAergic neurons in cerebral cortex. Neuron 71, 995-1013.

Taniguchi, H., Lu, J., and Huang, Z.J. (2013). The spatial and temporal origin of chandelier cells in mouse neocortex. Science 339, 70-74. 
Tchumatchenko, T., Malyshev, A., Wolf, F., and Volgushev, M. (2011). Ultrafast population encoding by cortical neurons. J Neurosci 31, 12171-12179.

Thiele, T.R., Donovan, J.C., and Baier, H. (2014). Descending control of swim posture by a midbrain nucleus in zebrafish. Neuron $83,679-691$.

Thomson, A.M., and Lamy, C. (2007). Functional maps of neocortical local circuitry. Front Neurosci 1, 19-42.

Toettcher, J.E., Gong, D., Lim, W.A., and Weiner, O.D. (2011). Light-based feedback for controlling intracellular signaling dynamics. Nat Methods 8, 837-839.

Ullrich, S., Gueta, R., and Nagel, G. (2013). Degradation of channelopsin-2 in the absence of retinal and degradation resistance in certain mutants. Biol Chem 394, 271-280.

Verhoefen, M.K., Bamann, C., Blocher, R., Forster, U., Bamberg, E., and Wachtveitl, J. (2010). The photocycle of channelrhodopsin-2: ultrafast reaction dynamics and subsequent reaction steps. Chemphyschem 11, 3113-3122.

Wang, X., Zhang, C., Szabo, G., and Sun, Q.Q. (2013). Distribution of CaMKIllalpha expression in the brain in vivo, studied by CaMKIlalpha-GFP mice. Brain Res 1518, 9-25.

Wang, Y., Toledo-Rodriguez, M., Gupta, A., Wu, C., Silberberg, G., Luo, J., and Markram, H. (2004). Anatomical, physiological and molecular properties of Martinotti cells in the somatosensory cortex of the juvenile rat. J Physiol 561, 65-90.

Watakabe, A., Ohtsuka, M., Kinoshita, M., Takaji, M., Isa, K., Mizukami, H., Ozawa, K., Isa, T., and Yamamori, T. (2015). Comparative analyses of adeno-associated viral vector serotypes 1, 2, 5, 8 and 9 in marmoset, mouse and macaque cerebral cortex. Neurosci Res 93, 144-157.

Wefelmeyer, W., Cattaert, D., and Burrone, J. (2015). Activity-dependent mismatch between axoaxonic synapses and the axon initial segment controls neuronal output. Proc Natl Acad Sci U S A $112,9757-9762$.

Wei, W., and Wolf, F. (2011). Spike onset dynamics and response speed in neuronal populations. Phys Rev Lett 106, 088102. 
Williams, J.C., Xu, J., Lu, Z., Klimas, A., Chen, X., Ambrosi, C.M., Cohen, I.S., and Entcheva, E. (2013). Computational optogenetics: empirically-derived voltage- and light-sensitive channelrhodopsin-2 model. PLoS Comput Biol 9, e1003220.

Wilson, N.R., Runyan, C.A., Wang, F.L., and Sur, M. (2012). Division and subtraction by distinct cortical inhibitory networks in vivo. Nature 488, 343-348.

Woodruff, A.R., Anderson, S.A., and Yuste, R. (2010). The enigmatic function of chandelier cells. Front Neurosci 4, 201.

Woodruff, A.R., McGarry, L.M., Vogels, T.P., Inan, M., Anderson, S.A., and Yuste, R. (2011). Statedependent function of neocortical chandelier cells. J Neurosci 31, 17872-17886.

Zemelman, B.V., Lee, G.A., Ng, M., and Miesenbock, G. (2002). Selective photostimulation of genetically chARGed neurons. Neuron 33, 15-22. 


\section{LIST OF FIGURES}

Figure 1. Calculation of the dynamic gain function.

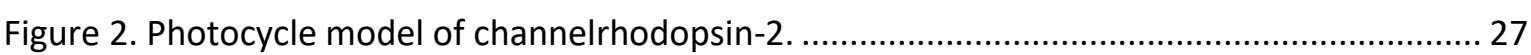

Figure 3. Different methods used to calculate neuronal gain function........................................ 32

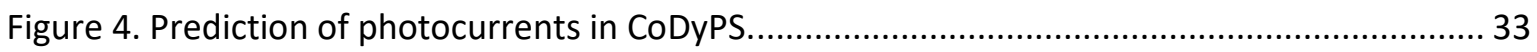

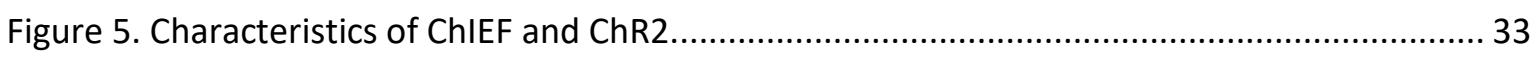

Figure 6. Example light-responses of the four light-sensitive channels tested. ...............................36

Figure 7. General properties of the photocurrents of the different light-sensitive channels tested.

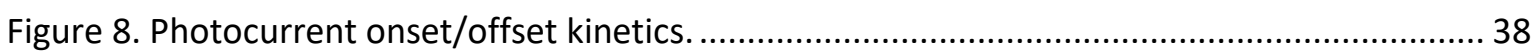

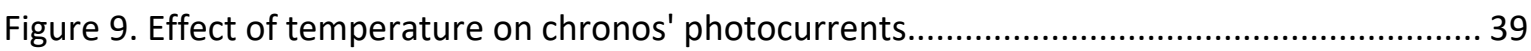

Figure 10. Quantification of activation (A) and deactivation (B) time-constants for chronos at

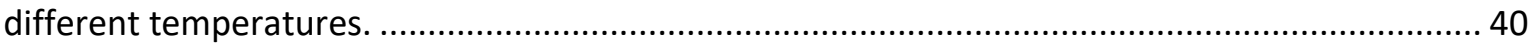

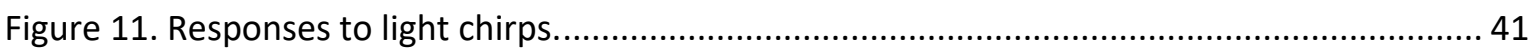

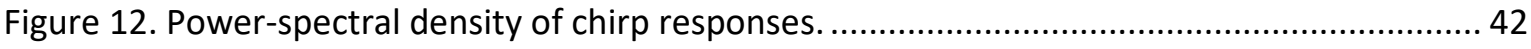

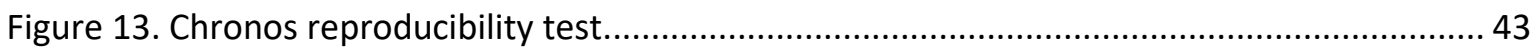

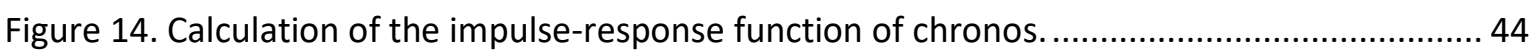

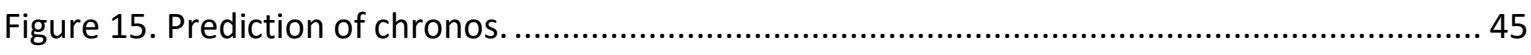

Figure 16. Sample photomicrographies of an AAV-CaMKII-eGFP in-utero injected animal. ............ 46

Figure 17. The classification of different interneuronal classes with respect to neurochemical

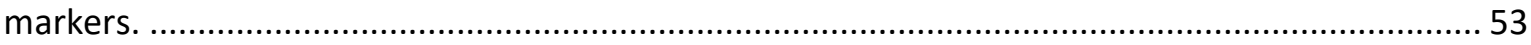

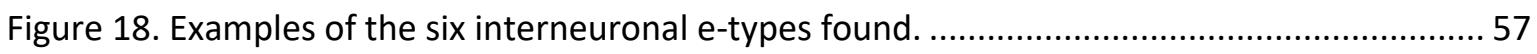

Figure 19. Number of cells per e-type (A) or per fast spiking subtype (B)................................... 58

Figure 20. Differences in spike shape and firing frequency among e-types.................................. 59

Figure 21. Comparison of afterhyperpolarization (AHP) amplitude (A) and spike half-width (B)

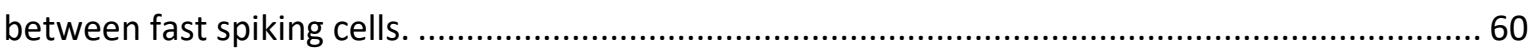

Figure 22. Examples of responses of different interneuron e-types to a $\tau_{\text {corr }}=5 \mathrm{~ms}$ noise input (fast

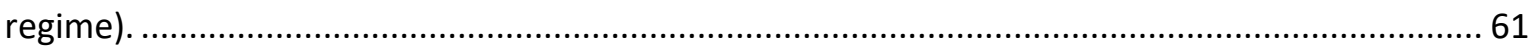

Figure 23.Examples of responses of different interneuron e-types to a $\tau_{\text {corr }}=25 \mathrm{~ms}$ noise input

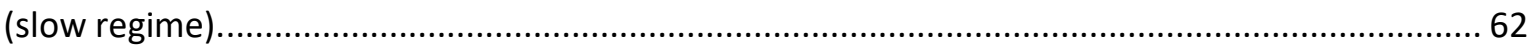

Figure 24. Dynamic gain function of interneuron e-types in the fast ( $\left.\tau_{\text {corr }}=5 \mathrm{~ms}\right)$ noise regime.... 63 Figure 25. Dynamic gain function of interneuron e-types in the slow $\left(\tau_{\text {corr }}=25 \mathrm{~ms}\right)$ noise regime. 63 Figure 26. Comparison of the dynamic gain of CA and FS interneurons at the two different noise regimes.

Figure 27. Onset rapidness for different interneuronal e-types at the fast and slow noise regimes.

Figure 28. Gain differences in fast spiking subtypes in the fast noise regime $\left(\tau_{\text {corr }}=5 \mathrm{~ms}\right)$...........66 66

Figure 29. Gain differences in fast spiking subtypes in the slow noise regime $\left(\tau_{\text {corr }}=25 \mathrm{~ms}\right)$........67 



\section{ACKNOWLEDGMENTS}

First and foremost, I would like to thank my supervisor, Prof. Dr. Fred Wolf, for allowing me to pursue my $\mathrm{PhD}$ in his lab, under his guidance. The experience of close interaction with theoreticians has definitely changed, in the most positive way, how I see and think science. The freedom I was given throughout my project has also strongly enriched to my personal and professional lives, and for this I thank Fred.

I cannot thank enough my co-supervisor, Dr. Andreas Neef, for all he has taught and helped me throughout the last four years. His patience, kindness, helpfulness and inexhaustible creativity for suggesting solutions to problems or new ideas have made my $\mathrm{PhD}$ experience so much smoother and pleasant.

I thank to Prof. Dr. Walter Stühmer for accepting me in his department and allowing me to take full advantage of all the resources I needed in his department. Being always supportive to me, and always with a nice suggestion to offer, he was also essential to the success of my $\mathrm{PhD}$ project.

Many aspects of my projects were only possible thank to resource donations from many contributors and collaborators. For that, I thank Prof Dr. Ernst Bamberg, Prof. Dr. Peter Hegemann, Prof. Dr. Karl Deisseroth, and Prof. Dr. Ed Boyden for donating the channelrhodopsin variants I used in my work; and I thank Prof. Dr. Tobias Moser, Prof. Dr. Klaus-Armin Nave, and Dr. Sonja Wojcik for donating some of the mouse lines I used in my experiments. Likewise, I would like to thank Prof. Dr. Luis Pardo for allowing me to share his equipment, reagents and for all the support when I needed.

From day-0 onwards, my experience in Göttingen has been wonderful. Much of this joy is directly connected to the amazing IMPRS-Neuroscience program of which I am proudly part of. Thank you so much for all people behind the program, and particularly to Prof. Dr. Michael Hörner and Sandra Drube for the absolute support they give to the students.

Thank you to the Göttingen Graduate School for Neurosciences, Biophysics, and Molecular Biosciences (GGNB) for making the graduate school experience such a high level and privileged experience and for also financially supporting me on my $\mathrm{PhD}$ by means of the Excellence Stipend I was awarded. 
Definitely a major contributor to this thesis is my beloved girlfriend Luísa. All her support, sweetness, love and help in these five years of Germany cannot be overstated. Love you so much, Fofinha! Thank you a lot!

My family that, even from far away, always supported me and my dreams; and my Göttingen family, Caio and Claudia, Eduardo and Thaís, Vinícius and Olga, Florian and Everlin, Lucas and Tati. Thank you all so much for being always there for me!

To my dearest friends from the lab, Adam Tomczak, Diana Urrego Blanco, and Carolina León Pinzón for all help in the projects, fun and for never giving up on me, despite my laziness and bad excuses for avoiding "some" social activities; Márcio Lazzarini and Fernanda Ramos Gomes, for helping me with questions, friendship, and for bringing a bit more of Brazil closer to me; Dennis Nestvogel for all lunch discussions throughout nearly 4 years and for his always excellent suggestions with project issues; Mohammad Khani for being my partner and motivator in our math sections. Jan Teer and Steffen Günzel for all the help with cell cultures and genotypings. Thank you all so much.

I would like to thank all the staff from the Department of Molecular Biology of Neuronal Signals, always very helpful and friendly, and in particular, Ute Rust for all her kindness and support offered whenever I needed. Thank you also to the supporting staff of the Max Planck Institute of Experimental Medicine, particularly the animal caretakers, for such an amazing work they do in the background. 


\title{
CURRICULUM VITAE
}

\author{
Ricardo Martins Merino, MSc
}

\section{PERSONAL DETAILS AND ADDRESS}

Address: $\quad$ Max Planck Institute for Dynamics and Self-Organization

Department of Nonlinear Dynamics

Phone: +49 (0)551 3899-620

Theoretical Neurophysics Group

E-mail: ricardo@nld.ds.mpg.de

Am Fassberg, 17

37077, Göttingen

Germany

\section{POST-SECONDARY EDUCATION}

10/2012-Present

PhD Student - Neurosciences Program - Göttingen Graduate School for Neurosciences, Biophysics, and Molecular Biosciences

Max Planck Institute for Dynamics and Self-Organization (MPI-ds) and Max Planck Institute of Experimental Medicine (MPI-em) - Göttingen Bernstein Center for Computational Neuroscience (BCCN) - Göttingen Supervisor: Prof. Dr. Fred Wolf (MPI-ds, BCCN-Göttingen)

Co-supervisors: Prof. Dr. Walter Stühmer (MPI-em, BCCN-Göttingen) Dr. Andreas Neef (MPI-ds, BCCN-Göttingen)

09/2011-09/2012 Student of the MSc-PhD International Max Planck Research School for Neurosciences, Göttingen.

02/2009-09/2011 Department of Physiology, Ribeirão Preto School of Medicine, University of São Paulo, Brazil

Supervisor: Prof. Dr. Wamberto Antônio Varanda

Degree: MSc (Physiology)

Thesis: Effects of angiotensin-(1-7) on the electrical activity of magnocellular neurons of the supraoptic nucleus of rats.

02/2004-08/2008 School of Philosophy, Sciences and Letters of Ribeirão Preto, University of São Paulo, Brazil

Supervisor: Prof. Dr. Ricardo Mauricio Xavier Leão

Degree: BSc (Biology)

Thesis: Immunofluorescence localization of non-muscular myosins in the calyx of Held synapse of rats 


\section{SCHOLARSHIPS}

- 12/2012 - 09/2016: Goettingen Graduate School for Neurosciences, Biophysics, and Molecular Biosciences Excellence Stipend

- 2011 - 2012: Stipend of the Excellence Foundation for the Promotion of the Max Planck Society

- 2009 - 2011: Scholarship from the Coordination for the Improvement of Higher Education Personnel, Brazilian Ministry of Education

- $\quad 2007$ - 2008: Scholarship from the National Council for Scientific and Technological Development, Brazilian Ministry of Science and Technology

\section{PUBLICATIONS}

- da Silva, M.P.*, R. M. Merino*, et al. (2015). "In Vitro differentiation between oxytocin- and vasopressin-secreting magnocellular neurons requires more than one experimental criterion." Molecular and cellular endocrinology 400: 102-111. (*authors contributed equally) 